\title{
Derivation of the Multimoment Hydrodynamics Equations for a Gas Mixture
}

\author{
Igor V. Lebed ${ }^{1}$ \\ ${ }^{1}$ Zhukovsky Central Institute of Aerohydrodynamics, Moscow, Russia \\ Correspondence: Igor V. Lebed, Zhukovsky Central Institute of Aerohydrodynamics, Moscow, Russia. \\ E-mail:lebed-ivl@yandex.ru
}

Received: May 17, 2016

Accepted: June 9, 2016

Online Published: July 29, 2016

doi:10.5539/apr.v8n4p103

URL: http://dx.doi.org/10.5539/apr.v8n4p103

\begin{abstract}
The equations for pair distribution functions are used to derive the multimoment hydrodynamics equations for gas mixture. The gas mixture pair distribution functions are specified. The equations for pair functions are derived directly from the general statistical mechanics concepts. The basic property of the pair functions is established. In conformity with basic property, these functions remain unchanged in time along the trajectory of the center of inertia of a pair. The basic property of the pair distribution functions reveals the existence of an infinite number of principle hydrodynamic values. Multimoment hydrodynamics equations are constructed using $3 L+4$ principle hydrodynamic values, where $L$ is the number of gas mixture components. Just these principle values specify measurable moments. The measurable moments are represented by linear combination of principle and non-principle hydrodynamic values. The general structure of constructed multimoment conservation laws is identical to the structure of appropriate multimoment conservation laws in a gas of identical particles. Each of the multimoment conservation laws is divided into two separate equations. The first group of conservation equations corresponds to convective phenomena. The second group of conservation equations corresponds to dissipative phenomena. Derived multimoment hydrodynamics equations are designed for interpreting the behavior of unstable systems. As is shown previously, classic hydrodynamics equations are incapable of reproducing flows after they lose stability. That is, the solutions to the classic hydrodynamics equations do not find the direction of instability development correctly. The possibility of improvement of classic hydrodynamics equations for a gas mixture is sought on the way toward an increase in the number of principle hydrodynamic values.
\end{abstract}

Keywords: multimoment hydrodynamics, pair distribution functions, instability

\section{Introduction}

The results of the direct numerical integration of the Navier-Stokes equations were evaluated against experimental data for problem of a flow around a bluff body in unstable regime (Lebed \& Umanskii, 2007; Lebed, 2013). It was revealed that calculations satisfactory reproduce all stable medium states observed experimentally. However, calculations are incapable of producing anything that corresponds to numerous unstable regimes observed along several directions of instability development.

The problems encountered by classic hydrodynamics when interpreting the unstable phenomena were not unexpected. They were predicted previously. Classic kinetics and classic hydrodynamics are direct corollaries to the first equation of the BBGKY hierarchy. The Boltzmann hypothesis of molecular chaos "Stosszahlansatz" closes kinetic equation for one-particle distribution function (Liboff, 1969). The classic hydrodynamics equations follow directly from the Boltzmann equation and, quite naturally, involve the error inherent in the derivation of the classic kinetic equation. The physical meaning of the error introduced by the Boltzmann hypothesis into hydrodynamics was disclosed by Lebed $(1995,2013)$. Analysis of the properties of collision integral (Lebed, 1996a; Lebed \& Umanskii, 2012) reveals the existence of an infinite number of principle hydrodynamic values. It was shown that just the Boltzmann hypothesis allowed us to construct the classic hydrodynamics equations for only three lower principle hydrodynamic values. It follows that the use of the Boltzmann hypothesis excludes higher principle hydrodynamic values from the participation in the formation of classic hydrodynamics equations. 
The possibility of improvement of classic hydrodynamics equations was found by Lebed (1990) where equations for pair distribution functions were derived heuristically. The equations for pair distribution functions are direct corollaries to the second equation of the BBGKY hierarchy. The multimoment hydrodynamics equations follow directly from the equations for pair distributions functions. The multimoment hydrodynamics equations were derived for the rarefied gas of identical structureless particles by Lebed (1996a). The formalism of Lebed (1996a)allows hydrodynamics equations to be derived with an arbitrary number of principle hydrodynamic values specified beforehand. The multimoment hydrodynamics equations are constructed using seven principal hydrodynamic values by Lebed (1996a). Just these seven principal hydrodynamic values specify measurable moments determined by the one-particle distribution function.

The multimoment hydrodynamics equations were used to study the phenomenon of instability appearance and development in the problem of flow around a solid sphere at a wide range of Reynolds number values (Lebed, 1997; 1998; 2014a; 2014b). It was found that solutions to these equations lead the development of instability in the direction compatible with experiment.

It was discovered that stability loss is accompanied by the appearance of uncommon acts at scenario of instability development. They are the disintegration of the Gibbs ensemble (Lebed, 1997; 2014b), the breakage of unstable solution (Lebed, 1997; 2014b), the movement of a system at an extremely unlikely direction described by the reverse multimoment hydrodynamics equations (Lebed, 2014b; 2014c; 2015b).It was discovered that system entropy begins to play the key role to predict the direction of instability development. The calculation of the system evolution becomes impossible without participation of the entropy. The multimoment hydrodynamics equations find a large number of ways for system evolution. Just the entropy shows the single way, in which the system evolution goes (Lebed, 2014b; 2015b).

The present work is devoted to the derivation of the multimoment hydrodynamics equations for the gas mixture of structureless particles. The original equations are represented in Section 2. Relations between two-particle and pair, and paired-particles distribution functions are written in Section 3. Equations represented in Section 2 are used to derive equations for pair and paired-particles distribution functions in Section 4. In Section 5, the moments of one-particle distribution function are expressed in terms of pair distribution functions. In Section 6, pair distribution functions are expended as a Grad series. Equations represented in Section 4 are invoked to derive the multimoment hydrodynamics equations in Section 7. The results of derivation are discussed in Section 8.

\section{Original Equations}

Suppose that some physical system consists of $N_{1}$ structureless particles of quality $1, N_{2}$ structureless particles of quality $2, \ldots$, and $N_{L}$ structureless particles of quality $L, N_{1}+N_{2}+\ldots+N_{L}=N$. Let us describe the physical system at the statistical deterministic level of description. The $N$-particle distribution function contains statistical information about all $N$ particles of the system. This information is overly detailed. The reduction of the ordinal number of particle distribution function accompanies by the loss of information about the position in phase space of the particle in turn. The $N$-particle distribution function obeys the Liouville equation (Liboff, 1969). The two-particle distribution function $F_{2(i, j)}\left(t, \mathbf{x}_{1}, \boldsymbol{\xi}_{1}, \mathbf{x}_{2}, \boldsymbol{\xi}_{2}\right)$ obeys the second equation of the BBGKY hierarchy for a gas mixture,

$$
\begin{aligned}
& \left(\frac{\partial}{\partial t}+\xi_{1} \frac{\partial}{\partial \mathbf{x}_{1}}+\xi_{2} \frac{\partial}{\partial \mathbf{x}_{2}}+\frac{\boldsymbol{\Phi}_{1,2}}{m_{i}} \frac{\partial}{\partial \xi_{1}}+\frac{\boldsymbol{\Phi}_{2,1}}{m_{j}} \frac{\partial}{\partial \xi_{2}}\right) F_{2(i, j)}\left(t, \mathbf{x}_{1}, \boldsymbol{\xi}_{1}, \mathbf{x}_{2}, \boldsymbol{\xi}_{2}\right)+ \\
& +\left(N_{i}-1\right) \int\left(\frac{\boldsymbol{\Phi}_{1,3}}{m_{i}} \frac{\partial}{\partial \xi_{1}}+\frac{\boldsymbol{\Phi}_{2,3}}{m_{j}} \frac{\partial}{\partial \xi_{2}}\right) F_{3(i, j, i)}\left(t, \mathbf{x}_{1}, \xi_{1}, \mathbf{x}_{2}, \xi_{2}, \mathbf{x}_{3}, \xi_{3}\right) d \mathbf{x}_{3} d \xi_{3}+ \\
& +\left(N_{j}-1\right) \int\left(\frac{\boldsymbol{\Phi}_{1,3}}{m_{i}} \frac{\partial}{\partial \xi_{1}}+\frac{\boldsymbol{\Phi}_{2,3}}{m_{j}} \frac{\partial}{\partial \xi_{2}}\right) F_{3(i, j, j)}\left(t, \mathbf{x}_{1}, \xi_{1}, \mathbf{x}_{2}, \xi_{2}, \mathbf{x}_{3}, \boldsymbol{\xi}_{3}\right) d \mathbf{x}_{3} d \xi_{3}+ \\
& +\sum_{k=1, k \neq i, k \neq j}^{L} N_{k} \int\left(\frac{\boldsymbol{\Phi}_{1,3}}{m_{i}} \frac{\partial}{\partial \xi_{1}}+\frac{\boldsymbol{\Phi}_{2,3}}{m_{j}} \frac{\partial}{\partial \xi_{2}}\right) F_{3(i, j, k)}\left(t, \mathbf{x}_{1}, \boldsymbol{\xi}_{1}, \mathbf{x}_{2}, \boldsymbol{\xi}_{2}, \mathbf{x}_{3}, \boldsymbol{\xi}_{3}\right) d \mathbf{x}_{3} d \boldsymbol{\xi}_{3}=0
\end{aligned}
$$


In Equation (2.1), $m_{i}$ is the mass of the particle of $i$-quality, $\boldsymbol{\Phi}_{i, j}$ is the force of action of the particle of $i$-quality on the particle of $j$-quality. The two-particle distribution function $F_{2(i, j)}\left(t, \mathbf{x}_{1}, \boldsymbol{\xi}_{1}, \mathbf{x}_{2}, \boldsymbol{\xi}_{2}\right)$ has a meaning of the probability that at some time $t$ one particle of $i$-quality, say particle 1 , finds itself within unit element of phase space near point $\mathbf{x}_{1}, \xi_{1}$, and another particle of $j$-quality, say particle 2 , within unit element near point $\mathbf{x}_{2}, \xi_{2}$, regardless of the position in phase space of the remaining $N-2$ particles. In the three-particle distribution function $F_{3(i, j, l)}\left(t, \mathbf{x}_{1}, \boldsymbol{\xi}_{1}, \mathbf{x}_{2}, \boldsymbol{\xi}_{2}, \mathbf{x}_{3}, \boldsymbol{\xi}_{3}\right)$, the third particle of $l$-quality, $l=1, \ldots, L$, finds itself within unit element of phase space near point $\mathbf{x}_{3}, \xi_{3}$. The distribution function $F_{2(i, j)}\left(t, \mathbf{x}_{1}, \xi_{1}, \mathbf{x}_{2}, \boldsymbol{\xi}_{2}\right)$, $i \neq j$, is non-invariant with respect to permutation of pairs of arguments $\mathbf{x}_{1}, \boldsymbol{\xi}_{1}$, and $\mathbf{x}_{2}, \boldsymbol{\xi}_{2}$. In the thermodynamic limit, $N_{i} \rightarrow \infty, V \rightarrow \infty$, yet $N / V$ is a finite, $i=1, \ldots, L, V$ is the volume of the system.

The solutions to the equation (2.1) describe the system at the finest initial stage as well as at the kinetic and hydrodynamic stages (Liboff, 1969). Passage to less detailed kinetic description stage is implemented by neglecting the information about a sharp change of the distribution functions on the initial $l_{0}$-scale. Namely, the distribution function governed the equations of kinetic description stage, varies strongly on kinetic $l_{k}-$ scale After transition to the most coarse hydrodynamic description stage the distribution function varies strongly on hydrodynamic $l_{h}$-scale only. The spatial interval $l_{0}$ is identical to the characteristic radius of interparticle interaction potential $d$. The spatial interval $l_{k}$ is identical to the characteristic free path length $\lambda$. The spatial interval $l_{h}$ is equivalent to the characteristic spatial scale of flow $L_{h}$.

In accordance with observations formed the ideas of the kinetic theory of gases on a free path, in rarefied gas at each time moment, each particle after its last collision moves toward the next collision. This means that every particle 1 in a rarefied gas simultaneously flies away from some particle 2 with which it collided last at point $D$ (Figure 1a) and approaches some particle 2 with which it is to collide next at point $B$ (Figure 1b).

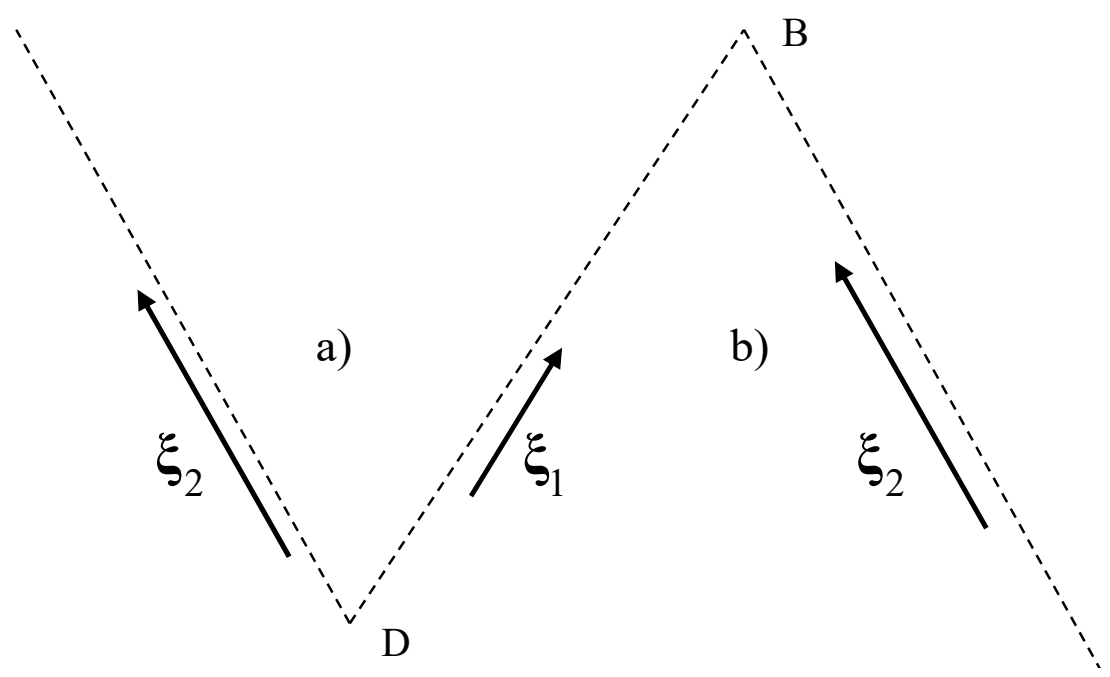

Figure 1. Graphic representation of a pair of particles.

Let particles 1 and 2 are located so that at time $t$ the vector $\boldsymbol{\rho}=\mathbf{x}_{1}-\mathbf{x}_{2}$ does not extend beyond the cylindrical volume $C_{i, j}$, Figure 2. Let vector $\boldsymbol{\rho}$ has the cylindrical coordinates $b, \varepsilon, \eta$ in the frame of reference with the $z$ axis parallel to the vector $\mathbf{v}=\boldsymbol{\xi}_{1}-\boldsymbol{\xi}_{2}, b$ is the impact parameter, and $\varepsilon$ is the azimuthal angle. If vector $\boldsymbol{\rho}$ belongs to the collision cylinder $C_{i, j}^{+}$, then $0 \leq b \leq d_{i, j}, 0 \leq \varepsilon \leq 2 \pi, d_{i, j} \leq \eta \leq \lambda_{i, j}$. If vector $\boldsymbol{\rho}$ belongs to the collision cylinder $C_{i, j}^{-}$, then $0 \leq b \leq d_{i, j}, 0 \leq \varepsilon \leq 2 \pi,-\lambda_{i, j} \leq \eta \leq-d_{i, j}$. If vector $\boldsymbol{\rho}$ does not extend beyond the interaction domain of two particles $C_{i, j}^{0}$, then $0 \leq b \leq d_{i, j}, 0 \leq \varepsilon \leq 2 \pi,-d_{i, j} \leq \eta \leq d_{i, j}$, Here, $d_{i, j}$ is the range of interaction potential between particles of $i$ - and $j$-quality, and $\lambda_{i, j}$ is the characteristic free path length of particles of $i$ - and $j$-quality.

Generally, any medium particle forms a pair with every other particle. A medium therefore contains $N(N-1) / 2$ pairs of particles. All these pairs are described by the $F_{2(i, j)}\left(t, \mathbf{x}_{1}, \boldsymbol{\xi}_{1}, \mathbf{x}_{2}, \boldsymbol{\xi}_{2}\right)$ function, which obeys the second equation of the BBGKY hierarchy (2.1). If a single particle 2, which either flies away from (Figure 1a) or approaches (Figure 1b) some particle 1, is selected as a partner of this particle, this pair is described by the $F_{2(i, j)}^{p}\left(t, \mathbf{x}_{1}, \xi_{1}, \mathbf{x}_{2}, \xi_{2}\right)$ function. The equation for the $F_{2(i, j)}^{p}\left(t, \mathbf{x}_{1}, \boldsymbol{\xi}_{1}, \mathbf{x}_{2}, \boldsymbol{\xi}_{2}\right)$ function assumes a form, 


$$
\begin{gathered}
\left(\frac{\partial}{\partial t}+\xi_{1} \frac{\partial}{\partial \mathbf{x}_{1}}+\xi_{2} \frac{\partial}{\partial \mathbf{x}_{2}}+\frac{\boldsymbol{\Phi}_{1,2}}{m_{i}} \frac{\partial}{\partial \xi_{1}}+\frac{\boldsymbol{\Phi}_{2,1}}{m_{j}} \frac{\partial}{\partial \xi_{2}}\right) F_{2(i, j)}^{p}\left(t, \mathbf{x}_{1}, \xi_{1}, \mathbf{x}_{2}, \xi_{2}\right)\left(1+\mathrm{O}\left(v_{i, j}\right)\right)=0 \\
F_{2(i, j)}^{p}=F_{2(i, j)}^{p(+)}, \quad \boldsymbol{\rho} \in C_{i, j}^{+} \\
F_{2(i, j)}^{p}=F_{2(i, j)}^{p(-)}, \quad \boldsymbol{\rho} \in C_{i, j}^{-} \\
F_{2(i, j)}^{p}=F_{2(i, j)}^{p(0)}, \quad \boldsymbol{\rho} \in C_{i, j}^{0}
\end{gathered}
$$

Here, $v_{i, j}=d_{i, j} / \lambda_{i, j}$. In Equation (2.2), coordinates and velocities of particles of all the $i, j$-pairs without exception, $i, j=1, \ldots, L$, have identical meanings, $\mathbf{x}_{1}, \mathbf{x}_{2}$ and $\xi_{1}, \xi_{2}$. Equation (2.2) was derived for a pair in a gas of identical structureless particles (Lebed, 2015a). The derivation by Lebed (2015a) remains true for a pair of structureless particles of $i$ - and $j$-quality in a gas mixture $i, j=1, \ldots, L$. Equation (2.2) is valid only in the case of rarefied gas, when $d_{i, j} \ll \lambda_{i, j}$. Equation (2.2) has a clear physical meaning. It asserts that a pair of flying-apart particles, that is, of particles that have already left the domain of their interaction $C_{i, j}^{0}$ and are located within the cylindrical volume $C_{i, j}^{+}$, travels undergoing no collisions with third particle 3 . And a pair of drawing together particles, that is, of particles that have not yet reached the domain of their interaction $C_{i, j}^{0}$ and are located within the cylindrical volume $C_{i, j}^{-}$, travels undergoing no collisions with third particle 3 too. Note that $F_{2(i, j)}^{p(+)}\left(t, \mathbf{x}_{1}, \boldsymbol{\xi}_{1}, \mathbf{x}_{2}, \boldsymbol{\xi}_{2}\right)=0$, when vector $\boldsymbol{\rho}=\mathbf{x}_{1}-\mathbf{x}_{2}$ is not located within the cylindrical volume $C_{i, j}^{+}$, and $F_{2(i, j)}^{p(-)}\left(t, \mathbf{x}_{1}, \boldsymbol{\xi}_{1}, \mathbf{x}_{2}, \boldsymbol{\xi}_{2}\right)=0$, when vector $\boldsymbol{\rho}=\mathbf{x}_{1}-\mathbf{x}_{2}$ is not located within the cylindrical volume $C_{i, j}^{-}$.

Let us switch from coordinates $\mathbf{x}_{1}, \boldsymbol{\xi}_{1}, \mathbf{x}_{2}, \boldsymbol{\xi}_{2}$ of two particles to phase coordinates $\mathbf{x}_{1}, \boldsymbol{\xi}_{1}, \boldsymbol{\rho}=\mathbf{x}_{1}-\mathbf{x}_{2}, \mathbf{v}=\boldsymbol{\xi}_{1}-\boldsymbol{\xi}_{2}$. Then,

$$
F_{2(i, j)}^{p}\left(t, \mathbf{x}_{1}, \boldsymbol{\xi}_{1}, \mathbf{x}_{2}, \boldsymbol{\xi}_{2}\right)=\widehat{\bar{F}}_{2(i, j)}^{p}\left(t, \mathbf{x}_{1}, \boldsymbol{\xi}_{1}, \mathbf{v}, \boldsymbol{\rho}\right)
$$

Let us consider the case when coordinates and velocities of one particle of all the $i, j$-pairs without exception, $i, j=1, \ldots, L$, say $i$-particle, have identical meanings, $\mathbf{x}_{1}, \xi_{1}$. At the same time, coordinates and velocities of $j$-particle of each of the $i, j$-pairs, $\mathbf{x}_{2}^{(i, j)}, \boldsymbol{\xi}_{2}^{(i, j)}$, are chosen so that the mass centre coordinates and the mass centre velocities of all the $i, j$-pairs without exception, $i, j=1, \ldots, L$, have identical meanings, $\mathbf{x}, \mathbf{G}$. Let us switch from phase coordinates $\mathbf{x}_{1}, \boldsymbol{\xi}_{1}, \mathbf{x}_{2}, \boldsymbol{\xi}_{2}$ of two particles to phase coordinates $\mathbf{x}_{1}, \boldsymbol{\xi}_{1}, \mathbf{x}_{2}^{(i, j)}, \boldsymbol{\xi}_{2}^{(i, j)}$,

$$
\begin{gathered}
\xi_{2}^{(i, j)}=\frac{m_{i}+m_{j}}{2 m_{j}} \xi_{2}-\frac{m_{i}-m_{j}}{2 m_{j}} \xi_{1} \xi_{2}^{(i, i)}=\xi_{2} \\
\mathbf{x}_{2}^{(i, j)}=\frac{m_{i}+m_{j}}{2 m_{j}} \mathbf{x}_{2}-\frac{m_{i}-m_{j}}{2 m_{j}} \mathbf{x}_{1} \mathbf{x}_{2}^{(i, i)}=\mathbf{x}_{2}
\end{gathered}
$$

Let us switch from coordinates $\mathbf{x}_{1}, \boldsymbol{\xi}_{1}, \mathbf{x}_{2}^{(i, j)}, \boldsymbol{\xi}_{2}^{(i, j)}$ of two particles first to phase coordinates $\mathbf{x}_{1}, \boldsymbol{\xi}_{1}, \mathbf{v}^{(i, j)}, \boldsymbol{\rho}^{(i, j)}$, and then to phase coordinates $\mathbf{x}, \mathbf{G}, \mathbf{v}^{(i, j)}, \boldsymbol{\rho}^{(i, j)}$,

$$
\begin{aligned}
& \mathbf{x}=\frac{\left(m_{i} \mathbf{x}_{1}+m_{j} \mathbf{x}_{2}^{(i, j)}\right)}{\left(m_{i}+m_{j}\right)} \boldsymbol{\rho}^{(i, j)}=\mathbf{x}_{1}-\mathbf{x}_{2}^{(i, j)} \\
& \mathbf{G}=\frac{\left(m_{i} \xi_{1}+m_{j} \xi_{2}^{(i, j)}\right)}{\left(m_{i}+m_{j}\right)} \mathbf{v}^{(i, j)}=\boldsymbol{\xi}_{1}-\boldsymbol{\xi}_{2}^{(i, j)}
\end{aligned}
$$

Then,

$$
F_{2(i, j)}^{* p}\left(t, \mathbf{x}_{1}, \boldsymbol{\xi}_{1}, \mathbf{x}_{2}^{(i, j)}, \boldsymbol{\xi}_{2}^{(i, j)}\right)=\tilde{\tilde{F}}_{2(i, j)}^{p}\left(t, \mathbf{x}_{1}, \boldsymbol{\xi}_{1}, \mathbf{v}^{(i, j)}, \boldsymbol{\rho}^{(i, j)}\right)=\tilde{F}_{2(i, j)}^{p}\left(t, \mathbf{x}, \mathbf{G}, \mathbf{v}^{(i, j)}, \boldsymbol{\rho}^{(i, j)}\right)
$$

In Equations (2.4), each of the $\mathbf{v}^{(i, j)}\left(\boldsymbol{\rho}^{(i, j)}\right)$ vectors, $i, j=1, \ldots, L$, has the same direction, 


$$
\mathbf{v}^{(i, i)}=\mathbf{v}=\frac{2 m_{j}}{m_{i}+m_{j}} \mathbf{v}^{(i, j)} \boldsymbol{\rho}^{(i, i)}=\boldsymbol{\rho}=\frac{2 m_{j}}{m_{i}+m_{j}} \boldsymbol{\rho}^{(i, j)}
$$

Conservation of the number of imaginary points within the element of phase space yields,

$$
\widehat{\bar{F}}_{2(i, j)}^{p}\left(t, \mathbf{x}_{1}, \boldsymbol{\xi}_{1}, \mathbf{v}, \mathbf{\rho}\right) v b d b d \varepsilon d \mathbf{v}=\tilde{\tilde{F}}_{2(i, j)}^{p}\left(t, \mathbf{x}_{1}, \boldsymbol{\xi}_{1}, \mathbf{v}^{(i, j)}, \mathbf{\rho}^{(i, j)}\right) v^{(i, j)} b^{(i, j)} d b^{(i, j)} d \varepsilon^{(i, j)} d \mathbf{v}^{(i, j)}
$$

Let us consider the case when coordinates $\tilde{\mathbf{x}}_{1}^{(i, j)}, \tilde{\mathbf{x}}_{2}^{(i, j)}$, and velocities $\tilde{\boldsymbol{\xi}}_{1}^{(i, j)}, \tilde{\boldsymbol{\xi}}_{2}^{(i, j)}$, of all the $i-$ and $j$-particles are chosen so that the mass centre coordinates and the mass centre velocities $\mathbf{x}, \mathbf{G}$, and relative motion coordinate and relative motion velocity $\boldsymbol{\rho}, \mathbf{v}$, have identical meanings for all the $i, j$-pairs without exception, $i, j=1, \ldots, L$,

$$
\begin{gathered}
\mathbf{x}=\frac{\left(m_{i} \tilde{\mathbf{x}}_{1}^{(i, j)}+m_{j} \tilde{\mathbf{x}}_{2}^{(i, j)}\right)}{\left(m_{i}+m_{j}\right)} \boldsymbol{\rho}=\tilde{\mathbf{x}}_{1}^{(i, j)}-\tilde{\mathbf{x}}_{2}^{(i, j)} \\
\mathbf{G}=\frac{\left(m_{i} \tilde{\xi}_{1}^{(i, j)}+m_{j} \tilde{\boldsymbol{\xi}}_{2}^{(i, j)}\right)}{\left(m_{i}+m_{j}\right)} \mathbf{v}=\tilde{\boldsymbol{\xi}}_{1}^{(i, j)}-\tilde{\boldsymbol{\xi}}_{2}^{(i, j)}
\end{gathered}
$$

here,

$$
\begin{aligned}
& \tilde{\mathbf{x}}_{1}^{(i, j)}=\mathbf{x}_{1}+\frac{\left(m_{j}-m_{i}\right)}{2\left(m_{i}+m_{j}\right)}\left(\mathbf{x}_{1}-\mathbf{x}_{2}\right) \tilde{\xi}_{1}^{(i, j)}=\boldsymbol{\xi}_{1}+\frac{\left(m_{j}-m_{i}\right)}{2\left(m_{i}+m_{j}\right)}\left(\boldsymbol{\xi}_{1}-\boldsymbol{\xi}_{2}\right) \\
& \tilde{\mathbf{x}}_{2}^{(i, j)}=\mathbf{x}_{2}+\frac{\left(m_{j}-m_{i}\right)}{2\left(m_{i}+m_{j}\right)}\left(\mathbf{x}_{1}-\mathbf{x}_{2}\right) \tilde{\boldsymbol{\xi}}_{2}^{(i, j)}=\boldsymbol{\xi}_{2}+\frac{\left(m_{j}-m_{i}\right)}{2\left(m_{i}+m_{j}\right)}\left(\boldsymbol{\xi}_{1}-\boldsymbol{\xi}_{2}\right)
\end{aligned}
$$

Then,

$$
F_{2(i, j)}^{p}\left(t, \mathbf{x}_{1}, \boldsymbol{\xi}_{1}, \mathbf{x}_{2}, \boldsymbol{\xi}_{2}\right)=F_{2(i, j)}^{* * p}\left(t, \tilde{\mathbf{x}}_{1}^{(i, j)}, \tilde{\boldsymbol{\xi}}_{1}^{(i, j)}, \tilde{\mathbf{x}}_{2}^{(i, j)}, \tilde{\boldsymbol{\xi}}_{2}^{(i, j)}\right)=\widehat{F}_{2(i, j)}^{p}(t, \mathbf{x}, \mathbf{G}, \mathbf{v}, \boldsymbol{\rho})
$$

Conservation of the number of imaginary points within the element of phase space yields,

$$
\hat{F}_{2(i, j)}^{p}(t, \mathbf{x}, \mathbf{G}, \mathbf{v}, \boldsymbol{\rho}) v b d b d \varepsilon d \mathbf{v}=\tilde{F}_{2(i, j)}^{p}\left(t, \mathbf{x}, \mathbf{G}, \mathbf{v}^{(i, j)}, \boldsymbol{\rho}^{(i, j)}\right) v^{(i, j)} b^{(i, j)} d b^{(i, j)} d \varepsilon^{(i, j)} d \mathbf{v}^{(i, j)}
$$

Let us recast the Equation (2.2) first in terms of the $\widehat{\bar{F}}_{2(i, j)}^{p}\left(t, \mathbf{x}_{1}, \boldsymbol{\xi}_{1}, \mathbf{v}, \boldsymbol{\rho}\right)$ function, written in $\mathbf{x}_{1}, \boldsymbol{\xi}_{1}, \boldsymbol{\rho}, \mathbf{v}$ variables,

$$
\left(\frac{\partial}{\partial t}+\xi_{1} \frac{\partial}{\partial \mathbf{x}_{1}}+\mathbf{v} \frac{\partial}{\partial \boldsymbol{\rho}}+\frac{\boldsymbol{\Phi}_{1,2}}{m_{i}} \frac{\partial}{\partial \boldsymbol{\xi}_{1}}+\frac{\boldsymbol{\Phi}_{1,2}}{\mu_{i, j}} \frac{\partial}{\partial \mathbf{v}}\right) \overline{\hat{F}}_{2(i, j)}^{p}\left(t, \mathbf{x}_{1}, \boldsymbol{\xi}_{1}, \mathbf{v}, \boldsymbol{\rho}\right)\left(1+\mathrm{O}\left(v_{i, j}\right)\right)=0
$$

and then in terms of the $\hat{F}_{2(i, j)}^{p}(t, \mathbf{x}, \mathbf{G}, \mathbf{v}, \boldsymbol{\rho})$ function, written in $\mathbf{x}, \mathbf{G}, \mathbf{v}, \boldsymbol{\rho}$ variables,

$$
\left(\frac{\partial}{\partial t}+\mathbf{G} \frac{\partial}{\partial \mathbf{x}}+\mathbf{v} \frac{\partial}{\partial \boldsymbol{\rho}}+\frac{\boldsymbol{\Phi}_{1,2}}{\mu_{i, j}} \frac{\partial}{\partial \mathbf{v}}\right) \hat{F}_{2(i, j)}^{p}(t, \mathbf{x}, \mathbf{G}, \mathbf{v}, \boldsymbol{\rho})\left(1+\mathrm{O}\left(v_{i, j}\right)\right)=0
$$

In Equations (2.9) and (2.10), $\mu_{i, j}=m_{i} m_{j} /\left(m_{i}+m_{j}\right)$. Let us recast Equation (2.2) first in terms of the $\tilde{F}_{2(i, j)}^{p}\left(t, \mathbf{x}_{1}, \boldsymbol{\xi}_{1}, \mathbf{v}^{(i, j)}, \boldsymbol{\rho}^{(i, j)}\right)$ function, written in $\mathbf{x}_{1}, \boldsymbol{\xi}_{1}, \mathbf{v}^{(i, j)}, \boldsymbol{\rho}^{(i, j)}$ variables,

$$
\left(\frac{\partial}{\partial t}+\xi_{1} \frac{\partial}{\partial \mathbf{x}_{1}}+\mathbf{v}^{(i, j)} \frac{\partial}{\partial \boldsymbol{\rho}^{(i, j)}}+\frac{\boldsymbol{\Phi}_{1,2}}{m_{i}} \frac{\partial}{\partial \xi_{1}}+\frac{\boldsymbol{\Phi}_{1,2}}{\mu_{i, j}} \frac{\partial}{\partial \mathbf{v}^{(i, j)}}\right) \tilde{\tilde{F}}_{2(i, j)}^{p}\left(t, \mathbf{x}_{1}, \boldsymbol{\xi}_{1}, \mathbf{v}^{(i, j)}, \boldsymbol{\rho}^{(i, j)}\right)\left(1+\mathrm{O}\left(v_{i, j}\right)\right)=0
$$

and then in terms of the $\tilde{F}_{2(i, j)}^{p}\left(t, \mathbf{x}, \mathbf{G}, \mathbf{v}^{(i, j)}, \boldsymbol{\rho}^{(i, j)}\right)$ function, written in $\mathbf{x}, \mathbf{G}, \mathbf{v}^{(i, j)}, \boldsymbol{\rho}^{(i, j)}$ variables, 


$$
\left(\frac{\partial}{\partial t}+\mathbf{G} \frac{\partial}{\partial \mathbf{x}}+\mathbf{v}^{(i, j)} \frac{\partial}{\partial \boldsymbol{\rho}^{(i, j)}}+\frac{\mathbf{\Phi}_{1,2}}{\mu_{i, j}} \frac{\partial}{\partial \mathbf{v}^{(i, j)}}\right) \tilde{F}_{2(i, j)}^{p}\left(t, \mathbf{x}, \mathbf{G}, \mathbf{v}^{(i, j)}, \boldsymbol{\rho}^{(i, j)}\right)\left(1+\mathrm{O}\left(v_{i, j}\right)\right)=0
$$

Consider phase coordinates $\breve{\mathbf{x}}_{1}^{(i, j)}, \breve{\xi}_{1}^{(i, j)}, \breve{\mathbf{x}}_{2}^{(i, j)}, \breve{\xi}_{2}^{(i, j)}$, so that, $\mathbf{x}_{1}, \boldsymbol{\xi}_{1}, \mathbf{x}_{2}^{(i, j)}, \boldsymbol{\xi}_{2}^{(i, j)}$. Let us specify more common phase coordinates

$$
\mathbf{G}=\frac{\left(m_{i} \breve{\xi}_{1}^{(i, j)}+m_{j} \breve{\xi}_{2}^{(i, j)}\right)}{\left(m_{i}+m_{j}\right)} \mathbf{x}=\frac{\left(m_{i} \breve{\mathbf{x}}_{1}^{(i, j)}+m_{j} \breve{\mathbf{x}}_{2}^{(i, j)}\right)}{\left(m_{i}+m_{j}\right)}
$$

Let us recast Equation (2.2) in terms of the $\breve{F}_{2(i, j)}^{p}\left(t, \breve{\mathbf{x}}_{1}^{(i, j)}, \breve{\boldsymbol{\xi}}_{1}^{(i, j)}, \breve{\mathbf{v}}^{(i, j)}, \breve{\boldsymbol{\rho}}^{(i, j)}\right)$ function, written in $\breve{\mathbf{x}}_{1}^{(i, j)}, \breve{\boldsymbol{\xi}}_{1}^{(i, j)}, \breve{\mathbf{v}}^{(i, j)}=\breve{\boldsymbol{\xi}}_{1}^{(i, j)}-\breve{\boldsymbol{\xi}}_{2}^{(i, j)}, \breve{\boldsymbol{\rho}}^{(i, j)}=\breve{\mathbf{x}}_{1}^{(i, j)}-\breve{\mathbf{x}}_{2}^{(i, j)} \quad$ variables,

$$
\begin{gathered}
\left(\frac{\partial}{\partial t}+\breve{\xi}_{1}^{(i, j)} \frac{\partial}{\partial \breve{\mathbf{x}}_{1}^{(i, j)}}+\breve{\mathbf{v}}^{(i, j)} \frac{\partial}{\partial \breve{\boldsymbol{\rho}}^{(i, j)}}+\frac{\boldsymbol{\Phi}_{1,2}}{m_{i}} \frac{\partial}{\partial \breve{\xi}_{1}^{(i, j)}}+\frac{\boldsymbol{\Phi}_{1,2}}{\mu_{i, j}} \frac{\partial}{\partial \breve{\mathbf{v}}^{(i, j)}}\right) \times \\
\times \breve{F}_{2(i, j)}^{p}\left(t, \breve{\mathbf{x}}_{1}^{(i, j)}, \breve{\boldsymbol{\xi}}_{1}^{(i, j)}, \breve{\mathbf{v}}^{(i, j)}, \breve{\boldsymbol{\rho}}^{(i, j)}\right)\left(1+\mathrm{O}\left(v_{i, j}\right)\right)=0
\end{gathered}
$$

In particular case,

$$
\breve{F}_{2(i, j)}^{p}\left(t, \breve{\mathbf{x}}_{1}^{(i, j)}=\mathbf{x}_{1}, \breve{\xi}_{1}^{(i, j)}=\boldsymbol{\xi}_{1}, \breve{\mathbf{v}}^{(i, j)}=\mathbf{v}^{(i, j)}, \breve{\boldsymbol{\rho}}^{(i, j)}=\boldsymbol{\rho}^{(i, j)}\right)=\tilde{\tilde{F}}_{2(i, j)}^{p}\left(t, \mathbf{x}_{1}, \boldsymbol{\xi}_{1}, \mathbf{v}^{(i, j)}, \boldsymbol{\rho}^{(i, j)}\right)
$$

The solutions to Equations (2.2), (2.9), (2.10), (2.11), (2.12), and (2.13) describe the system both at the initial $l_{0}$-stage and the kinetic $l_{k}$-stage. However, these solutions are not capable of describing the system at the hydrodynamic $l_{h}$-stage. The reason is as follows. Let at time $t$ particles of $i$ - and $j$-quality freely flying apart after interaction with each other are separated by the limiting intermolecular distance $\eta=\lambda_{i, j}$. This, generally, does not imply that at time $t$ the particle of $i$-quality enters into interaction with some other particle of $j$-quality. However, at time $t$ the particle of $i$-quality inevitably enter into interaction with some other particle of $k$-quality, $k=1, \ldots, L$. Analogously, let at time $t$ particles of $i$ - and $j$-quality approaching toward the interaction with each other are separated by the limiting intermolecular distance $\eta=-\lambda_{i, j}$. This, generally, does not imply that at time $t$ the particle of $i-$ quality leaves the interaction with some other particle of $j$-quality. However, at time $t$ the particle of $i$-quality inevitably leaves the interaction with some other particle of $k$-quality, $k=1, \ldots, L$.

Let us consider the collision cylinders $C_{i, j}^{+}$and $C_{i, j}^{-}$. Let us sum up Equation (2.2) with respect to $j$, $j=1, \ldots, L$, using weight function $N_{i} \tilde{N}_{j}, N_{i} \tilde{N}_{j}=N_{i}\left(N_{j}-1\right)$ for $j=i, N_{i} \tilde{N}_{j}=N_{i} N_{j} / 2$ for $j \neq i$,

$$
\sum_{j=1}^{L}\left(\frac{\partial}{\partial t}+\xi_{1} \frac{\partial}{\partial \mathbf{x}_{1}}+\xi_{2} \frac{\partial}{\partial \mathbf{x}_{2}}\right) N_{i} \tilde{N}_{j} F_{2(i, j)}^{p( \pm)}\left(t, \mathbf{x}_{1}, \boldsymbol{\xi}_{1}, \mathbf{x}_{2}, \boldsymbol{\xi}_{2}\right)\left(1+\mathrm{O}\left(v_{i, j}\right)\right)=0
$$

Within the collision cylinders $C_{i, j}^{+}$and $C_{i, j}^{-}$, the solutions to Equations (2.15) are capable of describing the system at the hydrodynamic $l_{h}$-stage. In analogous, let us sum up Equations (2.9) and (2.10) with respect to $j, j=1, \ldots, L$, using weight function $N_{i} \tilde{N}_{j}$,

$$
\begin{aligned}
& \sum_{j=1}^{L}\left(\frac{\partial}{\partial t}+\xi_{1} \frac{\partial}{\partial \mathbf{x}_{1}}+\mathbf{v} \frac{\partial}{\partial \boldsymbol{\rho}}\right) N_{i} \tilde{N}_{j} \hat{\bar{F}}_{2(i, j)}^{p( \pm)}\left(t, \mathbf{x}_{1}, \boldsymbol{\xi}_{1}, \mathbf{v}, \boldsymbol{\rho}\right)\left(1+\mathrm{O}\left(v_{i, j}\right)\right)=0 \\
& \sum_{j=1}^{L}\left(\frac{\partial}{\partial t}+\mathbf{G} \frac{\partial}{\partial \mathbf{x}}+\mathbf{v} \frac{\partial}{\partial \boldsymbol{\rho}}\right) N_{i} \tilde{N}_{j} \widehat{F}_{2(i, j)}^{p( \pm)}(t, \mathbf{x}, \mathbf{G}, \mathbf{v}, \boldsymbol{\rho})\left(1+\mathrm{O}\left(v_{i, j}\right)\right)=0
\end{aligned}
$$

Within the collision cylinders $C_{i, j}^{+}$and $C_{i, j}^{-}$, the solutions to Equations (2.16) and (2.17) are capable of describing the system at the hydrodynamic $l_{h}$-stage. The solutions to Equation (2.15) are valid for the case when coordinates and velocities of particles of all the $i, j$-pairs without exception, $i, j=1, \ldots, L$, have identical arbitrary meanings, $\mathbf{x}_{1}, \xi_{1}$ and $\mathbf{x}_{2}, \xi_{2}$. The solutions to Equation (2.16) are valid for the case when $i$ - particle coordinate and velocity, and relative motion coordinate and velocity of all the $i, j$-pairs without exception, $i, j=1, \ldots, L$, have identical arbitrary meanings, $\mathbf{x}_{1}, \xi_{1}$ and $\boldsymbol{\rho}, \mathbf{v}$. The solutions to Equation (2.17) are valid for the case when mass centre coordinate and velocity, and relative motion coordinate and velocity of all the $i, j$-pairs without exception, $i, j=1, \ldots, L$, have identical arbitrary meanings, $\mathbf{x}, \mathbf{G}$ and $\boldsymbol{\rho}, \mathbf{v}$. 
Conditions $\boldsymbol{\rho} \in C_{i, j}^{+}$and $\boldsymbol{\rho} \in C_{i, j}^{-}$impose restrictions on the arbitrary choice of meanings of $\mathbf{x}_{1}$ and $\mathbf{x}_{2}$, $\tilde{\mathbf{x}}_{1}^{(i, j)}$ and $\tilde{\mathbf{x}}_{2}^{(i, j)}$.

\section{Relationships between Distribution Functions}

Let us set up frame of reference, such that the z-axis is parallel to $\mathbf{v}^{(i, j)}$ (see, Figure 2 with $\mathbf{v}=\mathbf{v}^{(i, j)}$ and $\left.\boldsymbol{\rho}=\boldsymbol{\rho}^{(i, j)}\right)$. Let $b, \varepsilon$, and $\eta$ be the cylindrical coordinates of vector $\boldsymbol{\rho}^{(i, j)}$ in this frame of reference. Functions $F_{p(i, j)}^{d i v}, F_{p(i, j)}^{a p p}$ and $F_{p p(i, j)}^{d i v}, F_{p p(i, j)}^{a p p}$ are defined as,

$$
\begin{gathered}
F_{p(i, j)}^{d i v}\left(t, \mathbf{x}, \mathbf{G}, \mathbf{v}^{(i, j)}\right)=\int_{0}^{2 \pi} \int_{0}^{d_{i, j}} F_{p(i, j)}^{d i v}\left(t, \mathbf{x}, \mathbf{G}, \mathbf{v}^{(i, j)}, b, \varepsilon\right) b d b d \varepsilon= \\
=\int_{d_{i, j}}^{\lambda_{i, j}} \int_{0}^{2 \pi} \int_{0}^{d_{i, j}} \tilde{F}_{2(i, j)}^{p(+)}\left(t, \mathbf{x}, \mathbf{G}, \mathbf{v}^{(i, j)}, b, \varepsilon, \eta\right) b d b d \varepsilon d \eta \\
F_{p(i, j)}^{a p p}\left(t, \mathbf{x}, \mathbf{G}, \mathbf{v}^{(i, j)}\right)=\int_{0}^{2 \pi} \int_{0}^{d_{i, j}} F_{p(i, j)}^{a p p}\left(t, \mathbf{x}, \mathbf{G}, \mathbf{v}^{(i, j)}, b, \varepsilon\right) b d b d \varepsilon= \\
=\int_{-\lambda_{i, j}}^{-d_{i, j}} \int_{0}^{2 \pi} \int_{0}^{d_{i, j}} \tilde{F}_{2(i, j)}^{p(-)}\left(t, \mathbf{x}, \mathbf{G}, \mathbf{v}^{(i, j)}, b, \varepsilon, \eta\right) b d b d \varepsilon d \eta \\
F_{p p(i, j)}^{d i v}\left(t, \mathbf{x}_{1}, \boldsymbol{\xi}_{1}, \mathbf{v}^{(i, j)}\right)=\int_{0}^{2 \pi} \int_{0}^{d_{i, j}} F_{p p(i, j)}^{d i v}\left(t, \mathbf{x}_{1}, \boldsymbol{\xi}_{1}, \mathbf{v}^{(i, j)}, b, \varepsilon\right) b d b d \varepsilon= \\
=\int_{d_{i, j}}^{\lambda_{i, j}} \int_{0}^{2 \pi} \int_{0}^{d_{i, j}} \tilde{F}_{2(i, j)}^{p(+)}\left(t, \mathbf{x}_{1}, \boldsymbol{\xi}_{1}, \mathbf{v}^{(i, j)}, b, \varepsilon, \eta\right) b d b d \varepsilon d \eta \\
F_{p p(i, j)}^{a p p}\left(t, \mathbf{x}_{1}, \boldsymbol{\xi}_{1}, \mathbf{v}^{(i, j)}\right)=\int_{0}^{2 \pi} \int_{0}^{d_{i, j}} F_{p p(i, j)}^{a p p}\left(t, \mathbf{x}_{1}, \boldsymbol{\xi}_{1}, \mathbf{v}^{(i, j)}, b, \varepsilon\right) b d b d \varepsilon= \\
d_{i, j} \tilde{F}_{2(i, j)}^{p(-)}\left(t, \mathbf{x}_{1}, \boldsymbol{\xi}_{1}, \mathbf{v}^{(i, j)}, b, \varepsilon, \eta\right) b d b d \varepsilon d \eta
\end{gathered}
$$

In accordance with Lebed (1996a), the $F_{p(i, j)}^{d i v}, F_{p(i, j)}^{a p p}$ functions will be called pair distribution functions. Pairs of particles modeled by the $F_{p(i, j)}^{d i v}, F_{p(i, j)}^{a p p}$ functions have their centers of mass in fixed positions $(\mathbf{x})$, and particles composing a pair are so arranged in the cylindrical volumes $C_{i, j}^{+}$and $C_{i, j}^{-}$that their coordinates comply with the integration limits in Equations (3.1). In pairs described by the $F_{p p(i, j)}^{d i v}, F_{p p(i, j)}^{a p p}$ functions, $i$-particle is fixed $\left(\mathbf{x}_{1}\right)$, and position of $j$-particle in the cylindrical volumes $C_{i, j}^{+}$and $C_{i, j}^{-}$is limited by the integration limits in Equations (3.1). In accordance with Lebed (1996a), the $F_{p p(i, j)}^{F^{d i v}}, F_{p p(i, j)}^{a p p}$ functions will be called paired-particle distribution functions. In the space regions bounded by integration limits (3.1), force terms (2.11), (2.12) should be omitted. The result is that functions $\tilde{F}_{2(i, j)}^{p}, \tilde{\tilde{F}}_{2(i, j)}^{p}$ at any point $d_{i, j} \leq \eta \leq \lambda_{i, j}$ and $-\lambda_{i, j} \leq \eta \leq-d_{i, j}$ can be expressed in terms of functions at extreme points $\eta \pm d_{i, j}$ and $\eta \pm \lambda_{i, j}$. In particular,

$$
\begin{aligned}
& \tilde{F}_{2(i, j)}^{p(+)}\left(t, \mathbf{x}, \mathbf{G}, \mathbf{v}^{(i, j)}, b, \varepsilon, \eta\right)=\tilde{F}_{2(i, j)}^{p(+)}\left(t-\frac{\left(\eta-d_{i, j}\right)}{v^{(i, j)}}, \mathbf{x}-\mathbf{G} \frac{\left(\eta-d_{i, j}\right)}{v^{(i, j)}}, \mathbf{G}, \mathbf{v}^{(i, j)}, b, \varepsilon, \eta=+d_{i, j}\right) \\
& \tilde{\tilde{F}}_{2(i, j)}^{p(+)}\left(t, \mathbf{x}_{1}, \boldsymbol{\xi}_{1}, \mathbf{v}^{(i, j)}, b, \varepsilon, \eta\right)=\tilde{\tilde{F}}_{2(i, j)}^{p(+)}\left(t-\frac{\left(\eta-d_{i, j}\right)}{v^{(i, j)}}, \mathbf{x}_{1}-\boldsymbol{\xi}_{1} \frac{\left(\eta-d_{i, j}\right)}{v^{(i, j)}}, \boldsymbol{\xi}_{1}, \mathbf{v}^{(i, j)}, b, \varepsilon, \eta=+d_{i, j}\right)
\end{aligned}
$$

In Equations (3.2), $d_{i, j} \leq \eta \leq \lambda_{i, j}$. Now we expand the right hand sides of Equations (3.2) as a series in terms of $\eta$ and substitute the resulting expansions in Equations (3.1). Integration with respect to $\eta$ yields, 


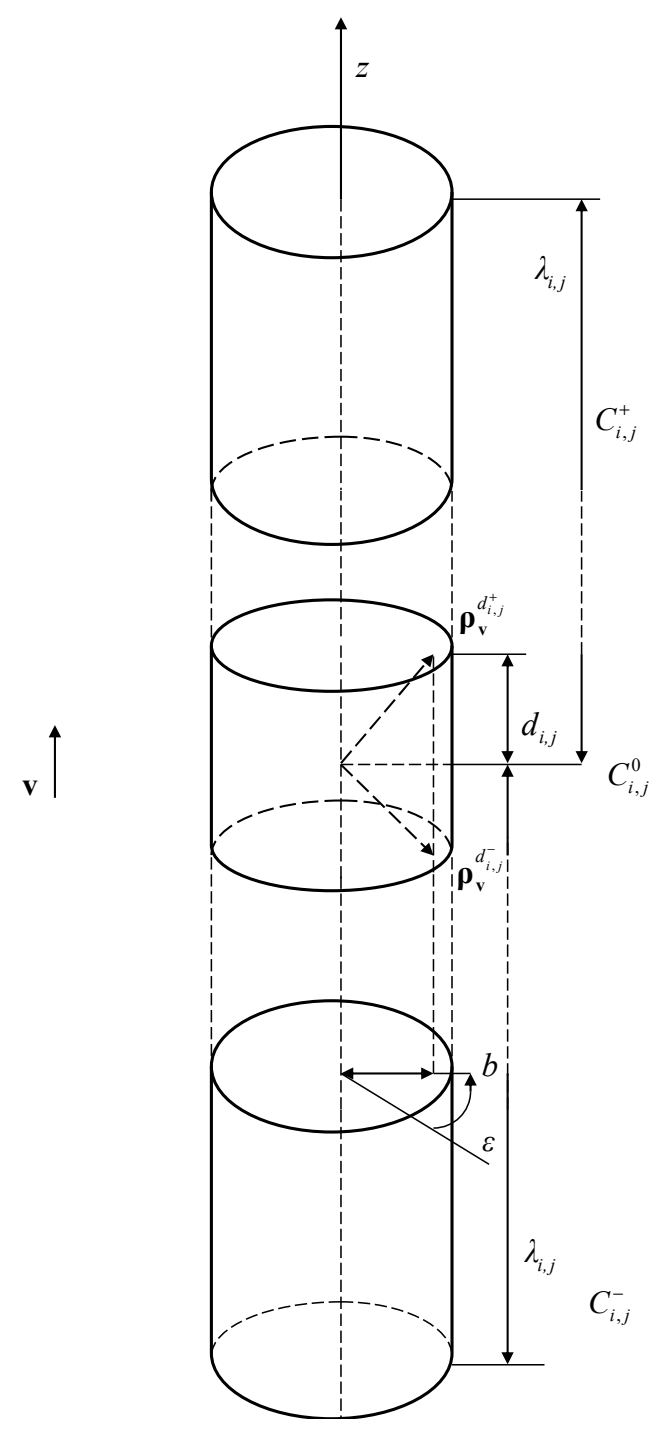

Figure 2. The interaction domain of a pair of particles $C_{i, j}^{0}$, the collision cylinders $C_{i, j}^{-}$and $C_{i, j}^{+}$.

$$
\begin{gathered}
F_{p(i, j)}^{d i v}\left(t, \mathbf{x}, \mathbf{G}, \mathbf{v}^{(i, j)}, b, \varepsilon\right)=\tau_{i, j} v^{(i, j)}\left(1-\frac{\tau_{i, j}}{2} \nabla+\ldots\right) \tilde{F}_{2(i, j)}^{p(+)}\left(t, \mathbf{x}, \mathbf{G}, \mathbf{v}^{(i, j)}, b, \varepsilon, \eta=+d_{i, j}\right) \\
F_{p p(i, j)}^{d i v}\left(t, \mathbf{x}_{1}, \xi_{1}, \mathbf{v}^{(i, j)}, b, \varepsilon\right)=\tau_{i, j} v^{(i, j)}\left(1-\frac{\tau_{i, j}}{2} \nabla_{1}+\ldots\right) \tilde{F}_{2(i, j)}^{p(+)}\left(t, \mathbf{x}_{1}, \xi_{1}, \mathbf{v}^{(i, j)}, b, \varepsilon, \eta=+d_{i, j}\right)
\end{gathered}
$$

In Equations (3.3),

$$
\tau_{i, j}=\left(\lambda_{i, j}-d_{i, j}\right) / v \nabla=\frac{\partial}{\partial t}+\mathbf{G} \frac{\partial}{\partial \mathbf{x}} \nabla_{1}=\frac{\partial}{\partial t}+\xi_{1} \frac{\partial}{\partial \mathbf{x}_{1}}
$$

By analogy with Equations (3.2) and (3.3),

$$
\begin{gathered}
F_{p(i, j)}^{a p p}\left(t, \mathbf{x}, \mathbf{G}, \mathbf{v}^{(i, j)}, b, \varepsilon\right)=\tau_{i, j} v^{(i, j)}\left(1+\frac{\tau_{i, j}}{2} \nabla+\ldots\right) \tilde{F}_{2(i, j)}^{p(-)}\left(t, \mathbf{x}, \mathbf{G}, \mathbf{v}^{(i, j)}, b, \varepsilon, \eta=-d_{i, j}\right) \\
F_{p p(i, j)}^{a p p}\left(t, \mathbf{x}_{1}, \xi_{1}, \mathbf{v}^{(i, j)}, b, \varepsilon\right)=\tau_{i, j} v^{(i, j)}\left(1+\frac{\tau_{i, j}}{2} \nabla_{1}+\ldots\right) \tilde{F}_{2(i, j)}^{p(-)}\left(t, \mathbf{x}_{1}, \xi_{1}, \mathbf{v}^{(i, j)}, b, \varepsilon, \eta=-d_{i, j}\right)
\end{gathered}
$$

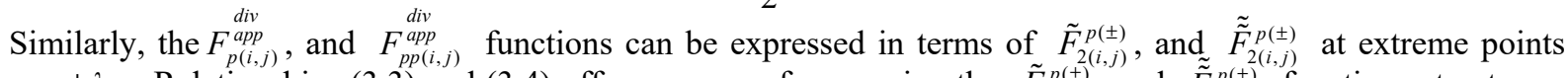
$\eta= \pm \lambda_{i, j}$. Relationships (3.3) and (3.4) offer a means of expressing the $\tilde{F}_{2(i, j)}^{p(t)}$, and $\tilde{\tilde{F}}_{2(i, j)}^{p( \pm)}$ functions at extreme points $\eta= \pm d$ in terms of pair and paired-particle distribution functions. From Equations (3.3) and (3.4) one obtains through repeated substitutions, 


$$
\begin{gathered}
\tilde{F}_{2(i, j)}^{p( \pm)}\left(t, \mathbf{x}, \mathbf{G}, \mathbf{v}^{(i, j)}, b, \varepsilon, \eta= \pm d_{i, j}\right)=\frac{1}{\tau_{i, j} v^{(i, j)}}\left(1 \pm \frac{\tau_{i, j}}{2} \nabla+\ldots\right) F_{p(i, j)}^{d i v}\left(t, \mathbf{x}, \mathbf{G}, \mathbf{v}^{(i, j)}, b, \varepsilon\right) \\
\tilde{\tilde{F}}_{2(i, j)}^{p( \pm)}\left(t, \mathbf{x}_{1}, \boldsymbol{\xi}_{1}, \mathbf{v}^{(i, j)}, b, \varepsilon, \eta= \pm d_{i, j}\right)=\frac{1}{\tau_{i, j} v^{(i, j)}}\left(1 \pm \frac{\tau_{i, j}}{2} \nabla_{1}+\ldots\right) F_{p p(i, j)}^{a i v}\left(t, \mathbf{x}_{1}, \xi_{1}, \mathbf{v}^{(i, j)}, b, \varepsilon\right)
\end{gathered}
$$

The Equations for the $\tilde{F}_{2(i, j)}^{p( \pm)}$ and $\tilde{\tilde{F}}_{2(i, j)}^{p( \pm)}$ functions at extreme points $\eta \pm \lambda_{i, j}$ may be derived similarly,

$$
\begin{gathered}
\tilde{F}_{2(i, j)}^{p( \pm)}\left(t, \mathbf{x}, \mathbf{G}, \mathbf{v}^{(i, j)}, b, \varepsilon, \eta= \pm \lambda_{i, j}\right)=\frac{1}{\tau_{i, j} v^{(i, j)}}\left(1 \mp \frac{\tau_{i, j}}{2} \nabla+\ldots\right) F_{p(i, j)}^{\text {div }^{a p p}}\left(t, \mathbf{x}, \mathbf{G}, \mathbf{v}^{(i, j)}, b, \varepsilon\right) \\
\tilde{\tilde{F}}_{2(i, j)}^{p( \pm)}\left(t, \mathbf{x}_{1}, \xi_{1}, \mathbf{v}^{(i, j)}, b, \varepsilon, \eta= \pm \lambda_{i, j}\right)=\frac{1}{\tau_{i, j} v^{(i, j)}}\left(1 \mp \frac{\tau_{i, j}}{2} \nabla_{1}+\ldots\right) F_{p p(i, j)}^{d i v}\left(t, \mathbf{x}_{1}, \xi_{1}, \mathbf{v}^{(i, j)}, b, \varepsilon\right)
\end{gathered}
$$

It is convincingly demonstrated that pair distribution functions $F_{p}^{d i v}$ and paired-particle distribution functions $F_{p p}^{d i v} \quad$ slightly vary with time on the initial scale along its trajectories in a gas of identical structureless particles (Lebed, 1996a). These conclusions remain true for a pair of structureless particles of $i$ - and $j$-quality in a gas mixture, $i, j=1, \ldots, L$. In accordance with Lebed (1996a), functions $F_{p(i, j)}^{a p p}\left(t, \mathbf{x}, \mathbf{G}, \mathbf{v}^{(i, j)}, b, \varepsilon\right)$, and $\tilde{F}_{2(i, j)}^{p(+)}\left(t, \mathbf{x}, \mathbf{G}, \mathbf{v}^{(i, j)}, b, \varepsilon, \eta\right)$ for $d_{i, j} \leq \eta \leq \lambda_{i, j}$, and $\tilde{F}_{2(i, j)}^{p(-)}\left(t, \mathbf{x}, \mathbf{G}, \mathbf{v}^{(i, j)}, b, \varepsilon, \eta\right)$ for $-\lambda_{i, j} \leq \eta \leq-d_{i, j}$, slightly vary with time on the initial scale along the trajectory of a center of mass, while functions ${ }_{\tilde{F}_{p p(i, j)}}^{\operatorname{div}}\left(t, \mathbf{x}_{1}, \boldsymbol{\xi}_{1}, \mathbf{v}^{(i, j)}, b, \varepsilon\right)$, and $\tilde{\tilde{F}}_{2(i, j)}^{p(+)}\left(t, \mathbf{x}_{1}, \boldsymbol{\xi}_{1}, \mathbf{v}^{(i, j)}, b, \varepsilon, \eta\right)$ for $d_{i, j} \leq \eta \leq \lambda_{i, j}$, and $\tilde{\tilde{F}}_{2(i, j)}^{p(-)}\left(t, \mathbf{x}_{1}, \boldsymbol{\xi}_{1}, \mathbf{v}^{(i, j)}, b, \varepsilon, \eta\right)$ for $-\lambda_{i, j} \leq \eta \leq-d_{i, j}$, slightly vary with time on the same scale along the particle trajectory. This, generally, does not imply that on the initial scale, these functions slightly vary with space and time separately.

Let us introduce an operation of space averaging of functions within region $S$ with characteristic linear size $l$, such that $d_{i, j} \ll l \ll \lambda_{i, j}$,

$$
\begin{gathered}
\bar{F}_{p(i, j)}^{d i v}\left(t, \mathbf{x}, \mathbf{G}, \mathbf{v}^{(i, j)}, b, \varepsilon\right)=\frac{1}{l^{3}} \int_{S} F_{p(i, j)}^{d i v}\left(t, \mathbf{x}+\mathbf{a}, \mathbf{G}, \mathbf{v}^{(i, j)}, b, \varepsilon\right) d \mathbf{a}= \\
=\frac{1}{l^{3}} \int_{S} \int_{d_{i, j}}^{\lambda_{i, j}} \tilde{F}_{2(i, j)}^{p(+)}\left(t, \mathbf{x}+\mathbf{a}, \mathbf{G}, \mathbf{v}^{(i, j)}, b, \varepsilon, \eta\right) d \eta d \mathbf{a} \\
\bar{F}_{p(i, j)}^{a p p}\left(t, \mathbf{x}, \mathbf{G}, \mathbf{v}^{(i, j)}, b, \varepsilon\right)=\frac{1}{l^{3}} \int_{S} F_{p(i, j)}^{a p p}\left(t, \mathbf{x}+\mathbf{a}, \mathbf{G}, \mathbf{v}^{(i, j)}, b, \varepsilon\right) d \mathbf{a}= \\
=\frac{1}{l^{3}} \int_{S} \int_{-\lambda_{i, j}}^{-d_{i, j}} \tilde{F}_{2(i, j)}^{p(-)}\left(t, \mathbf{x}+\mathbf{a}, \mathbf{G}, \mathbf{v}^{(i, j)}, b, \varepsilon, \eta\right) d \eta d \mathbf{a} \\
\bar{F}_{p p(i, j)}^{d i v}\left(t, \mathbf{x}_{1}, \xi_{1}, \mathbf{v}^{(i, j)}, b, \varepsilon\right)=\frac{1}{l^{3}} \int_{S} F_{p p(i, j)}^{d i v}\left(t, \mathbf{x}_{1}+\mathbf{a}, \xi_{1}, \mathbf{v}^{(i, j)}, b, \varepsilon\right) d \mathbf{a}= \\
=\frac{1}{l^{3}} \int_{S} \int_{d_{i, j}}^{\lambda_{i, j}} \tilde{F}_{2(i, j)}^{p(+)}\left(t, \mathbf{x}_{1}+\mathbf{a}, \xi_{1}, \mathbf{v}^{(i, j)}, b, \varepsilon, \eta\right) d \eta d \mathbf{a} \\
\bar{F}_{p p(i, j)}^{a p p}\left(t, \mathbf{x}_{1}, \xi_{1}, \mathbf{v}^{(i, j)}, b, \varepsilon\right)=\frac{1}{l^{3}} \int_{S} F_{p p(i, j)}^{a p p}\left(t, \mathbf{x}_{1}+\mathbf{a}, \xi_{1}, \mathbf{v}^{(i, j)}, b, \varepsilon\right) d \mathbf{a}= \\
l_{S}^{3} \int_{-\lambda_{i, j}}^{-d_{i, j}} \tilde{F}_{2(i, j)}^{p(-)}\left(t, \mathbf{x}_{1}+\mathbf{a}, \xi_{1}, \mathbf{v}^{(i, j)}, b, \varepsilon, \eta\right) d \eta d \mathbf{a}
\end{gathered}
$$


In view of the aforesaid, space averaged functions (3.7) slightly vary on the initial scale with space and time separately.

To establish correlations between pair and paired-particle distribution functions, the $\tilde{F}_{2(i, j)}^{p(+)}$ function should be expanded as a series of $\mathbf{x}$ in the vicinity of point $\mathbf{x}_{1}$. Let Equation (2.4) be taken into account, then,

$$
\begin{gathered}
\tilde{\tilde{F}}_{2(i, j)}^{p(+)}\left(t, \mathbf{x}_{1}, \boldsymbol{\xi}_{1}, \mathbf{v}^{(i, j)}, \boldsymbol{\rho}^{(i, j)}\right)=\tilde{F}_{2(i, j)}^{p(+)}\left(t, \mathbf{x}=\mathbf{x}_{1}-\frac{m_{j}}{\left(m_{i}+m_{j}\right)} \boldsymbol{\rho}^{(i, j)}, \mathbf{G}=\boldsymbol{\xi}_{1}-\frac{m_{j}}{\left(m_{i}+m_{j}\right)} \mathbf{v}^{(i, j)}, \mathbf{v}^{(i, j)}, \boldsymbol{\rho}^{(i, j)}\right)= \\
=\left.\left(1-\frac{m_{j}}{\left(m_{i}+m_{j}\right)} \boldsymbol{\rho}^{(i, j)} \frac{\partial}{\partial \mathbf{x}}+\ldots\right) \tilde{F}_{2(i, j)}^{p(t)}\left(t, \mathbf{x}, \mathbf{G}, \mathbf{v}^{(i, j)}, \boldsymbol{\rho}^{(i, j)}\right)\right|_{\mathbf{x}=\mathbf{x}_{1}}
\end{gathered}
$$

Let $\eta, \kappa, \omega$ be the coordinates of vector $\boldsymbol{\rho}^{(i, j)}$ in the Cartesian frame of reference, such that the z-axis is parallel to vector $\mathbf{v}$. Now, let us integrate Equation (3.8) with respect to $\boldsymbol{\rho}^{(i, j)}$ within cylindrical volume $C_{i, j}^{+}$, using Equation (3.2), and average the result over space region $S$,

$$
\begin{gathered}
\bar{F}_{p p(i, j)}^{d i v}\left(t, \mathbf{x}_{1}, \xi_{1}, \mathbf{v}^{(i, j)}\right)=\frac{1}{l^{3}} \int_{S} \int_{S_{i, j}^{+}}\left(1-\frac{m_{j}}{\left(m_{i}+m_{j}\right)} \eta \frac{\partial}{\partial x_{\eta}}-\frac{m_{j}}{\left(m_{i}+m_{j}\right)} \kappa \frac{\partial}{\partial x_{\kappa}}-\frac{m_{j}}{\left(m_{i}+m_{j}\right)} \omega \frac{\partial}{\partial x_{\omega}}+\ldots\right) \times \\
\times\left.\left(1-\frac{\left(\eta-d_{i, j}\right)}{v^{(i, j)}} \nabla+\ldots\right) \tilde{F}_{2(i, j)}^{p(+)}\left(t, \mathbf{x}+\mathbf{a}, \mathbf{G}, \mathbf{v}^{(i, j)}, b, \varepsilon, \eta=+d\right)\right|_{\mathbf{x}=\mathbf{x}_{1}} \quad b d b d \varepsilon d \eta d \mathbf{a}
\end{gathered}
$$

Values $x_{\eta}, x_{\kappa}, x_{\omega}$ appearing in Equation (3.9) are the projections of vector $\mathbf{x}$ onto the axes of the Cartesian frame of reference. The $\tilde{F}_{2(i, j)}^{p(+)}$ function vary insignificantly on the initial scale, while the limits of integration with respect to $\kappa$ and $\omega$ do not exceed $d_{i, j}$. This implies that the terms on the right hand side of Equation (3.9) that are proportional to $\kappa$ and $\omega$ may be dropped out, because their ratio to the remaining terms is a small quantity proportional to $v_{i, j}=d_{i, j} / \lambda_{i, j}$. Integrating Equation (3.9) with respect to $\eta$ and making use of Equation (3.5), one obtains,

$$
\begin{gathered}
\bar{F}_{p p(i, j)}^{d i v}\left(t, \mathbf{x}_{1}, \boldsymbol{\xi}_{1}, \mathbf{v}^{(i, j)}\right)=\left(1-\frac{\lambda_{l, j}}{2} \frac{m_{j}}{\left(m_{i}+m_{j}\right)} \frac{\partial}{\partial x_{1 \eta}}+\ldots\right) \bar{F}_{p(i, j)}^{d i v}\left(t, \mathbf{x}_{1}, \mathbf{G}, \mathbf{v}^{(i, j)}\right)= \\
=\left(1-\frac{\tau_{l, j}}{2} \frac{m_{j}}{\left(m_{i}+m_{j}\right)} \mathbf{v}^{(i, j)} \frac{\partial}{\partial \mathbf{x}_{1}}+\ldots\right) \bar{F}_{p(i, j)}^{d i v}\left(t, \mathbf{x}_{1}, \mathbf{G}, \mathbf{v}^{(i, j)}\right)
\end{gathered}
$$

Relationships (3.11) can be derived similarly,

$$
\begin{gathered}
\bar{F}_{p p(i, j)}^{a p p}\left(t, \mathbf{x}_{1}, \boldsymbol{\xi}_{1}, \mathbf{v}^{(i, j)}\right)=\left(1+\frac{\tau_{l, j}}{2} \frac{m_{j}}{\left(m_{i}+m_{j}\right)} \mathbf{v}^{(i, j)} \frac{\partial}{\partial \mathbf{x}_{1}}+\ldots\right) \bar{F}_{p(i, j)}^{a p p}\left(t, \mathbf{x}_{1}, \mathbf{G}, \mathbf{v}^{(i, j)}\right) \\
\bar{F}_{p(i, j)}^{d i p p}\left(t, \mathbf{x}, \mathbf{G}, \mathbf{v}^{(i, j)}\right)=\left(1 \pm \frac{\tau_{l, j}}{2} \frac{m_{j}}{\left(m_{i}+m_{j}\right)} \mathbf{v}^{(i, j)} \frac{\partial}{\partial \mathbf{x}}+\ldots\right) \bar{F}_{p p(i, j)}^{d i v}\left(t, \mathbf{x}, \boldsymbol{\xi}_{1}, \mathbf{v}^{(i, j)}\right)
\end{gathered}
$$

Now let us derive correlations between the $\overline{\tilde{F}}_{2(i, j)}^{p( \pm)}$ and $\overline{\tilde{\tilde{F}}}_{2(i, j)}^{p( \pm)}$ functions at extreme points $\eta= \pm d_{i, j}$ and $\eta= \pm \lambda_{i, j}$. These correlations can be derived in much the same way as Equations (3.10) and (3.11),

$$
\begin{gathered}
\overline{\tilde{F}}_{2(i, j)}^{p( \pm)}\left(t, \mathbf{x}, \mathbf{G}, \mathbf{v}^{(i, j)}, b, \varepsilon, \eta= \pm d_{i, j}\right)=\overline{\tilde{\tilde{F}}}_{2(i, j)}^{p( \pm)}\left(t, \mathbf{x}, \boldsymbol{\xi}_{1}, \mathbf{v}^{(i, j)}, b, \varepsilon, \eta= \pm d_{i, j}\right) \\
\overline{\tilde{F}}_{2(i, j)}^{p( \pm)}\left(t, \mathbf{x}, \mathbf{G}, \mathbf{v}^{(i, j)}, b, \varepsilon, \eta= \pm \lambda_{i, j}\right)==\left(1 \pm \tau_{i, j} \frac{m_{j}}{\left(m_{i}+m_{j}\right)} \mathbf{v}^{(i, j)} \frac{\partial}{\partial \mathbf{x}}+\ldots\right) \overline{\tilde{\tilde{F}}}_{2(i, j)}^{p( \pm)}\left(t, \mathbf{x}, \boldsymbol{\xi}_{1}, \mathbf{v}^{(i, j)}, b, \varepsilon, \eta= \pm \lambda_{i, j}\right)
\end{gathered}
$$


In the hydrodynamic $l_{h}$ - stage of description, the characteristic scale of variation of space averaged distribution functions is the characteristic flow dimension $L_{h} \gg \lambda$. Then, the high order expansion terms omitted from Equations (3.9)-(3.12) are of the order of $\mathrm{O}\left(K n^{2}\right), K n=\lambda / L_{h}$, relative to the first retained terms; thus, omitted terms corresponds to the Barnett accuracy of description (Ferziger \& Kaper, 1972).

\section{Equations for Pair Distribution Functions and Paired-Particles Distribution Functions}

Let us specify the collision cylinder $C_{\max }^{+}: 0 \leq b \leq d^{\max }, 0 \leq \varepsilon \leq 2 \pi, d^{\min } \leq \eta \leq \lambda^{\max }$. Here, $d^{\max }$ and $\lambda^{\max }$ are the maximum meanings of both the range of interaction potential and the characteristic free path length among all $i, j$-pairs, $i, j=1, \ldots, L, d^{\min }$ is the minimum meaning of the range of interaction potential. Let us specify the collision cylinder $C_{\max }^{-}: 0 \leq b \leq d^{\max }, 0 \leq \varepsilon \leq 2 \pi,-\left(\lambda^{\max }\right) \leq \eta \leq-\left(d^{\min }\right)$. Integrating Equation (2.17) with respect to $\rho$ over cylindrical volume $C_{\max }^{+}$yields,

$$
\begin{gathered}
\sum_{j=1}^{L}\left(\frac{\partial}{\partial t}+\mathbf{G} \frac{\partial}{\partial \mathbf{x}}\right) N_{i} \tilde{N}_{j} \int_{C_{\max }^{+}} \widehat{F}_{2(i, j)}^{p(+)}(t, \mathbf{x}, \mathbf{G}, \mathbf{v}, \boldsymbol{\rho}) d \boldsymbol{\rho}= \\
=\sum_{j=1}^{L} \int_{0}^{2 \pi} \int_{0}^{d_{i, j}} v N_{i} \tilde{N}_{j}\left[\widehat{F}_{2(i, j)}^{p(+)}\left(t, \mathbf{x}, \mathbf{G}, \mathbf{v}, \boldsymbol{\rho}_{\mathrm{v}}^{d_{i, j}^{+}}\right)-\widehat{F}_{2(i, j)}^{p(+)}\left(t, \mathbf{x}, \mathbf{G}, \mathbf{v}, \boldsymbol{\rho}_{\mathrm{v}}^{\lambda_{i, j}^{+}}\right)\right] b d b d \varepsilon\left[1+\mathrm{O}\left(v_{i, j}\right)\right]
\end{gathered}
$$

Integrating Equation (2.17) with respect to $\rho$ over cylindrical volume $C_{\max }^{-}$yields,

$$
\begin{gathered}
\sum_{j=1}^{L}\left(\frac{\partial}{\partial t}+\mathbf{G} \frac{\partial}{\partial \mathbf{x}}\right) N_{i} \tilde{N}_{j} \int_{C_{\max }^{+}} \hat{F}_{2(i, j)}^{p(-)}(t, \mathbf{x}, \mathbf{G}, \mathbf{v}, \boldsymbol{\rho}) d \mathbf{\rho}= \\
=\sum_{j=1}^{L} \int_{0}^{2 \pi} \int_{0}^{d_{i, j}} v N_{i} \tilde{N}_{j}\left[\hat{F}_{2(i, j)}^{p(-)}\left(t, \mathbf{x}, \mathbf{G}, \mathbf{v}, \boldsymbol{\rho}_{\mathrm{v}}^{\lambda_{i, j}^{-}}\right)-\hat{F}_{2(i, j)}^{p(-)}\left(t, \mathbf{x}, \mathbf{G}, \mathbf{v}, \boldsymbol{\rho}_{\mathbf{v}}^{d_{i, j}^{-}}\right)\right] b d b d \varepsilon\left[1+\mathrm{O}\left(v_{i, j}\right)\right]
\end{gathered}
$$

In Equations (4.1) and (4.2), $\boldsymbol{\rho}_{\mathrm{v}}^{\lambda_{\mathrm{i}, j}^{ \pm}}=\left(b, \varepsilon, \pm \lambda_{i, j}\right), \boldsymbol{\rho}_{\mathrm{v}}^{d_{i, j}^{ \pm}}=\left(b, \varepsilon, \pm d_{i, j}\right)$, Figure 2. Making use Equation (2.6), let us recast Equations $(4.1)$ and $(4.2)$ in terms of the $\tilde{F}_{(i, j) d^{ \pm}}$two-particle distributions $\tilde{F}_{2(i, j)}^{p( \pm)}\left(t, \mathbf{x}, \mathbf{G}, \mathbf{v}^{(i, j)}, \boldsymbol{\rho}^{(i, j)}\right)$, $\tilde{F}_{2(i, j)}^{p( \pm)}\left(t, \mathbf{x}, \mathbf{G}, \mathbf{v}^{(i, j)}, \boldsymbol{\rho}_{\mathbf{v}^{(i, j)}}^{(i, j) d_{i, j}^{ \pm}}\right)$, and $\tilde{F}_{2(i, j)}^{p( \pm)}\left(t, \mathbf{x}, \mathbf{G}, \mathbf{v}^{(i, j)}, \boldsymbol{\rho}_{\mathbf{v}^{(i, j)}}^{(i, j) \lambda_{i, j}^{ \pm}}\right)$,

$$
\begin{gathered}
\sum_{j=1}^{L}\left(\frac{\partial}{\partial t}+\mathbf{G} \frac{\partial}{\partial \mathbf{x}}\right) N_{i} \tilde{N}_{j} F_{p(i, j)}^{d i v}\left(t, \mathbf{x}, \mathbf{G}, \mathbf{v}^{(i, j)}\right) D_{[i, j]}=\sum_{j=1}^{L} \int_{0}^{2 \pi} \int_{0}^{d_{i, j}} v^{(i, j)} N_{i} \tilde{N}_{j}\left[\tilde{F}_{2(i, j)}^{p(+)}\left(t, \mathbf{x}, \mathbf{G}, \mathbf{v}^{(i, j)}, \boldsymbol{\rho}_{\mathbf{v}^{(i, j)}}^{(i, j) d_{i, j}^{+}}\right)-\right. \\
\left.-\tilde{F}_{2(i, j)}^{p(+)}\left(t, \mathbf{x}, \mathbf{G}, \mathbf{v}^{(i, j)}, \boldsymbol{\rho}_{\mathbf{v}^{(i, j)}}^{(i, j) \lambda_{i, j}^{+}}\right)\right] D_{[i, j]} b d b d \varepsilon\left[1+\mathrm{O}\left(v_{i, j}\right)\right] \\
\sum_{j=1}^{L}\left(\frac{\partial}{\partial t}+\mathbf{G} \frac{\partial}{\partial \mathbf{x}}\right) N_{i} \tilde{N}_{j} F_{p(i, j)}^{a p p}\left(t, \mathbf{x}, \mathbf{G}, \mathbf{v}^{(i, j)}\right) D_{[i, j]}=\sum_{j=1}^{L} \int_{0}^{2 \pi d_{i, j}} \int_{0}^{(i, j)} N_{i} \tilde{N}_{j}\left[\tilde{F}_{2(i, j)}^{p(-)}\left(t, \mathbf{x}, \mathbf{G}, \mathbf{v}^{(i, j)}, \boldsymbol{\rho}_{\mathbf{v}^{(i, j)}}^{(i, j) \lambda_{i, j}^{-}}\right)-\right. \\
\left.-\tilde{F}_{2(i, j)}^{p(-)}\left(t, \mathbf{x}, \mathbf{G}, \mathbf{v}^{(i, j)}, \boldsymbol{\rho}_{\mathbf{v}^{(i, j)}}^{(i, j) d_{i, j}^{-}}\right)\right] D_{[i, j]} b d b d \varepsilon\left[1+\mathrm{O}\left(v_{i, j}\right)\right] \\
D_{[i, j]}=\frac{\partial\left(\mathbf{v}^{(i, j)}\right)}{\partial(\mathbf{v})}=\left(\frac{m_{i}+m_{j}}{2 m_{j}}\right)^{3}
\end{gathered}
$$

In Equations (4.3) and (4,4), $D_{[i, j]}$ is jakobian of the transformation (2.5). Equations (4.1) and (4.2) were derived for a pair in a gas of identical structureless particles (Lebed 2015a). Transformations of these equations by Lebed (2015a) remain true for a pair of structureless particles of $i$ - and $j$-quality in a gas mixture $i, j=1, \ldots, L$. The $\tilde{F}_{2(i, j)}^{p}\left(t, \mathbf{x}, \mathbf{G}, \mathbf{v}^{(i, j)}, \boldsymbol{\rho}_{\mathbf{v}^{(i, j)}}^{(i, j)}\right)$ function in the first term on the right hand side of Equation $(4.3)$ corresponds to a pair of particles $i$ and $j$, which leave the interaction domain $C_{i, j}^{0}\left(\boldsymbol{\rho}_{(i, j) d^{+}}^{(i, j)}=\boldsymbol{\rho}_{\mathbf{v}^{(i, j)}}^{(i, j) d_{i, j}^{+}}\right)$at relative velocity $\mathbf{v}^{(i, j)}$. Making use Equation (2.12), let us express the $\bar{F}_{2(i, j)}^{p(+)}\left(t, \mathbf{x}, \mathbf{G}, \mathbf{v}^{(i, j)}, \boldsymbol{\rho}_{\mathbf{v}^{(i, j)}}^{(i, j) d_{i, j}^{+}}\right)$function in terms of the two-particle distribution function at the entrance to the domain $C_{i, j}^{0}$. With due regard for Equation (2.6) in Lebed (2015a), one obtains, 


$$
\tilde{F}_{2(i, j)}^{p(+)}\left(t, \mathbf{x}, \mathbf{G}, \mathbf{v}^{(i, j)}, \boldsymbol{\rho}_{\mathbf{v}^{(i, j)}}^{(i, j) d_{i, j}^{+}}\right)=\tilde{F}_{2(i, j)}^{p(-)}\left(t, \mathbf{x}, \mathbf{G}, \mathbf{v}^{(i, j)}, \boldsymbol{\rho}_{\mathbf{v}^{(i, j)}}^{(i, j) d, j}\right)\left[1+\mathrm{O}\left(v_{i, j}\right)\right]
$$

If particles $i$ and $j$ enter the domain $C_{i, j}^{0}$ at relative velocity $\mathbf{v}^{(i, j)}=\mathbf{v}^{(i, j)}\left(b, \mathcal{E}, \mathbf{v}^{(i, j)}, d_{i, j}, \mu_{i, j}\right)$, then they have velocity $\mathbf{v}^{(i, j)}$ at the exit of the $C_{i, j}^{0}$. Substitute Equation (4.5) in Equation (4.iv) and express the $\tilde{F}_{2(i, j)}^{p( \pm)}$ functions at extreme points $\eta=+\lambda_{i, j}$ and $\eta=-d_{i, j}$ in terms of pair functions $F_{p(i, j)}^{a p p}$ (3.1). From Equations (3.3)-(3.6) one obtains through repeated substitutions,

$$
\begin{gathered}
\sum_{j=1}^{L}\left(\frac{\partial}{\partial t}+\mathbf{G} \frac{\partial}{\partial \mathbf{x}}\right) N_{i} \tilde{N}_{j} F_{p(i, j)}^{d i v}\left(t, \mathbf{x}, \mathbf{G}, \mathbf{v}^{(i, j)}\right) D_{[i, j]}= \\
=\sum_{j=1}^{L} \int_{0}^{2 \pi} \int_{0}^{d_{i, j}} \frac{N_{i} \tilde{N}_{j}}{\tau_{i, j}}\left[F_{p(i, j)}^{a p p}\left(t, \mathbf{x}, \mathbf{G}, \mathbf{v}^{(i, j)}, b, \varepsilon\right)-F_{p(i, j)}^{d i v}\left(t, \mathbf{x}, \mathbf{G}, \mathbf{v}^{(i, j)}, b, \varepsilon\right)\right] D_{[i, j]} b d b d \varepsilon\left[1+\mathrm{O}\left(v_{i, j}\right)\right]
\end{gathered}
$$

The first term on the right hand side of Equation (4.6) contains information, which is excessive for the kinetic stage of gas description. This is information about mutual disposition of particles in the pair contained in the $F_{p(i, j)}^{a p p}$ dependence on $b$ and $\varepsilon$. According to Lebed (2014c), in order to proceed to the kinetic stage, we replace this function by its average value,

$$
F_{p}^{a p p}\left(t, \mathbf{x}, \mathbf{G}, \mathbf{v}^{(i, j)}, b, \varepsilon\right) \Rightarrow \frac{1}{\sigma_{i, j}} F_{p}^{a p p}\left(t, \mathbf{x}, \mathbf{G}, \mathbf{v}^{(i, j)}\right) \quad \sigma_{i, j}=\pi d_{i, j}^{2}
$$

here,

$$
F_{p}^{a p p}\left(t, \mathbf{x}, \mathbf{G}, \mathbf{v}^{(i, j)}\right)=\left.\int_{0}^{2 \pi} \int_{0}^{d_{i, j}} F_{p}^{a p p}\left(t, \mathbf{x}, \mathbf{G}, \mathbf{v}^{(i, j)}, b, \varepsilon\right)\right|_{\mathbf{v}^{(i, j)}} b d b d \varepsilon
$$

The replacement (4.7), being substituted in Equation (4.6), makes this equation irreversible. The physical meaning of operation (4.7) was disclosed by Lebed (2014c). Introduce the operation (3.7) of space averaging of functions in Equation (4.6). Let us specify pair functions $f_{p(i, j)}^{d i v}$ and $f_{p(i, j)}^{a p p}$,

$$
f_{p(i, j)}^{d i v}\left(t, \mathbf{x}, \mathbf{G}, \mathbf{v}^{(i, j)}\right)=N_{i} \tilde{N}_{j} \bar{F}_{p(i, j)}^{d i v p}\left(t, \mathbf{x}, \mathbf{G}, \mathbf{v}^{(i, j)}\right)
$$

The $f_{p(i, j)}^{d i v}\left(t, \mathbf{x}, \mathbf{G}, \mathbf{v}^{(i, j)}\right)$ pair distribution function has a meaning of a probable number of particles of $i$ - quality flying apart particles of $j$-quality after a collision with each other with the centers of mass of which are concentrated in a unit volume element near $\mathbf{x}$ and velocities, in a unit interval near $\mathbf{G}, \mathbf{v}^{(i, j)}$ at time $t$. The $f_{p(i, j)}^{a p p}\left(t, \mathbf{x}, \mathbf{G}, \mathbf{v}^{(i, j)}\right)$ pair distribution function has a meaning of a probable number of particles of $i$ - quality approaching particles of $j$-quality before a collision with each other with the centers of mass of which are concentrated in a unit volume element near $\mathbf{x}$ and velocities, in a unit interval near $\mathbf{G}, \mathbf{v}^{(i, j)}$ at time $t$.

Let us dwell on a case where $\tau$ is assumed to be common for all the pairs of $i-$ and $j$-particles without exception, $i, j=1, \ldots, L$. In the case of common $\tau$, let us recast Equation (4.6) in terms of pair functions (4.8),

$$
\begin{gathered}
\sum_{j=1}^{L}\left(\frac{\partial}{\partial t}+\mathbf{G} \frac{\partial}{\partial \mathbf{x}}\right) f_{p(i, j)}^{d i v}\left(t, \mathbf{x}, \mathbf{G}, \mathbf{v}^{(i, j)}\right) D_{[i, j]}= \\
=\sum_{j=1}^{L} \int_{0}^{2 \pi} \int_{0}^{d_{i, j}} \frac{f_{p(i)}^{a p p}\left(t, \mathbf{x}, \mathbf{G}, \mathbf{v}^{(i, j)}\right)-f_{p(i)}^{d i v}\left(t, \mathbf{x}, \mathbf{G}, \mathbf{v}^{(i, j)}\right)}{\tau} D_{[i, j]} \frac{b d b d \varepsilon}{\sigma_{i, j}}
\end{gathered}
$$

The terms omitted from Equation (4.9) are of the order of $\mathrm{O}\left(v_{i, j}\right)$. Let $\theta, \varphi$, and $v^{(i, j)}$ be the spherical coordinates of the vector $\mathbf{v}^{(i, j)}$. Integrating Equation (4.9) with respect to the spherical angles yields,

$$
\left(\frac{\partial}{\partial t}+\mathbf{G} \frac{\partial}{\partial \mathbf{x}}\right) f_{p(i)}^{d i v}(t, \mathbf{x}, \mathbf{G}, v)=\frac{f_{p(i)}^{a p p}(t, \mathbf{x}, \mathbf{G}, v)-f_{p(i)}^{d i v}(t, \mathbf{x}, \mathbf{G}, v)}{\tau}
$$

here,

$$
\begin{gathered}
f_{p(i, j)}^{d i v}\left(t, \mathbf{x}, \mathbf{G}, v^{(i, j)}\right)=\int f_{p(i, j)}^{d i v}\left(t, \mathbf{x}, \mathbf{G}, \mathbf{v}^{(i, j)}\right) \sin \theta d \theta d \varphi \\
f_{p(i)}^{a p p}(t, \mathbf{x}, \mathbf{G}, v)=\sum_{j=1}^{L} f_{p(i, j)}^{d i v}\left(t, \mathbf{x}, \mathbf{G}, v^{(i, j)}\right) D_{[i, j]}
\end{gathered}
$$


The $\tilde{F}_{2(i, j)}^{p(-)}\left(t, \mathbf{x}, \mathbf{G}, \mathbf{v}^{(i, j)}, \boldsymbol{\rho}_{\left.\mathbf{v}^{(i, j, j}\right)}^{d_{i, j}^{-}}\right)$function in the second term on the right hand side of Equation (4.4) corresponds to a pair of particles $i$ and $j$, which enter the interaction domain $C_{i, j}^{0}\left(\boldsymbol{\rho}_{d^{-}}^{(i, j)}=\boldsymbol{\rho}_{\mathbf{v}^{i, j, j}}^{d^{i, j}}\right)$ at relative velocity $\mathbf{v}^{(i, j)}$. Making use Equation (2.12), let us express the $\tilde{F}_{2(i, j)}^{p(-)}\left(t, \mathbf{x}, \mathbf{G}, \mathbf{v}^{(i, j)}, \boldsymbol{\rho}_{\mathbf{v}^{(i, j)}}^{d_{i, j}}\right)$ function in terms of the two-particle distribution function at the exit to the domain $C_{i, j}^{0}$. With due regard for Equation (3.20) in Lebed (2015a), one obtains,

$$
\tilde{F}_{2(i, j)}^{p(-)}\left(t, \mathbf{x}, \mathbf{G}, \mathbf{v}^{(i, j)}, \boldsymbol{\rho}_{\mathbf{v}^{(i, j)}}^{d_{i, j}^{-}}\right)=\tilde{F}_{2(i, j)}^{p(+)}\left(t, \mathbf{x}, \mathbf{G}, \mathbf{v}^{\mathbf{n}(i, j)}, \boldsymbol{\rho}_{\mathbf{v}^{(i, j, j}}^{d_{i, j}^{+}}\right)\left[1+\mathrm{O}\left(v_{i, j}\right)\right]
$$

If particles $i$ and $j$ enter the $C_{i, j}^{0}$ at relative velocity $\mathbf{v}^{(i, j)}$, then they have velocity $\mathbf{v}^{\prime \prime}=\mathbf{v}^{\text {" }(i, j)}\left(b, \mathcal{E}, \mathbf{v}^{(i, j)}, d_{i, j}, \mu_{i, j}\right)$ at the exit of the $C_{i, j}^{0}$. Substitute Equation (4.12) in Equation (4iv 4$)$ and express the $\tilde{F}_{2(i, j)}^{p( \pm)}$ functions at extreme points $\eta=-\lambda_{i, j}$ and $\eta=+d_{i, j}$ in terms of pair functions $F_{p(i, j)}^{a p p}$ (3.1). From Equations (3.3)-(3.6) one obtains through repeated substitutions,

$$
\begin{gathered}
\sum_{j=1}^{L}\left(\frac{\partial}{\partial t}+\mathbf{G} \frac{\partial}{\partial \mathbf{x}}\right) N_{i} \tilde{N}_{j} F_{p(i, j)}^{a p p}\left(t, \mathbf{x}, \mathbf{G}, \mathbf{v}^{(i, j)}\right) D_{[i, j]}= \\
=\sum_{j=1}^{L} \int_{0}^{2 \pi} \int_{0}^{d_{i, j}} \frac{N_{i} \tilde{N}_{j}}{\tau}\left[F_{p(i, j)}^{a p p}\left(t, \mathbf{x}, \mathbf{G}, \mathbf{v}^{(i, j)}, b, \varepsilon\right)-F_{p(i, j)}^{d i v}\left(t, \mathbf{x}, \mathbf{G}, \mathbf{v}^{\text {(i, }}{ }^{(i, j)}, b, \varepsilon\right)\right] D_{[i, j]} b d b d \varepsilon
\end{gathered}
$$

The terms omitted from Equation (4.13) are of the order of $\mathrm{O}\left(v_{i, j}\right)$. Without performing the irreversible replacement in Equation (4.13), we integrate this equation with respect to the spherical angles of relative velocity vector. Then, averaging Equation (4.13) within the $S$ region near point $\mathbf{x}$ (3.7), and using definitions (4.8), we arrive at the equation:

$$
\left(\frac{\partial}{\partial t}+\mathbf{G} \frac{\partial}{\partial \mathbf{x}}\right) f_{p(i)}^{a p p}(t, \mathbf{x}, \mathbf{G}, v)=\frac{f_{p(i)}^{a p p}(t, \mathbf{x}, \mathbf{G}, v)-f_{p(i)}^{d i v}(t, \mathbf{x}, \mathbf{G}, v)}{\tau}
$$

The solution to the closed set of Equations (4.10) and (4.14) discovers the basic property of the pair distribution functions (Lebed, 1990; 1995). It shows that the distribution functions $f_{p(i)}^{d i v}(t, \mathbf{x}, \mathbf{G}, v)$ and $f_{p(i)}^{a p p}(t, \mathbf{x}, \mathbf{G}, v)$ do not change with time along the trajectory of the center of mass of a pair:

$$
\begin{gathered}
\left(\frac{\partial}{\partial t}+\mathbf{G} \frac{\partial}{\partial \mathbf{x}}\right) f_{p(i)}(t, \mathbf{x}, \mathbf{G}, v)=0 \\
f_{p(i)}^{d i v}(t, \mathbf{x}, \mathbf{G}, v)=f_{p(i)}^{a p p}(t, \mathbf{x}, \mathbf{G}, v)=f_{p(i)}(t, \mathbf{x}, \mathbf{G}, v)
\end{gathered}
$$

Let us multiply Equation (4.15) by $m_{i} v^{2} / 4$,

$$
\left(\frac{\partial}{\partial t}+\mathbf{G} \frac{\partial}{\partial \mathbf{x}}\right) \frac{m_{i} v^{2}}{4} f_{p(i)}(t, \mathbf{x}, \mathbf{G}, v)=0
$$

Here, with due regard for relationships (2.5) and (4.11), one obtains,

$$
\frac{m_{i} v^{2}}{4} f_{p(i)}(t, \mathbf{x}, \mathbf{G}, v)=\sum_{j=1}^{L} \int \frac{m_{i} m_{j}^{2}}{\left(m_{i}+m_{j}\right)^{2}}\left(v^{(i, j)}\right)^{2} f_{p(i, j)}^{d i v}\left(t, \mathbf{x}, \mathbf{G}, \mathbf{v}^{(i, j)}\right) D_{[i, j]} \sin \theta d \theta d \varphi
$$

The basic property (4.15) means that particle collisions cannot influence the formation of hydrodynamic values constructed on the pair properties of $\varphi_{\alpha_{1} \ldots \alpha_{l}}^{1(l)}(\mathbf{G}), l=0,1, \ldots$, and $m_{j}^{2}\left(v^{(i, j)}\right)^{2} /\left(m_{i}+m_{j}\right)^{2}$. In other words, particle collisions cannot tune the distributions of all these hydrodynamic values to distributions of some other hydrodynamic values. That is, the set of hydrodynamic values constructed on the properties of $\varphi_{\alpha_{1} \ldots \alpha_{l}}^{1(l)}(\mathbf{G})$, $l=0,1, \ldots$, and $\varphi^{2(k)}\left(m_{j}^{2}\left(v^{(i, j)}\right)^{2} /\left(m_{i}+m_{j}\right)^{2}\right), k=0,1, \ldots$,

$$
M_{\alpha_{1} \ldots a_{l}(i)}^{(l, k)}(t, \mathbf{x})=\sum_{j=1}^{L} \int \varphi_{\alpha_{1} \ldots \alpha_{l}}^{1(l)}(\mathbf{G}) \varphi^{2(k)}\left(m_{j}^{2}\left(v^{(i, j)}\right)^{2} /\left(m_{i}+m_{j}\right)^{2}\right) f_{p(i, j)}^{d i v p}\left(t, \mathbf{x}, \mathbf{G}, \mathbf{v}^{(i, j)}\right) d \mathbf{G} d \mathbf{v}^{(i, j)}
$$

is a set of principle hydrodynamic values.

Integrating Equations (2.16) with respect to $\boldsymbol{\rho}$ over cylindrical volumes $C_{\max }^{+}$and $C_{\max }^{-}$yields, 


$$
\begin{aligned}
& \sum_{j=1}^{L}\left(\frac{\partial}{\partial t}+\xi_{1} \frac{\partial}{\partial \mathbf{x}_{1}}\right) N_{i} \tilde{N}_{j} \int_{C_{\max }^{+}} \widehat{\hat{F}}_{2(i, j)}^{p(+)}\left(t, \mathbf{x}_{1}, \xi_{1}, \mathbf{v}, \boldsymbol{\rho}\right) d \mathbf{\rho}= \\
& =\sum_{j=1}^{L} \int_{0}^{2 \pi} \int_{0}^{d_{i, j}} v N_{i} \tilde{N}_{j}\left[\widehat{\bar{F}}_{2(i, j)}^{p(-)}\left(t, \mathbf{x}_{1}, \boldsymbol{\xi}_{1}, \mathbf{v}, \boldsymbol{\rho}_{\mathbf{v}}^{d_{i, j}^{+}}\right)-\widehat{F}_{2(i, j)}^{p(-)}\left(t, \mathbf{x}_{1}, \boldsymbol{\xi}_{1}, \mathbf{v}, \boldsymbol{\rho}_{\mathbf{v}}^{\lambda_{i, j}^{+}}\right)\right] b d b d \varepsilon\left[1+\mathrm{O}\left(v_{i, j}\right)\right] \\
& \sum_{j=1}^{L}\left(\frac{\partial}{\partial t}+\xi_{1} \frac{\partial}{\partial \mathbf{x}_{1}}\right) N_{i} \tilde{N}_{j} \int_{C_{\max }^{+}} \widehat{\bar{F}}_{2(i, j)}^{p(-)}\left(t, \mathbf{x}_{1}, \xi_{1}, \mathbf{v}, \boldsymbol{\rho}\right) d \mathbf{\rho}= \\
& =\sum_{j=1}^{L} \int_{0}^{2 \pi} \int_{0}^{d_{i, j}} v N_{i} \tilde{N}_{j}\left[\widehat{\bar{F}}_{2(i, j)}^{p(-)}\left(t, \mathbf{x}_{1}, \xi_{1}, \mathbf{v}, \boldsymbol{\rho}_{\mathbf{v}}^{\lambda_{i, j}^{-}}\right)-\widehat{\bar{F}}_{2(i, j)}^{p(-)}\left(t, \mathbf{x}_{1}, \xi_{1}, \mathbf{v}, \boldsymbol{\rho}_{\mathbf{v}}^{d_{i, j}^{-}}\right)\right] b d b d \varepsilon\left[1+\mathrm{O}\left(v_{i, j}\right)\right]
\end{aligned}
$$

Making use Equation (2.6), let us recast Equations (4.17) and (4.18) in terms of the two-particle distributions $\tilde{\tilde{F}}_{2(i, j)}^{p}\left(t, \mathbf{x}_{1}, \xi_{1}, \mathbf{v}^{(i, j)}, \boldsymbol{\rho}^{(i, j)}\right), \tilde{F}_{2(i, j)}^{p}\left(t, \mathbf{x}_{1}, \xi_{1}, \mathbf{v}^{(i, j)}, \boldsymbol{\rho}_{\mathbf{v}^{(i, j)}}^{(i, j) d_{i, j}^{ \pm}}\right)$and $\tilde{F}_{2(i, j)}^{p}\left(t, \mathbf{x}_{1}, \xi_{1}, \mathbf{v}^{(i, j)}, \boldsymbol{\rho}_{\mathbf{v}^{(i, j)}}^{(i, j) \lambda_{i, j}^{t}}\right)$,

$$
\begin{aligned}
& \sum_{j=1}^{L}\left(\frac{\partial}{\partial t}+\xi_{1} \frac{\partial}{\partial \mathbf{x}_{1}}\right) N_{i} \tilde{N}_{j} F_{p p(i, j)}^{d i v}\left(t, \mathbf{x}_{1}, \boldsymbol{\xi}_{1}, \mathbf{v}^{(i, j)}\right) D_{[i, j]}= \\
= & \sum_{j=1}^{L} \int_{0}^{2 \pi} \int_{0}^{d_{i, j}} v^{(i, j)} N_{i} \tilde{N}_{j}\left[\tilde{F}_{2(i, j)}^{p(+)}\left(t, \mathbf{x}_{1}, \xi_{1}, \mathbf{v}^{(i, j)}, \boldsymbol{\rho}_{\mathbf{v}^{(i, j)}}^{(i, j) d_{i, j}^{+}}\right)-\right. \\
- & \left.\tilde{F}_{2(i, j)}^{p(+)}\left(t, \mathbf{x}_{1}, \boldsymbol{\xi}_{1}, \mathbf{v}^{(i, j)}, \boldsymbol{\rho}_{\mathbf{v}^{(i, j)}}^{(i, j) \lambda_{i, j}^{+}}\right)\right] D_{[i, j]} b d b d \varepsilon\left[1+\mathrm{O}\left(v_{i, j}\right)\right] \\
& \sum_{j=1}^{L}\left(\frac{\partial}{\partial t}+\xi_{1} \frac{\partial}{\partial \mathbf{x}_{1}}\right) N_{i} \tilde{N}_{j} F_{p p(i, j)}^{a p p}\left(t, \mathbf{x}_{1}, \boldsymbol{\xi}_{1}, \mathbf{v}^{(i, j)}\right) D_{[i, j]}= \\
= & \sum_{j=1}^{L} \int_{0}^{2 \pi} \int_{0}^{d_{i, j}} v^{(i, j)} N_{i} \tilde{N}_{j}\left[\tilde{F}_{2(i, j)}^{p(-)}\left(t, \mathbf{x}_{1}, \boldsymbol{\xi}_{1}, \mathbf{v}^{(i, j)}, \boldsymbol{\rho}_{\left.\mathbf{v}^{(i, j)}\right)}^{(i, j) \lambda_{i, j}^{-}}\right)-\right. \\
- & \left.\tilde{F}_{2(i, j)}^{p(-)}\left(t, \mathbf{x}_{1}, \boldsymbol{\xi}_{1}, \mathbf{v}^{(i, j)}, \boldsymbol{\rho}_{\mathbf{v}^{(i, j)}}^{(i, j) d_{i, j}^{-}}\right)\right] D_{[i, j]} b d b d \varepsilon\left[1+\mathrm{O}\left(v_{i, j}\right)\right]
\end{aligned}
$$

In accordance with ideas of the kinetic theory of gases on a free path, in rarefied gas every particle at every instant finds itself in a dual position, namely, a particle was at once flying away from its latest collision partner and approaching its next collision partner, Figure 1. These ideas were reflected in Equations (3) and (7) in Lebed (1995). After transformation on Equations (3) and (7) from Lebed (1995) for a gas mixture they assume the form:

$$
\begin{aligned}
& \sum_{j=1}^{L} \iint_{0}^{2 \pi} \int_{0}^{d_{i, j}} v^{(i, j)} N_{i} \tilde{N}_{j} \overline{\tilde{F}}_{2(i, j)}^{p(+)}\left(t, \mathbf{x}_{1}, \boldsymbol{\xi}_{1}, \mathbf{v}^{(i, j)}, \boldsymbol{\rho}_{\mathbf{v}^{(i, j)}}^{(i, j) \lambda_{i, j}^{+}}\right) b d b d \varepsilon d \mathbf{v}^{(i, j)}= \\
& =\sum_{j=1}^{L} \iint_{0}^{2 \pi} \int_{0}^{d_{i, j}} v^{(i, j)} N_{i} \tilde{N}_{j} \overline{\tilde{F}}_{2(i, j)}^{p(-)}\left(t, \mathbf{x}_{1}, \boldsymbol{\xi}_{1}, \mathbf{v}^{(i, j)}, \boldsymbol{\rho}_{\mathbf{v}^{(i, j)}}^{(i, j) d_{i, j}^{-}}\right) b d b d \varepsilon d \mathbf{v}^{(i, j)} \\
& \sum_{j=1}^{L} \iint_{0}^{2 \pi} \int_{0}^{d_{i, j}} v^{(i, j)} N_{i} \tilde{N}_{j} \overline{\tilde{F}}_{2(i, j)}^{p(-)}\left(t, \mathbf{x}_{1}, \boldsymbol{\xi}_{1}, \mathbf{v}^{(i, j)}, \boldsymbol{\rho}_{\mathbf{v}^{(i, j)}}^{(i, j) \lambda_{i, j}^{-}}\right) b d b d \varepsilon d \mathbf{v}^{(i, j)}= \\
& =\sum_{i=1}^{L} \iint_{0}^{2 \pi} \int_{i}^{d_{i, j}} v^{(i, j)} N_{i} \tilde{N}_{j} \overline{\tilde{F}}_{2(i, j)}^{p(+)}\left(t, \mathbf{x}_{1}, \boldsymbol{\xi}_{1}, \mathbf{v}^{(i, j)}, \boldsymbol{\rho}_{\mathbf{v}^{(i, j)}}^{(i, j) d_{i, j}^{+}}\right) b d b d \varepsilon d \mathbf{v}^{(i, j)}
\end{aligned}
$$

On the other hand, once each particle at any instant reduces from its latest collision partner and simultaneously approaches its next collision partner,

$$
\bar{F}_{1(i)}\left(t, \mathbf{x}_{1}, \xi_{1}\right)=\sum_{j=1}^{L} \int \tilde{N}_{j} \bar{F}_{p p(i, j)}^{d i v}\left(t, \mathbf{x}_{1}, \boldsymbol{\xi}_{1}, \mathbf{v}^{(i, j)}\right) d \mathbf{v}^{(i, j)}
$$




$$
\bar{F}_{1(i)}\left(t, \mathbf{x}_{1}, \boldsymbol{\xi}_{1}\right)=\sum_{j=1}^{L} \int \tilde{N}_{j} \bar{F}_{p p(i, j)}^{a p p}\left(t, \mathbf{x}_{1}, \boldsymbol{\xi}_{1}, \mathbf{v}^{(i, j)}\right) d \mathbf{v}^{(i, j)}
$$

In Equation (4.22), $F_{1(i)}\left(t, \mathbf{x}_{1}, \xi_{1}\right)$ is the one-particle distribution function subject to the first equation of the BBGKY hierarchy (Liboff, 1969). Let us average Equations (4.19) and (4.20) within the $S$ region near point $\mathbf{x}_{1}$ and integrate with respect to velocity $\mathbf{v}$. With due regard for Equations (4.21), Equations (4.19) and (4.20) become,

$$
\begin{aligned}
\sum_{j=1}^{L} \int\left(\frac{\partial}{\partial t}+\xi_{1}\right. & \left.\frac{\partial}{\partial \mathbf{x}_{1}}\right) N_{i} \tilde{N}_{j} \bar{F}_{p p(i, j)}^{d i v}\left(t, \mathbf{x}_{1}, \xi_{1}, \mathbf{v}^{(i, j)}\right) d \mathbf{v}^{(i, j)}=\widehat{J}_{(i)}\left(t, \mathbf{x}_{1}, \xi_{1}\right) \\
\widehat{J}_{(i)}\left(t, \mathbf{x}_{1}, \boldsymbol{\xi}_{1}\right) & =\sum_{j=1}^{L} \iint_{0}^{2 \pi} \int_{0}^{d_{i, j}} v^{(i, j)} N_{i} \tilde{N}_{j}\left[\overline{\tilde{F}}_{2(i, j)}^{p(+)}\left(t, \mathbf{x}_{1}, \xi_{1}, \mathbf{v}^{(i, j)}, \boldsymbol{\rho}_{\mathbf{v}^{(i, j)}}^{(i, j) d_{i, j}^{+}}\right)-\right. \\
& \left.-\overline{\tilde{F}}_{2(i, j)}^{p(-)}\left(t, \mathbf{x}_{1}, \boldsymbol{\xi}_{1}, \mathbf{v}^{(i, j)}, \boldsymbol{\rho}_{\mathbf{v}^{(i, j)}}^{(i, j) d_{i, j}^{-}}\right)\right] b d b d \varepsilon d \mathbf{v}^{(i, j)}
\end{aligned}
$$

With due regard for Equation (4.22), Equation (4.23) becomes,

$$
\left(\frac{\partial}{\partial t}+\xi_{1} \frac{\partial}{\partial \mathbf{x}_{1}}\right) N_{i} \bar{F}_{1(i)}\left(t, \mathbf{x}_{1}, \xi_{1}\right)=\widehat{J}_{(i)}\left(t, \mathbf{x}_{1}, \xi_{1}\right)
$$

The terms omitted from collision integral (4.23) are of the order of $\mathrm{O}\left(v_{i, j}\right)$. Equation (4.24) was derived by Lebed (2015a) for a gas of identical structureless particles (Equations (2.15)-(2.17) in Lebed (2015a)). The derivation by Lebed (2015a) remains true for a pair of structureless particles of $i$ - and $j$-quality in a gas mixture $i, j=1, \ldots, L$. Equations (4.21a) and (4.22a), and Equations (4.21b) and (4.22b) are in essence, different representations of the same condition. Indeed, substituting Equation (4.22a) in Equation (4.23) and making use Equation (4.24) derived by Lebed (2015a), one arrives at Equation (4.21a), while the same operations on Equations (4.22b) yield Equation (4.21b).

It may happen that certain particle can not find a partner to form a pair at some instant (Lebed, 1995). In deriving Equations (3) and (7) by Lebed (1995), we disregard particles forming no pair (single particles). Thus, neglect of single particles introduced no distortions into the pattern of evolution of one-particle distribution function (4.24). The aforesaid implies that disregard of single particles fails no introduce anywhere near significant error into the model in the kinetic and hydrodynamic stages (Lebed, 1996a).

The $\tilde{\tilde{F}}_{2(i, j)}^{p(+)}\left(t, \mathbf{x}_{1}, \boldsymbol{\xi}_{1}, \mathbf{v}^{(i, j)}, \boldsymbol{\rho}_{\mathbf{v}^{(i, j)}}^{(i, j) d_{i, j}^{+}}\right)$function in the first term of collision integral $(4.23)$ corresponds to a pair of particles $i$ and $j$, which leave the interaction domain $C_{i, j}^{0}\left(\boldsymbol{\rho}^{(i, j)}=\boldsymbol{\rho}_{(i, j) d_{i, j}^{+}}\right)$at relative velocity $\mathbf{v}_{(i, j)}^{(i, j)}$. Making use relationship (2.14) and Equation (2.13), let us express the $\tilde{F}_{2(i, j)}^{p(+)}\left(t, \mathbf{x}_{1}, \boldsymbol{\xi}_{1}, \mathbf{v}^{(i, j)}, \boldsymbol{\rho}_{\mathbf{v}^{(i, j)}}^{(i, j) d_{i, j}^{+}}\right.$) function in terms of the two-particle distribution function at the entrance to the domain $C_{i, j}^{0}$. With due regard for Equation (2.6) in Lebed (2015a), one obtains,

$$
\begin{gathered}
\tilde{F}_{2(i, j)}^{p(+)}\left(t, \mathbf{x}_{1}, \boldsymbol{\xi}_{1}, \mathbf{v}^{(i, j)}, \boldsymbol{\rho}_{\mathbf{v}^{(i, j)}}^{(i, j) d_{i, j}^{+}}\right)=\breve{F}_{2(i, j)}^{p(+)}\left(t, \breve{\mathbf{x}}_{1}^{(i, j)}=\mathbf{x}_{1}, \breve{\xi}_{1}^{(i, j)}=\boldsymbol{\xi}_{1}, \breve{\mathbf{v}}^{(i, j)}=\mathbf{v}^{(i, j)}, \breve{\boldsymbol{\rho}}^{(i, j)}=\boldsymbol{\rho}_{\mathbf{v}^{(i, j)}}^{(i, j) d_{i, j}^{+}}\right)= \\
=\breve{F}_{2(i, j)}^{p(-)}\left(t, \breve{\mathbf{x}}_{1}^{(i, j)}=\mathbf{x}_{1}^{(i, j)}, \breve{\xi}_{1}^{(i, j)}=\xi_{1}^{(i, j)}, \breve{\mathbf{v}}^{(i, j)}=\mathbf{v}^{(i, j)}, \breve{\boldsymbol{\rho}}^{(i, j)}=\boldsymbol{\rho}_{\mathbf{v}^{(i, j)}}^{(i, j) d_{i, j}^{-}}\right)\left[1+\mathrm{O}\left(v_{i, j}\right)\right] \\
\mathbf{x}_{1}^{(i, j)}=\mathbf{x}_{1}-\frac{m_{j}}{m_{i}+m_{j}}\left(\boldsymbol{\rho}_{\mathbf{v}^{(i, j)}}^{(i, j) d_{i, j}^{+}}-\boldsymbol{\rho}_{\mathbf{v}^{(i, j)}}^{(i, j) d_{i, j}^{-}}\right)
\end{gathered}
$$

If particles $i$ and $j$ enter the $C_{i, j}^{0}$ at velocities $\xi_{1}^{(i, j)}=\xi_{1}^{(i, j)}\left(b, \varepsilon, \mathbf{v}^{(i, j)}, d_{i, j}, \mu_{i, j}\right)$ and $\xi_{2}^{(i, j)}=\xi_{2}^{(i, j)}\left(b, \varepsilon, \mathbf{v}^{(i, j)}, d_{i, j}, \mu_{i, j}\right), \xi_{1}^{(i, j)}-\xi_{2}^{(i, j)}=\mathbf{v}_{i, i, j)}^{(i, j)}=\mathbf{v}^{(i, j)}\left(b, \varepsilon, \mathbf{v}^{(i, j)}, d_{i, j}, \mu_{i, j}\right)$, then they have velocities $\xi_{1}$ and $\xi_{2}^{(i, j)}, \xi_{1}-\xi_{2}^{(i, j)}=\mathbf{v}^{(i, j)}$, at the exit of the $C_{i, j}^{0}$. Substitute Equation (4.25) in Equation (4.24) with collision integral (4.23), then,

$$
\begin{gathered}
\left(\frac{\partial}{\partial t}+\xi_{1} \frac{\partial}{\partial \mathbf{x}_{1}}\right) f_{1(i)}\left(t, \mathbf{x}_{1}, \xi_{1}\right)=\widehat{J}_{(i)}\left(t, \mathbf{x}_{1}, \boldsymbol{\xi}_{1}\right) \\
\widehat{J}_{(i)}\left(t, \mathbf{x}_{1}, \boldsymbol{\xi}_{1}\right)=\sum_{j=1}^{L} \widehat{J}_{(i, j)}\left(t, \mathbf{x}_{1}, \boldsymbol{\xi}_{1}\right)=
\end{gathered}
$$




$$
=\sum_{j=1}^{L} \iint_{0}^{2 \pi} \int_{0}^{d_{i, j}} v^{(i, j)} N_{i} \tilde{N}_{j}\left[\overline{\breve{F}}_{2(i, j)}^{p(+)}\left(t, \mathbf{x}_{1}, \boldsymbol{\xi}_{1}^{(i, j)}, \mathbf{v}^{(i, j)}, \boldsymbol{\rho}_{\mathbf{v}^{\mathbf{v}^{(i, j)}}}^{(i, j) d_{i, j}^{-}}\right)-\overline{\breve{F}}_{2(i, j)}^{p(-)}\left(t, \mathbf{x}_{1}, \boldsymbol{\xi}_{1}, \mathbf{v}^{(i, j)}, \boldsymbol{\rho}_{\mathbf{v}^{(i, j)}}^{(i, j) d_{i, j}^{-}}\right)\right] b d b d \varepsilon d \mathbf{v}^{(i, j)}
$$

here,

$$
f_{1(i)}\left(t, \mathbf{x}_{1}, \xi_{1}\right)=\frac{N_{i}}{l^{3}} \int_{S} F_{1(i)}\left(t, \mathbf{x}_{1}+\mathbf{a}, \xi_{1}\right) d \mathbf{a}
$$

The terms omitted from Equation (4.26) are of the order of $\mathrm{O}\left(v_{i, j}\right)$. The first term of the collision integral (4.26) contains information, which is excessive for the kinetic stage of gas description. This is information about mutual disposition of particles in the pair contained in the $\left.\bar{F}_{2(i, j)}^{p+)}\left(t, \mathbf{x}_{1}, \boldsymbol{\xi}_{1}^{(i, j)}, \mathbf{v}^{(i, j)}, \boldsymbol{\rho}_{\mathbf{v}^{(i, j)}}^{(i, j)}\right)_{i, j}^{-}\right)$dependence on $b$ and $\varepsilon$. According to Lebed (2014c), in order to proceed to the kinetic stage, we replace this function by its average value,

$$
\breve{F}_{2(i, j)}^{p(-)}\left(t, \mathbf{x}_{1}, \xi_{1}^{(i, j)}, \mathbf{v}^{(i, j)}, b, \varepsilon,-d_{i, j}\right) \Rightarrow \frac{1}{\sigma_{i, j}} \breve{F}_{2(i, j)}^{p(-)}\left(t, \mathbf{x}_{1}, \xi_{1}^{(i, j)}, \mathbf{v}^{(i, j)},-d_{i, j}\right) \sigma_{i, j}=\pi d_{i, j}^{2}
$$

here,

$$
\breve{F}_{2(i, j)}^{p(-)}\left(t, \mathbf{x}_{1}, \xi_{1}^{(i, j)}, \mathbf{v}^{(i, j)},-d_{i, j}\right)=\left.\int_{0}^{2 \pi} \int_{0}^{d_{i, j}} \breve{F}_{2(i, j)}^{p(-)}\left(t, \mathbf{x}_{1}, \boldsymbol{\xi}_{1}^{(i, j)}, \mathbf{v}^{(i, j)}, b, \varepsilon,-d_{i, j}\right)\right|_{\mathbf{v}^{(i, j)}} b d b d \varepsilon
$$

The replacement (4.27), being substituted in collision integral (4.26), makes Equation (4.26) irreversible,

$$
\begin{gathered}
\left(\frac{\partial}{\partial t}+\xi_{1} \frac{\partial}{\partial \mathbf{x}_{1}}\right) f_{1(i)}\left(t, \mathbf{x}_{1}, \xi_{1}\right)=J_{(i)}\left(t, \mathbf{x}_{1}, \xi_{1}\right) \\
J_{(i)}\left(t, \mathbf{x}_{1}, \xi_{1}\right)=\sum_{j=1}^{L} J_{(i, j)}\left(t, \mathbf{x}_{1}, \xi_{1}\right)= \\
=\sum_{j=1}^{L} \int_{0}^{2 \pi} \int_{0}^{d_{i, j}} v^{(i, j)} N_{i} \tilde{N}_{j}\left[\bar{\Xi}_{2(i, j)}^{p(-)}\left(t, \mathbf{x}_{1}, \xi_{1}^{(i, j)}, \mathbf{v}^{(i, j)},-d_{i, j}\right)-\bar{\Xi}_{2(i, j)}^{p(-)}\left(t, \mathbf{x}_{1}, \boldsymbol{\xi}_{1}, \mathbf{v}^{(i, j)},-d_{i, j}\right)\right] \frac{b d b d \varepsilon}{\sigma_{i, j}} d \mathbf{v}^{(i, j)}
\end{gathered}
$$

Let us relate one-particle distribution function $f_{1(i)}\left(t, \mathbf{x}, \boldsymbol{\xi}_{1}\right)$ to pair distribution functions $f_{p(i, j)}^{d i v}\left(t, \mathbf{x}, \mathbf{G}, \mathbf{v}^{(i, j)}\right)$. With due regard for relationships (3.10), and (3.11) one obtains,

$$
\begin{gathered}
f_{1(i)}\left(t, \mathbf{x}, \xi_{1}\right)=\sum_{j=1}^{L} \int f_{p p(i, j)}^{d i v}\left(t, \mathbf{x}, \xi_{1}, \mathbf{v}^{(i, j)}\right) d \mathbf{v}^{(i, j)}= \\
=\left.\sum_{j=1}^{l} \int\left(1 \mp \frac{\tau}{2} \frac{m_{j}}{m_{i}+m_{j}} \mathbf{v}^{(i, j)} \frac{\partial}{\partial \mathbf{x}}\right) f_{p(i, j)}^{d i v}\left(t, \mathbf{x}, \mathbf{G}=\xi_{1}-\frac{m_{j}}{\left(m_{i}+m_{j}\right)} \mathbf{v}^{(i, j)}, \mathbf{v}^{(i, j)}\right)\right|_{\xi_{1}} d \mathbf{v}^{(i, j)}
\end{gathered}
$$

here,

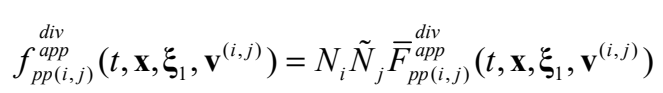

The $f_{p p(i, j)}^{d i v}\left(t, \mathbf{x}, \boldsymbol{\xi}_{1}, \mathbf{v}^{(i, j)}\right)$ paired-particle distribution function has a meaning of a probable number of particles of $i$-quality per an unit volume element near $\mathbf{x}$ that at time $t$ move with velocities from an unit interval near $\xi_{1}$ and are in the state of flying away from the particles of $j$-quality with velocities from an unit interval near $\mathbf{v}^{(i, j)}$ after a collision with each other. The $f_{p(i, j)}^{a p p}\left(t, \mathbf{x}, \xi_{1}, \mathbf{v}^{(i, j)}\right)$ paired-particle distribution function has a meaning of a probable number of particles of $i$-quality per an unit volume element near $\mathbf{x}$ that at time $t$ move with velocities from an unit interval near $\xi_{1}$ and are in the state of approaching the particles of $j$-quality with velocities from an unit interval near $\mathbf{v}^{(i, j)}$ before a collision with each other.

\section{Definition of Hydrodynamic Values}

Let us dwell on a case where $\tau$ is assumed to be common for all the pairs. From Equation (4.29) stem the following expressions for hydrodynamic values which are at a time the moments of one-particle distribution function $f_{1(i)}\left(t, \mathbf{x}, \boldsymbol{\xi}_{1}\right)$ (Ferziger \& Kaper, 1972), 


$$
\begin{gathered}
n_{(i)}(t, \mathbf{x})=\sum_{j=1}^{L} \int f_{p p(i, j)}^{d i v}\left(t, \mathbf{x}, \boldsymbol{\xi}_{1}, \mathbf{v}^{(i, j)}\right) d \xi_{1} d \mathbf{v}^{(i, j)} \\
n(t, \mathbf{x})=\sum_{i=1}^{L} n_{(i)}(t, \mathbf{x}) \rho(t, \mathbf{x})=\sum_{i=1}^{L} m_{i} n_{(i)}(t, \mathbf{x}) \\
n_{(i)}=n_{(i)}^{G} \mp \frac{\tau}{2} \sum_{j=1}^{L} \int \frac{m_{j}}{m_{i}+m_{j}} v_{k}^{(i, j)} \frac{\partial}{\partial x_{k}} f_{p(i, j)}^{d i v p}\left(t, \mathbf{x}, \mathbf{G}, \mathbf{v}^{(i, j)}\right) d \mathbf{G} d \mathbf{v}^{(i, j)}
\end{gathered}
$$

here,

$$
\begin{gathered}
n_{(i)}^{G}(t, \mathbf{x})=\sum_{j=1}^{L} n_{(i, j)}^{G}(t, \mathbf{x})=\sum_{j=1}^{L} \int f_{p(i, j)}^{d i v p}\left(t, \mathbf{x}, \mathbf{G}, \mathbf{v}^{(i, j)}\right) d \mathbf{G} d \mathbf{v}^{(i, j)}, \\
n^{G}(t, \mathbf{x})=\sum_{i=1}^{L} n_{(i)}^{G}(t, \mathbf{x}) \rho^{G}(t, \mathbf{x})=\sum_{i=1}^{L} m_{i} n_{(i)}^{G}(t, \mathbf{x})
\end{gathered}
$$

In Equations (5.1), $n=n(t, \mathbf{x})$ is the local ${ }^{i=1}$ ensity of particles number, $n_{(i)}=n_{(i)}(t, \mathbf{x})$ is the local density of number of particles of $i$-quality, $\rho=\rho(t, \mathbf{x})$ is the local mass density, $\rho_{(i)}=m_{i} n_{(i)}(t, \mathbf{x})$ is the local mass density of particles of $i$-quality.

$$
\begin{gathered}
n_{(i)}(t, \mathbf{x}) \mathbf{U}_{(i)}(t, \mathbf{x})=\sum_{j=1}^{L} \int \xi_{1} f_{p p(i, j)}^{d i v p}\left(t, \mathbf{x}, \xi_{1}, \mathbf{v}^{(i, j)}\right) d \xi_{1} d \mathbf{v}^{(i, j)} \\
\rho(t, \mathbf{x}) \mathbf{U}(t, \mathbf{x})=\sum_{i=1}^{L} m_{i} n_{(i)}(t, \mathbf{x}) \mathbf{U}_{(i)}(t, \mathbf{x}) \mathbf{V}_{(i)}(t, \mathbf{x})=\mathbf{U}_{(i)}(t, \mathbf{x})-\mathbf{U}(t, \mathbf{x}) \\
n_{(i)} \mathbf{U}_{(i)}=n_{(i)}^{G} \mathbf{U}_{(i)}^{G}+n_{(i)}^{G} \mathbf{V}_{(i)}^{v_{(i p p}^{d i v}} \mp \\
\mp \frac{\tau}{2} \sum_{j=1}^{L} \int\left[\frac{m_{j}}{m_{i}+m_{j}} \mathbf{G} v_{k}^{(i, j)} \frac{\partial}{\partial x_{k}}+\frac{m_{j}^{2}}{\left(m_{i}+m_{j}\right)^{2}} \mathbf{v}^{(i, j)} v_{k}^{(i, j)} \frac{\partial}{\partial x_{k}}\right] f_{p(i, j)}^{d i v}\left(t, \mathbf{x}, \mathbf{G}, \mathbf{v}^{(i, j)}\right) d \mathbf{G} d \mathbf{v}^{(i, j)}
\end{gathered}
$$

here,

$$
\begin{gathered}
n_{(i)}^{G}(t, \mathbf{x}) \mathbf{U}_{(i)}^{G}(t, \mathbf{x})=\sum_{j=1}^{L} n_{(i, j)}^{G}(t, \mathbf{x}) \mathbf{U}_{(i, j)}^{G}(t, \mathbf{x})=\sum_{j=1}^{L} \int \mathbf{G} f_{p(i, j)}^{d i v p}\left(t, \mathbf{x}, \mathbf{G}, \mathbf{v}^{(i, j)}\right) d \mathbf{G} d \mathbf{v}^{(i, j)} \\
n_{(i)}^{G}(t, \mathbf{x}) \mathbf{V}_{(i)}^{v^{d i p} p}(t, \mathbf{x})=\sum_{j=1}^{L} n_{(i, j)}^{G}(t, \mathbf{x}) \mathbf{V}_{(i, j)}^{v_{d i p p}^{d i v}}(t, \mathbf{x})=\sum_{j=1}^{L} \int \frac{m_{j}}{m_{i}+m_{j}} \mathbf{v}^{(i, j)} f_{p(i, j)}^{d i v}\left(t, \mathbf{x}, \mathbf{G}, \mathbf{v}^{(i, j)}\right) d \mathbf{G} d \mathbf{v}^{(i, j)} \\
\rho^{G}(t, x) \mathbf{U}^{G}(t, \mathbf{x})=\sum_{i=1}^{L} m_{i} n_{(i)}^{G}(t, \mathbf{x}) \mathbf{U}_{(i)}^{G}(t, \mathbf{x}) \mathbf{V}_{(i)}^{G}(t, \mathbf{x})=\mathbf{U}_{(i)}^{G}(t, \mathbf{x})-\mathbf{U}(t, \mathbf{x})
\end{gathered}
$$

In Equations (5.2), $\mathbf{U}=\mathbf{U}(t, \mathbf{x})$ is the hydrodynamic velocity of gas mixture, $\mathbf{U}_{(i)}=\mathbf{U}_{(i)}(t, \mathbf{x})$ is the hydrodynamic velocity of particles of $i$-quality, $\mathbf{V}_{(i)}=\mathbf{V}_{(i)}(t, \mathbf{x})$ is the diffusion velocity of particles of $i$-quality. The $\mathbf{U}$ velocity is specified in terms of average impulse.

$$
\begin{aligned}
& P_{k l}(t, \mathbf{x})=\sum_{i=1}^{L} P_{k l(i)}(t, \mathbf{x})=\sum_{i, j=1}^{L} \int m_{i}\left(\xi_{1 k}-U_{k}\right)\left(\xi_{1 l}-U_{l}\right) f_{p p(i, j)}^{d i v}\left(t, \mathbf{x}, \xi_{1}, \mathbf{v}^{(i, j)}\right) d \xi_{1} d \mathbf{v}^{(i, j)} \\
& P_{k l}=\frac{1}{2} P_{k l}^{G}+\frac{1}{2} P_{k l}^{v^{d i v p}} \mp
\end{aligned}
$$

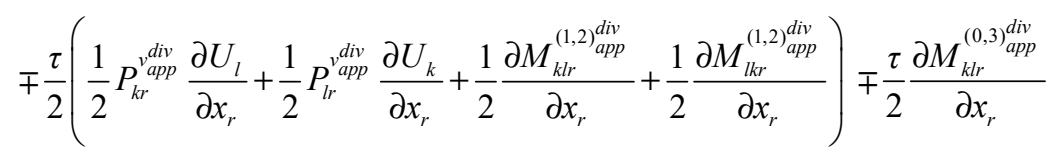

here, 


$$
\begin{gathered}
P_{k l}^{G}(t, \mathbf{x})=\sum_{i=1}^{L} P_{k l(i)}^{G}(t, \mathbf{x})==\sum_{i, j=1}^{L} P_{k l(i, j)}^{G}(t, \mathbf{x})= \\
=\sum_{i, j=1}^{L} \int 2 m_{i}\left(G_{k}-U_{k}\right)\left(G_{l}-U_{l}\right) f_{p(i, j)}^{\text {app }}\left(t, \mathbf{x}, \mathbf{G}, \mathbf{v}^{(i, j)}\right) d \mathbf{G} d \mathbf{v}^{(i, j)} \\
P_{k l}^{v^{a p p p}}(t, \mathbf{x})=\sum_{i=1}^{L} P_{k l(i)}^{v^{d a p p}}(t, \mathbf{x})=\sum_{i, j=1}^{L} P_{k l(i, j)}^{v^{d i v p}}(t, \mathbf{x})=\sum_{i, j=1}^{L} \int 2 \frac{m_{i} m_{j}^{2}}{\left(m_{i}+m_{j}\right)^{2}} v_{k}^{(i, j)} v_{l}^{(i, j)} f_{p(i, j)}^{d i v}\left(t, \mathbf{x}, \mathbf{G}, \mathbf{v}^{(i, j)}\right) d \mathbf{G} d \mathbf{v}^{(i, j)}
\end{gathered}
$$

In Equations (5.3), $P_{k l}=P_{k l}(t, \mathbf{x})$ is the stress tensor of gas mixture, tensor component $P_{k l}^{G}=P_{k l}^{G}(t, \mathbf{x})$ models the stress of gas mixture arising due to motion of centers of mass of pairs of particles, tensor component $P_{k l}^{v_{a p p}^{d i v}}=P_{k l}^{v_{a p p}^{d i v}}(t, \mathbf{x})$ allows for the stress of gas mixture generated by motion of pared particles with respect to each other, The third-order moments of pair distribution functions $M_{k l r}^{(1,2)_{a p p}^{d i v}}=M_{k l r}^{(1,2){ }_{a p p}^{d i v}}(t, \mathbf{x})$ and $M_{k l r}^{(0,3)^{d i v p}}=M_{k l r}^{(0,3)^{d i v p}}(t, \mathbf{x})$ are specified as,

$$
\begin{aligned}
& M_{k l r}^{(1,2)_{a p p}^{d i v}}(t, \mathbf{x})=\sum_{i=1}^{L} M_{k l r(i)}^{(1,2)_{a p p}^{d i v}}(t, \mathbf{x})=\sum_{i, j=1}^{L} M_{k l r(i, j)}^{(1,2)_{a p p}^{d i v}}(t, \mathbf{x}) \\
& M_{k l r}^{(1,2) \frac{d i v}{a p p}}(t, \mathbf{x})=\sum_{i, j=1}^{L} \int 2 \frac{m_{i} m_{j}^{2}}{\left(m_{i}+m_{j}\right)^{2}}\left(G_{k}-U_{k}\right) v_{l}^{(i, j)} v_{r}^{(i, j)} f_{p(i, j)}^{d i v}\left(t, \mathbf{x}, \mathbf{G}, \mathbf{v}^{(i, j)}\right) d \mathbf{G} d \mathbf{v}^{(i, j)} \\
& M_{k l r}^{(0,3)_{a p p}^{d i v}}(t, \mathbf{x})=\sum_{i=1}^{L} M_{k l r(i)}^{(0,3)_{a p p}^{d i v}}(t, \mathbf{x})=\sum_{i, j=1}^{L} M_{k l r(i, j)}^{(0,3)_{a p p}^{d i v}}(t, \mathbf{x}) \\
& M_{k l r}^{(0,3)_{a p p}^{d i v}}(t, \mathbf{x})=\sum_{i, j=1}^{L} \int \frac{m_{i} m_{j}^{3}}{\left(m_{i}+m_{j}\right)^{3}} v_{k}^{(i, j)} v_{l}^{(i, j)} v_{r}^{(i, j)} f_{p(i, j)}^{d i v}\left(t, \mathbf{x}, \mathbf{G}, \mathbf{v}^{(i, j)}\right) d \mathbf{G} d \mathbf{v}^{(i, j)}
\end{aligned}
$$

Along with $P_{k l}$, one has to determine the minor stress tensor, $p_{k l}=p_{k l}(t, \mathbf{x})$,

$$
\begin{gathered}
p_{k l}(t, \mathbf{x})=P_{k l}(t, \mathbf{x})-p(t, \mathbf{x}) \delta_{k l} p(t, \mathbf{x})=\frac{1}{3} P_{k k}(t, \mathbf{x}) \\
p_{k l}^{G}(t, \mathbf{x})=P_{k l}^{G}(t, \mathbf{x})-p^{G}(t, \mathbf{x}) \delta_{k l} p_{k l}^{v_{a p p}^{d i v}}(t, \mathbf{x})=P_{k l}^{v_{a p p}^{d i v}}(t, \mathbf{x})-p^{v}(t, \mathbf{x}) \delta_{k l} \\
p^{G}(t, \mathbf{x})=\frac{1}{3} P_{k k}^{G}(t, \mathbf{x})=\sum_{i=1}^{L} p_{(i)}^{G}(t, \mathbf{x})=\sum_{i, j=1}^{L} p_{(i, j)}^{G}(t, \mathbf{x}) \\
p^{v}(t, \mathbf{x})=\frac{1}{3} P_{k k}^{v_{a p p}^{d i v}}(t, \mathbf{x})=\sum_{i=1}^{L} p_{(i)}^{v}(t, \mathbf{x})=\sum_{i, j=1}^{L} p_{(i, j)}^{v}(t, \mathbf{x}) \\
p=\frac{1}{2} p^{G}+\frac{1}{2} p^{v} \mp \frac{\tau}{6}\left(P_{k r}^{v_{a p p}^{d i v}} \frac{\partial U_{k}}{\partial x_{r}}+\frac{\partial M_{k k r}^{(1,2)} \frac{d i v p}{\partial x_{r}}}{a p}\right) \mp \frac{\tau}{6} \frac{\partial M_{k k r}^{(0,3)}}{\partial x_{r}}
\end{gathered}
$$

In Equations (5.5), $p=p(t, \mathbf{x})$ is the pressure of gas mixture, summation is done over repetitive indices. In the kinetic theory of gases, the mean energy of thermal motion of a particle is described by a value termed as temperature $T=T(t, \mathbf{x})$. Let us define temperature $T^{G}=T^{G}(t, \mathbf{x})$ as a measure of thermal motion of centers of mass of particles and temperature $T^{v}=T^{v}(t, \mathbf{x})$, as a measure of thermal motion of paired particles with respect to each other,

$$
p(t, \mathbf{x})=n(t, \mathbf{x}) k T(t, \mathbf{x}) \quad p^{G}(t, \mathbf{x})=n(t, \mathbf{x}) k T^{G}(t, \mathbf{x}) \quad p^{v}(t, \mathbf{x})=n(t, \mathbf{x}) k T^{v}(t, \mathbf{x})
$$

Here, $k$ is the Boltzmann constant. 


$$
\begin{aligned}
& q_{l}(t, \mathbf{x})=\sum_{i=1}^{L} q_{l(i)}(t, \mathbf{x})=\sum_{i, j=1}^{L} \int \frac{m_{i}}{2}\left(\xi_{1 l}-U_{l}\right)\left(\boldsymbol{\xi}_{1}-\mathbf{U}\right)^{2} f_{p p(i, j)}^{d i v p}\left(t, \mathbf{x}, \boldsymbol{\xi}_{1}, \mathbf{v}^{(i, j)}\right) d \xi_{1} d \mathbf{v}^{(i, j)} \\
& q_{l}=\frac{1}{2} q_{l}^{G}+\frac{1}{2} q_{l}^{v}+q_{l}^{G v_{a p p}^{d i v}} \mp \frac{\tau}{2}\left[\sum_{i=1}^{L} \frac{1}{2 m_{i}} \frac{\partial M_{k k l r(i)}^{(2,2) d i v}}{\partial x_{r}}+\sum_{i=1}^{L} \frac{1}{m_{i}} \frac{\partial M_{k l k r(i)}^{(2,2) d i v}}{\partial x_{r}}+\sum_{i=1}^{L} \frac{1}{2 m_{i}} \frac{\partial M_{k k l r(i)}^{(0,4)}}{\partial x_{r}}\right] \mp \\
& \mp \frac{\tau}{2}\left[\frac{1}{2} M_{k k r}^{(1,2)_{a p p}^{d i v}} \frac{\partial U_{l}}{\partial x_{r}}+\frac{1}{2} M_{l k r}^{(1,2) a p p} \frac{\partial U_{k}}{\partial x_{r}}+\frac{1}{2} M_{k l r}^{(1,2)_{a p p}^{d i v}} \frac{\partial U_{k}}{\partial x_{r}}\right]+ \\
& +\sum_{i=1}^{L} \frac{1}{2} M_{k k l(i)}^{(0,3)} a_{a p}^{d i v} \mp \frac{\tau}{2} \sum_{i=1}^{L} \frac{\partial}{\partial x_{r}}\left(\frac{1}{2} M_{l k k r(i)}^{(1,3) d i v}+M_{k k l r(i)}^{(1,3) d_{p p p}^{d i v}}\right)
\end{aligned}
$$

here,

$$
\begin{aligned}
q_{l}^{G}(t, \mathbf{x})=\sum_{i=1}^{L} q_{l(i)}^{G}(t, \mathbf{x}) & =\sum_{i, j=1}^{L} q_{l(i, j)}^{G}(t, \mathbf{x})=\sum_{i, j=1}^{L} \int m_{i}\left(G_{l}-U_{l}\right)(\mathbf{G}-\mathbf{U})^{2} f_{p(i, j)}\left(t, \mathbf{x}, \mathbf{G}, \mathbf{v}^{(i, j)}\right) d \mathbf{G} d \mathbf{v}^{(i, j)} \\
& q_{l}^{v}(t, \mathbf{x})=\sum_{i=1}^{L} q_{l(i)}^{v}(t, \mathbf{x})=\sum_{i, j=1}^{L} q_{l(i, j)}^{v}(t, \mathbf{x})= \\
& =\sum_{i, j=1}^{L} \int \frac{m_{i} m_{j}^{2}}{\left(m_{i}+m_{j}\right)^{2}}\left(G_{l}-U_{l}\right)\left(v^{(i, j)}\right)^{2} f_{p(i, j)}\left(t, \mathbf{x}, \mathbf{G}, \mathbf{v}^{(i, j)}\right) d \mathbf{G} d \mathbf{v}^{(i, j)} \\
& q_{l}^{G v a p p}(t, \mathbf{x})=\sum_{i=1}^{d i v} q_{l(i)}^{G v a p p}(t, \mathbf{x})=\sum_{i, j=1}^{L} q_{l(i, j)}^{G(d i v)}(t, \mathbf{x})= \\
& =\sum_{i, j=1}^{L} \int \frac{m_{i} m_{j}^{2}}{\left(m_{i}+m_{j}\right)^{2}}\left(G_{k}-U_{k}\right) v_{k}^{(i, j)} v_{l}^{(i, j)} f_{p(i, j)}\left(t, \mathbf{x}, \mathbf{G}, \mathbf{v}^{(i, j)}\right) d \mathbf{G} d \mathbf{v}^{(i, j)}
\end{aligned}
$$

Here, $\mathbf{q}=\mathbf{q}(t, \mathbf{x})$ is the heat flux. Value $\mathbf{q}_{d i v}^{G}=\mathbf{q}^{G}(t, \mathbf{x})$ allows for thermal energy transfer by moving centers of mass of particles; $\mathbf{q}^{v}=\mathbf{q}^{v}(t, \mathbf{x})$ and $\mathbf{q}^{G^{d}{ }^{d i v p}}=\mathbf{q}^{G^{d i p p}}(t, \mathbf{x})$ are responsible for thermal energy transfer due to relative motion of pair constituent particles. The fourth-order moments of pair distribution functions $M_{k l m n(i)}^{(2,2) d i v}=M_{k l m n(i)}^{(2,2)_{a p}^{d i v}}(t, \mathbf{x}), M_{k l m n}^{(0,4)}(i)=M_{k l m n}^{(0, i)}(i)$

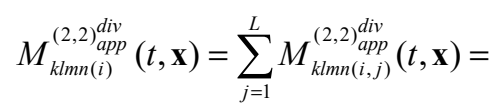

$$
\begin{aligned}
& =\sum_{j=1}^{L} \int \frac{m_{i}^{2} m_{j}^{2}}{\left(m_{i}+m_{j}\right)^{2}}\left(G_{k}-U_{k}\right)\left(G_{l}-U_{l}\right) v_{m}^{(i, j)} v_{n}^{(i, j)} f_{p(i, j)}^{d i v}\left(t, \mathbf{x}, \mathbf{G}, \mathbf{v}^{(i, j)}\right) d \mathbf{G} d \mathbf{v}^{(i, j)} \\
& M_{k l m n(i)}^{(0,4) d i v}(t, \mathbf{x})=\sum_{j=1}^{L} M_{k l m n(i, j)}^{(0,4) a_{p}^{d i v}}(t, \mathbf{x})=\sum_{j=1}^{L} \int \frac{m_{i}^{2} m_{j}{ }^{4}}{\left(m_{i}+m_{j}\right)^{4}} v_{k}^{(i, j)} v_{l}^{(i, j)} v_{m}^{(i, j)} v_{n}^{(i, j)} f_{p(i, j)}^{d i p}\left(t, \mathbf{x}, \mathbf{G}, \mathbf{v}^{(i, j)}\right) d \mathbf{G} d \mathbf{v}^{(i, j)} \\
& M_{k l m n(i)}^{(1,3)(t i v)}(t, \mathbf{x})=\sum_{j=1}^{L} M_{k l m n n}^{(1,3) d i, j)}(t, \mathbf{x})=
\end{aligned}
$$

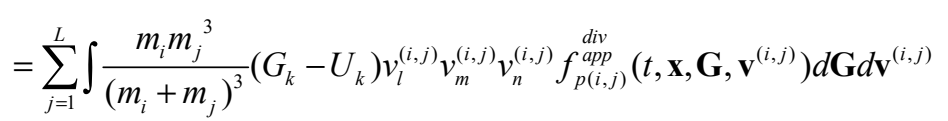

In the $G v$-moments of the third order (5.4), the first superscript pertains to the component of vector $\mathbf{G}$, while the second and third, to the component of vector $\mathbf{v}^{(i, j)}$. In the $G v$-moments of the fourth order (5.8), the first and second superscripts pertain to the components of vector $\mathbf{G}$, while the third and fourth, to the component of vector $\mathbf{v}^{(i, j)}$. Relationships (5.3)-(5.8) were derived with due regard for permutation property (A.3). 


\section{Grad Expansion}

Following Grad (1949), let us expend distribution function $f_{p(i, j)}^{d i v p}\left(t, \mathbf{x}, \mathbf{G}, \mathbf{v}^{(i, j)}\right)$ as a series, using a double set of Hermitian polynomials as a basis,

$$
\begin{aligned}
& f_{p(i, j)}^{d i v p}\left(t, \mathbf{x}, \mathbf{G}, \mathbf{v}^{(i, j)}\right)=n_{(i, j)}^{G}\left(\frac{m_{i}}{\pi k T^{G}}\right)^{\frac{3}{2}}\left(\frac{m_{i} m_{j}^{2}}{\left(m_{i}+m_{j}\right)^{2} \pi k T^{v}}\right)^{\frac{3}{2}} \exp \left[-\frac{m_{i}(\mathbf{G}-\mathbf{U})^{2}}{k T^{G}}\right] \times \\
& \times \exp \left[-\frac{m_{i} m_{j}^{2}\left(v^{(i, j)}\right)^{2}}{\left(m_{i}+m_{j}\right)^{2} k T^{v}}\right]\left[a_{(i, j)}^{(0,0)(i p p} Q^{0} H^{0}+\ldots+\frac{1}{k ! l !} a_{\alpha_{1} \ldots \alpha_{k} \beta_{1} \ldots \beta_{l}(i, j)}^{(k, l)} Q_{\alpha_{1} \ldots \alpha_{k}(i)}^{k} H_{\beta_{1} \ldots \beta_{l}(i, j)}^{l}\right]
\end{aligned}
$$

Here, $Q_{\alpha_{1} \ldots \alpha_{k}(i)}^{k}=Q_{\alpha_{1} \ldots \alpha_{k(i)}}^{k}\left(\mathbf{z}_{(i)}\right)$ and $H_{\beta_{1} \ldots \beta_{l}(i, j)}^{l}=H_{\beta_{1} \ldots \beta_{l}(i, j)}^{l}\left(\mathbf{y}_{(i, j)}\right)$ are the Hermitian polynomials whose properties were described, e.g., by Grad (1949); $\mathbf{z}_{(i)}=\left(2 m_{i} / k T^{G}\right)^{1 / 2}(\mathbf{G}-\mathbf{U}), \mathbf{y}_{(i, j)}=\left(2 m_{i} m_{j}^{2} /\left(m_{i}+m_{j}\right)^{2} k T^{v}\right)^{1 / 2} \mathbf{v}^{(i, j)}$. Expansion coefficients $a_{\alpha_{1} \ldots k_{k} \beta_{1} \ldots \beta_{l}(i, j)}^{(k, l) d i v}=a_{\alpha_{1} \ldots \alpha_{k} \beta_{1} \ldots \beta_{l}(i, j)}^{(k, d)}(t, \mathbf{x})$ are expressed in terms of hydrodynamic parameters using orthogonality of Hermitian polynomials,

$$
a_{\alpha_{1} \ldots \alpha_{k} \beta_{1} \ldots \beta_{l}(i, j)}^{(k, l d i v}(t, \mathbf{x})=\frac{1}{n_{(i, j)}^{G}} \int Q_{\alpha_{1} \ldots \alpha_{k}(i)}^{k} H_{\beta_{1} \ldots \beta_{l}(i, j)}^{l} f_{p(i, j)}^{d i v p}\left(t, \mathbf{x}, \mathbf{G}, \mathbf{v}^{(i, j)}\right) d \mathbf{G} d \mathbf{v}^{(i, j)}
$$

Let $\theta, \varphi$ and $v^{(i, j)}$ be the spherical coordinates of the vector $\mathbf{v}^{(i, j)}$. Let us next integrate Equation (6.1) respect to $\theta, \varphi$. In the resulting expansion, all addends involving $a_{\alpha_{1} \ldots \alpha_{k} \beta_{1} \ldots \beta_{l}(i, j)}^{(k, l)}$, but those with $k+l \leq 3$, are omitted. Furthermore, of all third-order addends $\quad(k+l=3)$, let us retain two terms proportional to $\mathbf{q}_{(i)}^{G}$ and $\mathbf{q}_{(i)}^{v}$, which, according to Equation (5.7), present the measured hydrodynamic value, thermal energy flux $\mathbf{q}$. Let us sum up the aforesaid expansion over $j$. Then, the resulting approximation is,

$$
\begin{gathered}
f_{p(i)}^{d i v}(t, \mathbf{x}, \mathbf{G}, v)=4 \pi n_{(i)}^{G}\left(\frac{m_{i}}{\pi k T^{G}}\right)^{\frac{3}{2}}\left(\frac{m_{i}}{4 \pi k T^{v}}\right)^{\frac{3}{2}} \exp \left[-\frac{m_{i}(\mathbf{G}-\mathbf{U})^{2}}{k T^{G}}\right] \exp \left[-\frac{m_{i} v^{2}}{4 k T^{v}}\right] \times \\
\times\left(a_{(i)}^{(0,0)}+a_{k(i)}^{(1,0)} Q_{k(i)}^{1}+\frac{1}{2} a_{k l(i)}^{(2,0)} Q_{k l(i)}^{2}+\frac{1}{6} a_{k(i)}^{(3,0)} Q_{k(i)}^{3}+\frac{1}{2} a_{k(i)}^{(1,2)} Q_{k(i)}^{1} H_{(i, i)}^{2}\right) .
\end{gathered}
$$

here,

$$
\begin{aligned}
f_{p(i, j)}^{d i v p}\left(t, \mathbf{x}, \mathbf{G}, v^{(i, j)}\right) & =\int f_{p(i, j)}^{d i v p}\left(t, \mathbf{x}, \mathbf{G}, \mathbf{v}^{(i, j)}\right) \sin \theta d \theta d \varphi \\
f_{p(i)}^{d i v} & (t, \mathbf{x}, \mathbf{G}, v)=\sum_{j=1}^{L} f_{p(i, j)}^{d i v p}\left(t, \mathbf{x}, \mathbf{G}, v^{(i, j)}\right) D_{[i, j]}
\end{aligned}
$$

The jakobian $D_{[i, j]}$ is specified in Equation (4.4). Coefficients appearing in Equation (6.3) are to be determined from Equation (6.2). After summation coefficients (6.3) become,

$$
\begin{gathered}
a_{(i)}^{(0,0)}=1, \quad a_{k(i)}^{(1,0)}=\left(\frac{2 m_{i}}{k T^{G}}\right)^{\frac{1}{2}}\left(U_{k(i)}^{G}-U_{k}\right), \quad a_{k l(i)}^{(2,0)}=\frac{p_{k l(i)}^{G}}{n_{(i)}^{G} k T^{G}}, \\
a_{k(i)}^{(3,0)}=\frac{3}{5}\left(\frac{2 m_{i}}{k T^{G}}\right)^{\frac{1}{2}}\left(\frac{2 q_{k(i)}^{G}}{n_{(i)}^{G} k T^{G}}-5 U_{k(i)}^{G}+5 U_{k}\right), \quad a_{k(i)}^{(1,2)}=\frac{1}{3}\left(\frac{2 m_{i}}{k T^{G}}\right)^{\frac{1}{2}}\left(\frac{2 q_{k(i)}^{v}}{n_{(i)}^{G} k T^{v}}-3 U_{k(i)}^{G}+3 U_{k}\right)
\end{gathered}
$$

Here, $Q_{k(i)}^{3}=Q_{k l(i)}^{3}$, and $H_{(i, i)}^{2}=H_{l l i, i)}^{2}$. All coefficients in Equation (6.4) can be expressed in terms of moments of the $f_{p(i)}(t, \mathbf{x}, \mathbf{G}, v)$ function, by which token superscripts div and app at them were omitted. Distribution function $f_{p(i, j)}^{d a p}\left(t, \mathbf{x}, \mathbf{G}, \mathbf{v}^{(i, j)}\right)$ can be recast as a sum of two terms,

$$
f_{p(i, j)}^{d i v}\left(t, \mathbf{x}, \mathbf{G}, \mathbf{v}^{(i, j)}\right)=\frac{1}{4 \pi} f_{p(i, j)}^{d i v}\left(t, \mathbf{x}, \mathbf{G}, v^{(i, j)}\right)+\Delta f_{p(i, j)}^{d i v}\left(t, \mathbf{x}, \mathbf{G}, \mathbf{v}^{(i, j)}\right)
$$




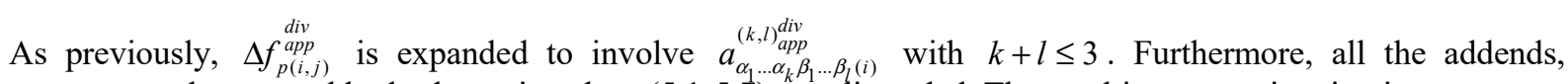
except ones that control hydrodynamic values (5.1-5.7), are discarded. The resulting approximation is,

$$
\begin{gathered}
\Delta f_{p(i, j)}^{\frac{d i v}{a p p}}\left(t, \mathbf{x}, \mathbf{G}, \mathbf{v}^{(i, j)}\right)=n_{(i, j)}^{G}\left(\frac{m_{i}}{\pi k T^{G}}\right)^{\frac{3}{2}}\left(\frac{m_{i} m_{j}^{2}}{\left(m_{i}+m_{j}\right)^{2} \pi k T^{v}}\right)^{\frac{3}{2}} \exp \left[-\frac{m_{i}(\mathbf{G}-\mathbf{U})^{2}}{k T^{G}}\right] \times \\
\times \exp \left[-\frac{m_{i} m_{j}^{2}\left(v^{(i, j)}\right)^{2}}{\left(m_{i}+m_{j}\right)^{2} k T^{v}}\right]\left(a_{k(i, j)}^{(0,1) d i v p} H_{k(i, j)}^{1}+\frac{1}{2} a_{k l(i, j)}^{(0,2) d_{a p p}^{d i v}} H_{k l(i, j)}^{2}+\frac{1}{2} a_{k(i, j)}^{(1,2) a p} Q_{l(i)}^{d i v}\left(H_{l k(i, j)}^{2}-\frac{1}{3} H_{(i, i)}^{2} \delta_{l k}\right)\right)
\end{gathered}
$$

Coefficients in Equation (6.6) equal,

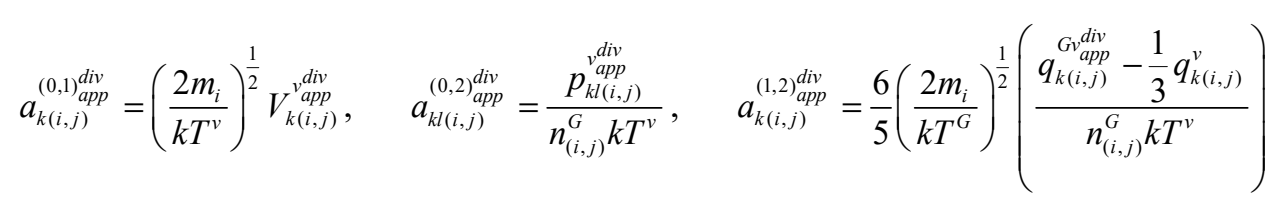

Let us consider the second-, third-, and fourth-order moments of pair distribution functions (5.2-5.8). These moments will be calculated using approximations (6.3) and (6.4) to $f_{p(i)}(t, \mathbf{x}, \mathbf{G}, v)$,

$$
\begin{array}{ccc}
p_{(i)}^{G}=n_{(i)}^{G} k T^{G} & p_{(i)}^{v}=n_{(i)}^{G} k T^{v} & M_{k l m(i)}^{(1,2)}=\frac{2}{3} q_{k(i)}^{v} \delta_{l m} \\
M_{k l m n(i)}^{(2,2)}=\frac{1}{4} P_{k l(i)}^{G} k T^{v} \delta_{m n} & M_{k l m n n}^{(0,4)}=\frac{1}{4} n_{(i)}^{G}\left(k T^{v}\right)^{2}\left(\delta_{k l} \delta_{m n}+\delta_{k m} \delta_{l n}+\delta_{k n} \delta_{l m}\right)
\end{array}
$$

Using approximation (6.6) and (6.7) to $\Delta f_{p(i, j)}^{d i v}\left(t, \mathbf{x}, \mathbf{G}, \mathbf{v}^{(i, j)}\right)$, let us specify the second-, third-, and fourth-order moments,

$$
\begin{aligned}
& \int \frac{m_{j}}{m_{i}+m_{j}} G_{l} v_{k} f_{p(i, j)}^{d i v}\left(t, \mathbf{x}, \mathbf{G}, \mathbf{v}^{(i, j)}\right) d \mathbf{G} d \mathbf{v}^{(i, j)}=n_{(i)}^{G} U_{l} V_{k(i)}^{v^{d i v p}} \\
& M_{k l r(i)}^{(0,3)}=n_{(i)}^{G}\left(V_{k(i)}^{v^{d i v p}} \delta_{l r}+V_{l(i)}^{d^{d a p p}} \delta_{k r}+V_{r(i)}^{d^{d a p p}} \delta_{k l}\right) \frac{k T^{v}}{2} \quad M_{k l m n(i)}^{(1,3)}=0
\end{aligned}
$$

In Equations (6.1-6.9), summation is done over repetitive subscripts. The accuracy of approximations (6.3) and (6.6) is discussed in what follows.

\section{Derivation of Hydrodynamics Equations}

Hydrodynamic equations will be derived from the Equation (4.28) for the $f_{1(i)}\left(t, \mathbf{x}, \boldsymbol{\xi}_{1}\right)$ one-particle distribution function. The basic property of the $f_{p(i)}(t, \mathbf{x}, \mathbf{G}, v)$ pair distribution function (4.15) will be used. Further, the formalism of the Grad method will not be invoked. More straightforward algorithm for deriving hydrodynamics equations will be used.

Let us integrate Equation (4.15) with respect to velocities and use weight function $\varphi_{\alpha_{1} \ldots \alpha_{p}}^{1(p)}(\mathbf{G}), \quad p=0,1,2$, and $\varphi^{2(q)}\left(m_{j}^{2}\left(v^{(i, j)}\right)^{2} /\left(m_{i}+m_{j}\right)^{2}\right), \quad q=0,1 \quad(4.16)$. Then, Equation (4.15) becomes,

$$
\begin{gathered}
\frac{\partial}{\partial t} \sum_{j=1}^{L} \int \varphi_{\alpha_{1} \ldots \alpha_{p}}^{(p, q)}\left(\mathbf{G},\left(v^{(i, j)}\right)^{2}\right) f_{p(i, j)}\left(t, \mathbf{x}, \mathbf{G}, v^{(i, j)}\right) d \mathbf{G}\left(v^{(i, j)}\right)^{2} d v^{(i, j)}+ \\
+\frac{\partial}{\partial x_{k}} \sum_{j=1}^{L} \int G_{k} \varphi_{\alpha_{1} \ldots \alpha_{p}}^{(p, q)}\left(\mathbf{G},\left(v^{(i, j)}\right)^{2}\right) f_{p(i, j)}\left(t, \mathbf{x}, \mathbf{G}, v^{(i, j)}\right) d \mathbf{G}\left(v^{(i, j)}\right)^{2} d v^{(i, j)}=0
\end{gathered}
$$

here,

$$
\tilde{\varphi}_{\alpha_{1} \ldots \alpha_{p}}^{(p, q)}\left(\mathbf{G}, v^{2}\right)=\varphi_{\alpha_{1} \ldots \alpha_{p}}^{(p, q)}\left(\mathbf{G},\left(v^{(i, j)}\right)^{2}\right)=\varphi_{\alpha_{1} \ldots \alpha_{p}}^{1(p)}(\mathbf{G}) \varphi^{2(q)}\left[\frac{m_{j}^{2}\left(v^{(i, j)}\right)^{2}}{\left(m_{i}+m_{j}\right)^{2}}\right]
$$

Let us integrate Equation (4.28) with respect to $\xi_{1}$. Let us subtract Equation (7.1) with $\varphi^{1(0)}=1, \varphi^{2(0)}=1$ from the aforesaid equation. Contribution of collision integral $J_{\alpha_{1} \ldots \alpha_{k}(i, j)}^{(k)}(t, \mathbf{x})$ is estimated in Appendix. With 
due regard for definitions of hydrodynamic values (5.1), (5.2), and (5.3), approximation (6.9), and collision integral $J_{(i)}^{(0)}(t, \mathbf{x})$ (A.11a), one obtains,

$$
\frac{\partial n_{(i)}^{G} V_{k(i)}^{v^{d i v}}}{\partial x_{k}} \mp \frac{\tau}{2} \frac{\partial^{2}}{\partial x_{k} \partial x_{l}}\left(\frac{1}{2 m_{i}} P_{k l(i)}^{v^{d a p}}\right) \mp \frac{\tau}{2} \frac{\partial^{2} n_{(i)}^{G} V_{k(i)}^{v^{d i v}}}{\partial t \partial x_{k}} \mp \frac{\tau}{2} \frac{\partial^{2} n_{(i)}^{G} U_{k} V_{l(i)}^{v^{d i v p}}}{\partial x_{k} \partial x_{l}}=0
$$

Let us multiply Equation (4.28) by $m_{i} \xi_{1 k}$, integrate it with respect to $\xi_{1}$, and sum up it over $i$. Let us sum up Equation (7.1) with $m_{i} \varphi_{k}^{1(1)}=m_{i} G_{k}, \varphi^{2(0)}=1$ over $i$ and subtract the result from the aforesaid equation. Using definitions (5.2)-(5.7), approximations (6.8) and (6.9), and collision integral $J_{k}^{(1)}(t, \mathbf{x})$ (A.11b), one obtains,

$$
\left.\frac{\partial}{\partial x_{l}}\left(\frac{1}{2} P_{k l}^{v^{d} a p p}\right) \mp \frac{\tau}{2} \frac{\partial^{2}}{\partial x_{l} \partial x_{r}}\left[\frac{1}{3} q_{k}^{v} \delta_{l r}+\frac{1}{3} q_{l}^{v} \delta_{k r}+\frac{1}{2} U_{k} P_{l r}^{P^{d a p}}+\frac{1}{2} U_{l} P_{k r}^{P_{k r p}^{d i v}}\right)\right] \mp \frac{\tau}{2} \frac{\partial^{2}}{\partial t \partial x_{l}}\left(\frac{1}{2} P_{k l}^{v^{d a p p}}\right)=0
$$

Let us multiply Equation (4.28) by $m_{i} \xi_{1}^{2} / 2$, integrate it with respect to $\xi_{1}$, and sum up it over $i$. In Equation (7.1), let the weight function be successively $m_{i} \varphi^{1(2)} / 2=m_{i} G^{2} / 2, \quad \varphi^{2(0)}=1$, and $\varphi^{1(0)}=1$, $m_{i} \varphi^{2(1)} / 2=m_{i} m_{j}^{2}\left(v^{(i, j)}\right)^{2} / 2\left(m_{i}+m_{j}\right)^{2}$. Let us sum up Equation (7.1) over $i$ and subtract the results from the aforesaid equation. Using definitions (5.2)-(5.7), approximations (6.8) and (6.9), and collision integral $J^{(2)}(t, \mathbf{x})$ (A.11c), one obtains,

$$
\begin{aligned}
& \frac{\partial}{\partial x_{l}}\left(q_{l}^{{ }_{l}^{G}{ }^{d i v p}}+\frac{1}{2} U_{k} P_{k l}^{v_{a p p}^{d i v}}+\frac{5}{4} k T^{v} \sum_{i=1}^{L} n_{(i)}^{G} V_{l(i)}^{v^{d i v p}}\right) \mp \frac{\tau}{2} \frac{\partial^{2}}{\partial x_{l} \partial x_{k}} \sum_{i=1}^{L}\left[\frac{5 p_{(i)}^{v}\left(k T^{G}+k T^{v}\right) \delta_{k l}}{8 m_{i}}+\frac{p_{k l(i)}^{G} k T^{v}}{4 m_{i}}\right] \mp \\
& \mp \frac{\tau}{2} \frac{\partial^{2}}{\partial x_{l} \partial x_{k}}\left[\frac{1}{3} U_{r} q_{r}^{v} \delta_{l k}+\frac{1}{3} U_{r} q_{k}^{v} \delta_{l r}+\frac{1}{3} U_{k} q_{r}^{v} \delta_{l r}+\frac{1}{4} U^{2} p^{v} \delta_{k l}+\frac{1}{2} U_{k} U_{l} p^{v}\right] \mp \\
& \mp \frac{\tau}{2} \frac{\partial^{2}}{\partial t \partial x_{l}}\left(q_{l}^{G{ }^{d v i v p}}+\frac{1}{2} U_{k} P_{k l}^{v^{d i v p}}+\frac{5}{4} k T^{v} \sum_{i=1}^{L} n_{(i)}^{G} V_{l(i)}^{d^{d a p p}}\right)=0
\end{aligned}
$$

Let us multiply Equation (4.28) by $\xi_{1 k}$ and integrate it with respect to $\xi_{1}$. Let us subtract Equation (7.1) with $\varphi_{k}^{1(1)}=G_{k}, \varphi^{2(0)}=1$ from the aforesaid equation. Using definitions (5.1),(5.2), and (5.3), approximation (6.9), and collision integrals $J_{k(i)}^{(1)}(t, \mathbf{x})$ (A.12) and $J_{k l(i)}^{(2)}(t, \mathbf{x})$ (A.15), one obtains,

$$
\frac{1}{2 m_{i}} \frac{\partial p_{(i)}^{v}}{\partial x_{k}}+B_{k(i)}^{a p p}=-\frac{1}{\tau} n_{(i)}^{G} V_{k(i)}^{v a p p}
$$

Let us multiply Equation (4.28) by $m_{i} \xi_{1 k} \xi_{1 l}$, integrate it with respect to $\xi_{1}$, and sum over $i$. In Equation (7.1), let the weight function be successively $m_{i} \varphi_{k l}^{1(2)}=m_{i} G_{k} G_{l}, \quad \varphi^{2(0)}=1$ and $\varphi^{1(0)}=1$, $m_{i} \varphi^{2(1)} / 3=m_{i} m_{j}^{2}\left(v^{(i, j)}\right)^{2} / 3\left(m_{i}+m_{j}\right)^{2}$. Let us sum up Equation (7.1) over $i$ and subtract the results from the aforesaid equation. Using definitions (5.2)-(5.7), approximations (6.8) and (6.9), and collision integral $J_{k l}^{(2)}(t, \mathbf{x})$ (A.13), one obtains,

$$
\frac{\partial}{\partial x_{r}}\left(\frac{1}{3} q_{l}^{v} \delta_{k r}+\frac{1}{3} q_{k}^{v} \delta_{l r}+\frac{1}{2} U_{k} p^{v} \delta_{l r}+\frac{1}{2} U_{l} p^{v} \delta_{k r}\right)+B_{k l}^{a p p}=-\frac{1}{2 \tau} p_{k l}^{v a p p}
$$

Let us multiply Equation (4.28) by $m_{i} \xi_{1 k} \xi_{1}^{2} / 2$, integrate it with respect to $\xi_{1}$, and sum up it over $i$. In Equation (7.1), let the weight function be successively $m_{i} \varphi_{k}^{1(3)} / 2=m_{i} G_{k} G^{2} / 2, \varphi^{2(0)}=1$ and $\varphi_{k}^{1(1)}=G_{k}$, $5 m_{i} \varphi^{2(1)} / 6=5 m_{i} m_{j}^{2}\left(v^{(i, j)}\right)^{2} / 6\left(m_{i}+m_{j}\right)^{2}$. Let us sum up Equation (7.1) over $i$ and subtract the results from the aforesaid equation. Using definitions (5.2)-(5.7), approximations (6.8) and (6.9), and collision integral $J_{k}^{(3)}(t, \mathbf{x})$ (A.14), one obtains,

$$
\begin{gathered}
\frac{\partial}{\partial x_{l}} \sum_{i=1}^{L}\left[\frac{5 p_{(i)}^{v}\left(k T^{G}+k T^{v}\right) \delta_{k l}}{8 m_{i}}+\frac{p_{k l(i)}^{G} k T^{v}}{4 m_{i}}\right]+ \\
+\frac{1}{3}\left(q_{k}^{v} \delta_{r l}+q_{l}^{v} \delta_{k r}+q_{r}^{v} \delta_{l k}\right) \frac{\partial U_{r}}{\partial x_{l}}+B_{k}^{a p p}=-\frac{1}{\tau}\left(q_{k}^{G v a p p}-\frac{1}{3} q_{k}^{v}\right)-\frac{5 k T^{v}}{4 \tau} \sum_{i=1}^{L} n_{(i)}^{G} V_{k(i)}^{v a p p}
\end{gathered}
$$


On left hand side of Equations (7.5)-(7.7), all terms proportional to $\tau$ explicitly have been discarded. Let us multiply Equation (4.9) successively by $v_{k}^{(i, i)} / 2, \quad m_{i}\left(v_{k}^{(i, i)} v_{l}^{(i, i)}-\left(v^{(i, i)}\right)^{2} \delta_{k l} / 3\right) / 4$, and $m_{i} G_{l}\left(v_{k}^{(i, i)} v_{l}^{(i, i)}-\left(v^{(i, i)}\right)^{2} \delta_{k l} / 3\right) / 4+m_{i}\left(v^{(i, i)}\right)^{2} v_{k}^{(i, i)} / 16$, and make use relationship (2.6). Integrate the resulting equations with respect to $\mathbf{G}$ and $\mathbf{v}^{(i, j)}$, and sum up it over $i$. Using definitions (5.2)-(5.7), symmetry property (A.9), and integrals (A.10), one obtains,

$$
\begin{gathered}
B_{k(i)}^{d i v}=-\frac{1}{\tau} n_{(i)}^{G} V_{k(i)}^{v d i v} \\
B_{k l}^{d i v}=-\frac{1}{2 \tau} p_{k l}^{v d i v} \\
B_{k}^{d i v}=-\frac{1}{\tau}\left(q_{k}^{G v d i v}-\frac{1}{3} q_{k}^{v}\right)-\frac{5 k T^{v}}{4 \tau} \sum_{i=1}^{L} n_{(i)}^{G} V_{k(i)}^{v d i v}
\end{gathered}
$$

Generally, Equations (7.5)-(7.7) and (7.8)-(7.10) look like,

$$
\begin{aligned}
& A_{k(i)}+B_{k(i)}^{d i v p}=-\frac{1}{\tau} n_{(i)}^{G} V_{k(i)}^{v_{a p p}^{d i v}} \\
& A_{k l}+B_{k l}^{\stackrel{d i v}{a p p}}=-\frac{1}{2 \tau} p_{k l}^{\stackrel{d a p p}{d i v}} \\
& A_{k}+B_{k}^{d i v p}=-\frac{1}{\tau}\left(q_{k}^{G v_{a p p}^{d i v}}-\frac{1}{3} q_{k}^{v}\right)-\frac{5 k T^{v}}{4 \tau} \sum_{i=1}^{L} n_{(i)}^{G} V_{k(i)}^{v_{a p p}^{d i v}}
\end{aligned}
$$

Here, $A_{k(i)}=A_{k(i)}(t, \mathbf{x}), A_{k l}=A_{k l}(t, \mathbf{x})$, and $A_{k}=A_{k}(t, \mathbf{x})$ involve only the moments of the $f_{p(i)}(t, \mathbf{x}, \mathbf{G}, v)$ function (Equations (6.3) and (6.4)) undergoing, as follows from (4.15), no changes in collisions. Functions $A_{k(i)}, A_{k l i v}$, and $A_{k}$ do not depend on $\tau$ explicitly. Functions $B_{k(i)}^{d i v}=B_{k(i)}^{d i v}(t, \mathbf{x}), \quad B_{k l}^{d i p p}=B_{k l}^{d i v p}(t, \mathbf{x})$, and $B_{k}^{a p p}=B_{k}^{a p p}(\underset{d i v}{a, \mathbf{x}})$ involve exclusively the moments of the $\Delta_{d i v}^{\substack{d i v \\ \text { div }}}(t, \mathbf{x}, \mathbf{G}, \mathbf{v})$ function (Equations (6.6) and (6.7)). Functions $\begin{gathered}d i v \\ B_{k(i)}^{a p p}\end{gathered}, B_{k l}^{d i v}$, and $B_{k}^{a p p}$ do not depend on $\tau$ explicitly. In the hydrodynamic stage of description, the characteristic space and time scales of variation of hydrodynamic values far exceed the characteristic free path length $\lambda$ and intercollision time $\tau$. Assuming that all terms except those linear in $\tau$ can be discarded in hydrodynamics stage, Equations (7.5)-(7.7), and (7.8)-(7.10) can be specified as,

$$
\begin{gathered}
V_{k(i)}^{v a p p}=-\tau \frac{p^{v}}{2 m_{i} n_{(i)}^{G}} d_{k}^{(i)} \\
V_{k(i)}^{v d i v}=0
\end{gathered}
$$

here,

$$
\begin{gathered}
\frac{p^{v}}{m_{i} n_{(i)}^{G}} d_{k}^{(i)}=\frac{1}{m_{i} n_{(i)}^{G}} \frac{\partial p_{(i)}^{v}}{\partial x_{k}}-\frac{1}{\rho^{G}} \frac{\partial p^{v}}{\partial x_{k}} \\
p_{k l}^{v a p p}=-\tau p^{v}\left(\frac{\partial U_{k}}{\partial x_{l}}+\frac{\partial U_{l}}{\partial x_{k}}-\frac{2}{3} \delta_{l k} \frac{\partial U_{r}}{\partial x_{r}}\right)-\frac{2}{3} \tau\left(\frac{\partial q_{k}^{v}}{\partial x_{l}}+\frac{\partial q_{l}^{v}}{\partial x_{k}}-\frac{2}{3} \delta_{l k} \frac{\partial q_{r}^{v}}{\partial x_{r}}\right) \\
p_{k l}^{v d v}=0 \\
q_{k}^{\text {Gvapp }}-\frac{1}{3} q_{k}^{v}=-\tau \sum_{i=1}^{L}\left[\frac{5 p_{(i)}^{v}}{8 m_{i}} \frac{\partial\left(k T^{G}+k T^{v}\right)}{\partial x_{k}}+\frac{1}{4 m_{i}} \frac{\partial k T^{v} p_{k l(i)}^{G}}{\partial x_{l}}+\frac{5 p^{v} k T^{G}}{8 m_{i}} d_{k}^{(i)}\right]-
\end{gathered}
$$




$$
\begin{gathered}
-\frac{\tau}{3}\left(q_{k}^{v} \delta_{r l}+q_{l}^{v} \delta_{k r}+q_{r}^{v} \delta_{l k}\right) \frac{\partial U_{r}}{\partial x_{l}} \\
q_{k}^{\text {Gvdiv }}-\frac{1}{3} q_{k}^{v}=0
\end{gathered}
$$

Divergence-free form (7.15) of stress tensor $p_{k l}^{\text {vapp }}$ is derived from Equation (7.18). Equation (7.17) gives the possibility to introduce the diffusional thermodynamic force $d_{k}^{(i)}$ on the right hand sides of both Equation (7.14) for diffusion velocity $V_{k(i)}^{v a p p}$ and Equation (7.16) for heat flux $\left(q_{k}^{\text {Gvapp }}-q_{k}^{v} / 3\right)$. In Equations (7.3) and (7.4), let us discard all terms linear in $\tau$, then,

$$
\begin{gathered}
\frac{\partial p^{v}}{\partial x_{k}}[1+O(K n)]=0 \\
\frac{1}{3} \frac{\partial q_{k}^{v}}{\partial x_{k}}+\frac{1}{2} p^{v} \frac{\partial U_{k}}{\partial x_{k}}[1+O(K n)]=0
\end{gathered}
$$

Here, $K n=\lambda / L_{h}$.

\section{Discussion}

The lower properties of a separate particle of $i$-quality (its mass $m_{i}$, momentum $m_{i} \xi_{1}$, and energy $m_{i} \xi_{1}^{2} / 2$ ) are invariants of the Boltzmann collision integral. The moments of a one-particle distribution function constructed on these invariants determine $L+2$ principle hydrodynamic values, namely, the number density $i$-quality particles $n_{(i)}(t, \mathbf{x}), i=1, \ldots, L$, and the velocity and temperature of the medium, $\mathbf{U}(t, \mathbf{x})$ and $T(t, \mathbf{x})$, respectively. The multiplication of the Boltzmann collision integral by any of these invariants results in the disappearance of the collision integral (Ferziger \& Kaper, 1972). This means that binary interparticle collisions do not influence the distribution of the principle hydrodynamic values. These distributions are determined by the initial and boundary conditions of the problem. All the other higher properties of a separate particle are not invariants of the Boltzmann collision integral. For this reason, binary interparticle collisions have a determining influence on the distributions of hydrodynamic values constructed on the higher properties. They tune the distributions of these hydrodynamic values to the distributions of the principle hydrodynamic values (Ferziger \& Kaper, 1972).

The velocity of the center of mass $\mathbf{G}$ and the energy of relative motion $m_{i} v^{2} / 4$ are binary particle collision invariants. For this reason, the collision integral (4.9) is invariant not only with respect to $\mathbf{G}$ and $m_{i} v^{2} / 4$ but also with respect to any property of two particles $\tilde{\varphi}_{\alpha_{1} \ldots \alpha_{p}}^{(p, q)}\left(\mathbf{G}, v^{2}\right), l=0,1, \ldots, q=0,1$, that is, a function of these values. The $\tilde{\varphi}_{\alpha_{1} \ldots \alpha_{p}}^{(p, q)}\left(\mathbf{G}, v^{2}\right)$ property is therefore a collision invariant. It follows that all the moments of the $f_{p(i)}(t, \mathbf{x}, \mathbf{G}, v)$ function $(4.15)$ constructed on the $\tilde{\varphi}_{\alpha_{1} \ldots \alpha_{p}}^{(p, q)}\left(\mathbf{G}, v^{2}\right)$ property are principle hydrodynamic values. As in classic hydrodynamics, binary collisions tune the distributions of hydrodynamic values constructed on properties other than $\tilde{\varphi}_{\alpha_{1} \ldots \alpha_{p}}^{(p, q)}\left(\mathbf{G}, v^{2}\right)$ to the distributions of the principle hydrodynamic values. An increase in the number of principal hydrodynamic values taken into account will cause the additional appearance of derivatives of all the principal moments taken into account in both definitions and equations.

The principle hydrodynamic values $n_{(i)}^{G}, \mathbf{U}_{(i)}^{G}, p^{G}, p^{v}, p_{k l(i)}^{G}, q_{k}^{G}$, and $q_{k}^{v}$ are given by Equations (5.1)-(5.7). Let us express the measured moments of one particle distribution function in terms of the principle hydrodynamic values. To do this, let us invoke Equations (7.2)-(7.4) and relationships (7.14)-(7.16) relating non-principle hydrodynamic values to principle hydrodynamic values. The zero-order moments assume the form:

$$
n_{(i)}=n_{(i)}^{G} \quad n=n^{G} \quad \rho=\rho^{G}
$$

The first-order moments assume the form:

$$
\begin{aligned}
\mathbf{U}=\mathbf{U}^{G} & =\frac{1}{\rho} \sum_{i=1}^{L} m_{i} n_{(i)} \mathbf{U}_{(i)}^{G} \quad \mathbf{U}_{(i)}=\mathbf{U}+\mathbf{V}_{(i)} \quad \mathbf{V}_{(i)}=\mathbf{V}_{(i)}^{G}+\mathbf{V}_{(i)}^{v} \\
\mathbf{V}_{(i)}^{G} & =\mathbf{U}_{(i)}^{G}-\mathbf{U} \quad \sum_{i=1}^{L} m_{i} n_{(i)} \mathbf{V}_{(i)}^{v}=0 \quad \sum_{i=1}^{L} m_{i} n_{(i)} \mathbf{V}_{(i)}^{G}=0
\end{aligned}
$$

here, 


$$
\begin{array}{cc}
V_{k(i)}^{v}=-\sum_{j=1}^{L} D_{i j} d_{k}^{(j)} & d_{k}^{(i)}=\frac{\partial}{\partial x_{k}}\left(\frac{n_{(i)}}{n}\right)+\left(\frac{n_{(i)}}{n}-\frac{m_{i} n_{(i)}}{\rho}\right) \frac{\partial \ln p^{v}}{\partial x_{k}} \\
D_{i j}=\frac{p^{v} \tau}{4 \rho}\left(\frac{\rho}{m_{j} n_{(j)}} \delta_{i j}-1\right) & \sum_{i=1}^{L} m_{i} n_{(i)} D_{i j}=0
\end{array}
$$

In Equation (7.14), diffusional thermodynamic forces $d_{k}^{(i)}, i=1, \ldots, L$, are not linearly independent. Following Ferziger \& Kaper (1972), let us switch from vectors $d_{k}^{(i)}$ to vectors $d_{k}^{*(i)}$,

$$
d_{k}^{(i)}=d_{k}^{*(i)}-\frac{m_{i} n_{(i)}}{\rho} \sum_{j=1}^{L} d_{k}^{*(j)}
$$

With due regard for linear dependence (8.2b), one returns to the thermodynamic forces $d_{k}^{(i)}$. The second-order moments assume the form:

$$
\begin{gathered}
p=\frac{1}{2} p^{G}+\frac{1}{2} p^{v} \quad k T=\frac{1}{2} k T^{G}+\frac{1}{2} k T^{v} \\
P_{k l}=\frac{1}{2} P_{k l}^{G}+\frac{1}{2} P_{k l}^{v} \quad p_{k l}=\frac{1}{2} p_{k l}^{G}+\frac{1}{2} p_{k l}^{v}
\end{gathered}
$$

here,

$$
p_{k l}^{v}=-\frac{1}{2} \tau p^{v}\left(\frac{\partial U_{k}}{\partial x_{l}}+\frac{\partial U_{l}}{\partial x_{k}}-\frac{2}{3} \delta_{l k} \frac{\partial U_{r}}{\partial x_{r}}\right)-\frac{1}{3} \tau\left(\frac{\partial q_{k}^{v}}{\partial x_{l}}+\frac{\partial q_{l}^{v}}{\partial x_{k}}-\frac{2}{3} \delta_{l k} \frac{\partial q_{r}^{v}}{\partial x_{r}}\right)
$$

The third-order moments assume the form:

$$
q_{k}=\frac{1}{2} q_{k}^{G}+\frac{5}{6} q_{k}^{v}+\left(q_{k}^{G v}-\frac{1}{3} q_{k}^{v}\right)
$$

here,

$$
\begin{gathered}
\left(q_{k}^{G v}-\frac{1}{3} q_{k}^{v}\right)=-\frac{p^{v} \tau}{2} \sum_{i=1}^{L}\left[\frac{5 n_{(i)} k}{4 n m_{i}} \frac{\partial T}{\partial x_{k}}\right]+\sum_{i=1}^{L} \frac{5 n_{(i)} k T}{2} V_{k(i)}^{v}- \\
-\frac{\tau}{2} \sum\left[\frac{1}{4 m_{i}} \frac{\partial k T^{v} p_{k l(i)}^{G}}{\partial x_{l}}\right]-\frac{\tau}{6}\left(q_{k}^{v} \delta_{r l}+q_{l}^{v} \delta_{k r}+q_{r}^{v} \delta_{l k}\right) \frac{\partial U_{r}}{\partial x_{l}}
\end{gathered}
$$

The lower one-particle distribution function moments $n_{(i)}, n, \mathbf{U}$, and $p$ are written through the principal hydrodynamic values $n_{(i)}^{G}, n^{G}, \mathbf{U}_{(i)}^{G}, p^{G}$, and $p^{v}$ only. The measurable moments $\mathbf{V}_{(i)}, p$, and $\mathbf{q}$ are represented by linear combinations of principal and non-principal hydrodynamic values. The $\mathbf{V}_{(i)}^{k}$ diffusion velocity component is a principal hydrodynamic value. The $\mathbf{V}_{(i)}^{v}$ diffusion velocity component is the linear combination of the vectors of diffusional thermodynamic forces. The $p_{k l}^{G}$ stress tensor component is a principal hydrodynamic value. The first term of the $p_{k l}^{v}$ component is proportional to the tensor of velocities of deformations $U_{k l}$ (the Newton law), the remaining term of the $p_{k l}^{v}$ component appears because of an increase in the number of principal hydrodynamic values. The first two components of the heat flux vector, $\mathbf{q}^{G}$ and $\mathbf{q}^{v}$, are principal hydrodynamic values. The first term of the third component $\left(\mathbf{q}^{G v}-\mathbf{q}^{v} / 3\right)$ is proportional to the temperature gradient (the Fourier law), the second term of the third component $\left(\mathbf{q}^{G v}-\mathbf{q}^{v} / 3\right)$ corresponds to the enthalpy flux due to diffusion of particles, and the other terms of the third component appear because of an increase in the number of principal hydrodynamic values.

The ultimate set of hydrodynamic equations involves an interminable chain of "linked" equations and Equations (7.2)-(7.4). All "linked" equations of the chain are represented by Equation (7.1). The lower equations of the chain assume the form: 


$$
\begin{gathered}
\frac{\partial n_{(i)}}{\partial t}+\frac{\partial n_{(i)}\left(U_{k}+V_{k(i)}^{G}\right)}{\partial x_{k}}=0 \\
\frac{\partial \rho}{\partial t}+\frac{\partial \rho U_{k}}{\partial x_{k}}=0 \\
\frac{\partial \rho U_{k}}{\partial t}+\frac{\partial}{\partial x_{l}}\left(\frac{1}{2} P_{k l}^{G}+\rho U_{k} U_{l}\right)=0 \\
\frac{\partial}{\partial t}\left[\frac{3}{4} n k T^{G}+\frac{1}{2} \rho U^{2}\right]+\frac{\partial}{\partial x_{k}}\left[\frac{1}{2} q_{k}^{G}+\frac{3}{4} n U_{k} k T^{G}+\frac{1}{2} U_{l} P_{k l}^{G}+\frac{1}{2} \rho U^{2} U_{k}\right]=0 \\
\frac{\partial}{\partial t}\left[\frac{3}{4} n k T^{v}\right]+\frac{\partial}{\partial x_{k}}\left[\frac{1}{2} q_{k}^{v}+\frac{3}{4} n U_{k} k T^{v}\right]=0
\end{gathered}
$$

With due regard for Equations (7.14)-(7.16), Equations (7.2)-(7.4) assume the form,

$$
\begin{gathered}
\frac{\partial}{\partial x_{k}}\left(-n_{(i)} \sum_{j=1}^{L} D_{i j} d_{k}^{(j)}\right)=0 \\
\frac{\partial}{\partial x_{l}}\left(\frac{1}{2} p^{v} \delta_{k l}+\frac{1}{2} p_{k l}^{v}\right)=0 \\
\frac{\partial}{\partial x_{k}}\left[\frac{1}{3} q_{k}^{v}+\frac{1}{2} U_{k} p^{v}+\left(q_{k}^{G v}-\frac{1}{3} q_{k}^{v}\right)+\frac{1}{2} U_{l} p_{k l}^{v}\right]=0
\end{gathered}
$$

The general structure of the Equations (8.6)-(8.9) and (8.11)-(8.12) is identical to the structure of appropriate multimoment conservation laws in a gas of identical particles. The equation (8.10) appears because of divergence of the diffusional flux. Taking into account definitions (8.1)-(8.4), it is easily verified that chain equation (8.5), as added to Equation (8.10) yields the equation of conservation of number of particles of $i$ - quality. This conservation equation, when summarized over $i$, yields the discontinuity condition (8.6). Chain equation (8.7), as added to Equation (8.11), yields the equation of conservation of momentum, while chain equations (8.8) and (8.9) in combination with Equation (8.12) yield the equation of conservation of energy. Thus, each of the multimoment conservation laws is divided into the separate equations. The first group of conservation equations (8.5), (8.7), (8.8), and (8.9) describes the convective phenomena. The second group of conservation equations (8.10)-(8.12) describes the dissipative phenomena. None of the terms in Equations (8.10)-(8.12) are labeled by div or app superscripts. Thus, the resulting hydrodynamics equations are independent of whether the pair distribution functions, in terms of which they are expressed, correspond to approaching or diverging particles.

The classic hydrodynamics recognizes $L+2$ principal hydrodynamic values for gas mixture, $n_{(i)}, i=1, \ldots, L$, $\mathbf{U}$, and $T$. Multimoment hydrodynamics equations are constructed using $3 L+4$ principal hydrodynamic values, $n_{(i)}, \mathbf{U}_{(i)}^{G}, p_{k l(i)}^{G}, i=1, \ldots, L, \quad T^{G}, T^{v}, \mathbf{q}^{G}$, and $\mathbf{q}^{v}$. Fundamental property of the $f_{p(i)}(t, \mathbf{x}, \mathbf{G}, v)$ pair distribution function (4.15) reveals the existence of an infinite number of principle hydrodynamic values (4.16). Only $3 L+4$ principal hydrodynamic values were retained in Grad expansion (6.3). Just these values specify measurable one-particle distribution function moments. The other principle hydrodynamic values (6.8) were calculated using approximation (6.3)-(6.4). Only $3 L-1$ non-principal hydrodynamic values were retained in Grad expansion (6.6), $\mathbf{V}_{(i)}^{v}, p_{k l(i)}^{v}, \quad\left(\mathbf{q}_{(i)}^{G v}-\mathbf{q}_{(i)}^{v} / 3\right)$. Just these values specify measurable one-particle distribution function moments. The other non-principle hydrodynamic values (6.9) were calculated using approximation (6.6)-(6.7).

The characteristic free path $\lambda_{i, j}$ is introduced at the stage of the formation of pair distribution functions (3.1). The characteristic time between collisions $\tau$ is contained in resulting kinetic equations (4.9), (4.10) and (4.14) and hydrodynamics equations (8.10)-(8.12). However, present formalism of deriving the hydrodynamics equations is not intended to produce expression for indeterminate parameter $\tau$. As previously (Lebed, 1996b), the coefficient $\tau$ may be expressed in terms of transport coefficients (Ferziger \& Kaper, 1972).

An analysis of multimoment hydrodynamics equations in the limit of a weak deviation of the system state from the state of statistical equilibrium was performed by Lebed (1996b) in a gas of identical structureless particles. 
The analysis showed that multimoment hydrodynamics equations and classic three-moment hydrodynamics equations become identical only in the limit of a weak deviation of the system state from the state of the statistical equilibrium. It was, in particular, shown by Lebed (1996b) that, in the $\operatorname{Re} \ll 1$ limit,

$$
p=p^{G}=p^{v} \quad p_{k l}=p_{k l}^{G}=p_{k l}^{v}
$$

Moreover, the second term on the right-hand side of Equation (8.3b) responsible for viscous stresses, which appears because of heat fluxes, becomes negligibly small in the $\mathrm{Re} \ll 1$ limit compared with the first term. It follows that, according to Lebed (1996b), the $p_{k l}$ viscous stress tensor becomes directly proportional to the tensor of velocities of deformations $U_{k l}$ in the $\mathrm{Re} \ll 1$ limit. According to Equation (8.3b), the $\tau p^{v} / 2$ value containing an indefinite parameter $\tau$ is a proportionality factor between $p_{k l}$ and $U_{k l}$. The identical relation between $p_{k l}$ and $U_{k l}$ is contained in classic hydrodynamics equations derived directly from the Boltzmann kinetic equation. Moreover, classic kinetic theory allows the proportionality factor between $p_{k l}$ and $U_{k l}$ known as the dynamic shear viscosity coefficient $\tilde{\eta}$ to be calculated (Ferziger \& Kaper, 1972). The analysis by Lebed (1996b) remains true for a gas mixture. By setting the coefficients of identical gradients of hydrodynamic values equal to each other, one obtains the equation for the $\tau$ parameter in the form,

$$
\tau_{s}=\frac{2 \tilde{\eta}}{p^{v}}
$$

The coefficient $\tilde{\eta}$ can be calculated with arbitrary accuracy by solving the Boltzmann equation with the use of the Chapman-Enscog method for an arbitrary law of interparticle interactions (Ferziger \& Kaper, 1972).

To find therelation between parameter $\tau$ and other transport coefficients, it is necessary to perform analysis of Equations (8.5)-(8.12) in the limit of a weak deviation of the system state from the state of statistical equilibrium. However, there is another way of approximation. Following Ferziger \& Kaper (1972), let us write a formal expression analogous Eucken ratio,

$$
\frac{\tilde{\lambda}}{\tilde{\eta}}=\frac{15 k}{4 \bar{m}}, \quad \sum_{i=1}^{L} \frac{n_{(i)}}{m_{i}}=\frac{n}{\bar{m}}
$$

Here, $\tilde{\lambda}$ is the heat conductivity coefficient. With due regard for Equations (8.14), one obtains,

$$
\tau_{h}=\frac{8 \bar{m} \tilde{\lambda}}{15 k p^{v}}
$$

The coefficient $\tilde{\lambda}$ can be calculated with arbitrary accuracy by solving the Boltzmann equation (Ferziger \& Kaper, 1972). Approximations made when calculating the collision integral (A.8) give only one independent diffusion coefficient $D_{i j}, i \neq j$. That is, all the off-diagonal diffusion coefficients are equal to each other,

$$
-D_{i j}=\tilde{D}=\frac{p^{v} \tau_{d}}{4 \rho} \quad i \neq j
$$

With due regard for Equations (8.16), one obtains,

$$
\tau_{d}=\frac{4 \rho \tilde{D}}{p^{v}}
$$

The coefficient $\tilde{D}$ can be calculated with arbitrary accuracy by solving the Boltzmann equation. Present formalism of deriving the hydrodynamics equations is not capable of reproducing the thermal diffusion phenomenon (Ferziger \& Kaper, 1972).

Different meanings of the intercollision time $\tau$ should be used for interpretation of different transfer properties. To interpret viscous stress phenomena, Equation (8.14) for $\tau_{s}$ should be substitute in Equation (8.3b). To interpret heat conductivity phenomena, Equation (8.15) for $\tau_{h}$ should be substitute in Equation (8.4b). To interpret diffusion phenomena, Equation (8.17) for $\tau_{d}$ should be substitute in Equation (8.2b).

Collision integral (4.28) is calculated in Appendix. Collision integral (4.28) was estimated for a gas particles modeled by rigid spheres. It turned out that this approximation greatly facilitates estimation of collision integral (4.28). In this case, in integration in velocity with some weight function of velocity, collision integral is expressed exactly in terms of the moments of pair distribution functions. The classic Boltzmann collision integral offers similar advantages to the case of the so-called Maxwellian molecules (Ferziger \& Kaper, 1972). 
Both the neglect of particles forming no pair and use of common intercollision time $\tau$ are the approximations of the same order. In Lebed (1996b), all these effects were called "terminal". As demonstrated by Lebed (1996a), neglect of "terminal" effects does not distort gas modeling in terms one-particle distribution function even in kinetic stage. It exerts even lesser effect on the accuracy of hydrodynamic modeling.

The structure of hydrodynamic equations is known to be independent of the form of the law of structureless particle interaction. The interaction law only influences the values of transport coefficients (Ferziger \& Kaper, 1972). It follows that derived multimoment hydrodynamics equations are valid for the description of gas flows with an arbitrary law of interaction of structureless particles. Equations (8.10)-(8.12) were reduced to involve the terms linear in $\tau$. At the hydrodynamic stage of modeling, omitted nonlinear terms correspond to the Barnett accuracy (Ferziger \& Kaper, 1972). It follows that multimoment hydrodynamics equations presented above correspond to Navier-Stokes accuracy of description.

\section{References}

Ferziger, J. H., \& Kaper, H. G. (1972). Mathematical theory of transport processes in gases. Amsterdam: North-Holland Publishing Company.

Grad, H. (1949). About kinetic theory of rarefied gases. Communications on Pure and Applied Mathematics, 2(4), 331-407.

Lebed, I. V. (1990). Equations of pair distribution functions. Chemical Physics Letters, 165(1-2), 226-228. http://dx.doi.org/10.1016/0009-2614(90)85433-D

Lebed, I. V. (1995). Derivation of the equations for pair distribution functions. Chemical Physics Reports, 14(5), 599-615.

Lebed, I. V. (1996a). Method of two-particle distribution functions. Hydrodynamic equations. Chemical Physics Reports, 15(6), 861-883.

Lebed, I. V. (1996b). Hydrodynamic equations stemming from two-particle distributions in the limit of weak non-equilibrium. Analysis of invertibility of equations. Chemical Physics Reports, 15(12), 1725- 1750.

Lebed, I. V. (1997). The method of pair functions as applied to the problem of a flow around a quiescent solid sphere. Chemical Physics Reports, 16(7), 1263-1301.

Lebed, I. V. (1998). About the behavior of the entropy of a gas flow losing its stability. Chemical Physics Reports, 17(3), 411-439.

Lebed, I. V., \& Umanskii, S. Y. (2007). The appearance and development of turbulence in a flow past a sphere: problems and the existing approaches to their solution. Russian Journal of Physical Chemistry B, 1(1), 52-73.http://dx.doi.org/10.1134/S1990793107010071

Lebed, I. V., \& Umanskii, S. Y. (2012). On the possibility of improving classic hydrodynamics equations by an increase in the number of hydrodynamic values. Russian Journal of Physical Chemistry B, 6(1), 149-162.http://dx.doi.org/10.1134/S1990793112010204

Lebed, I. V. (2013). About the prospects for passage to instability. Open Journal of Fluid Dynamics, 3(3), 214-229.http://dx.doi.org/10.4236/ojfd.2013.33027

Lebed, I. V. (2014a). Development of instability in the problem of flow around a sphere. Russian Journal of Physical Chemistry B, 8(2), 240-253.http://dx.doi.org/10.1134/S1990793114020171

Lebed, I. V. (2014b). Multimoment hydrodynamics in problem on flow around a sphere: entropy interpretation of the appearance and development of instability. Open Journal of Fluid Dynamics, 4(2), 163-206.http://dx.doi.org/10.4236/ojfd.2014.42015

Lebed, I. V. (2014c). About appearance of the irreversibility. Open Journal of Fluid Dynamics, 4(3), 298-320.http://dx.doi.org/10.4236/ojfd.2014.43023

Lebed, I. V. (2015a). Kinetic foundation for the multimoment hydrodynamics equations. Open Journal of Fluid Dynamics, 5(1), 76-91. http://dx.doi.org/10.4236/ojfd.2015.51010

Lebed, I. V. (2015b). Evolution of unstable system. Journal of Advances in Physics, 9(3), 2487-2502.

Liboff, R.L. (1969). Introduction to the theory of kinetic equations. New York -London-Sydney-Toronto: John Willey\&Sons.

Patterson, G. N. (1956). Molecular flow of gases. New York: John Willey\&Sons. 


\section{Appendix}

Let us specify the permutation property,

$$
F_{2(i, j)}^{p( \pm)}\left(t, \mathbf{x}_{1}, \xi_{1}, \mathbf{x}_{2}, \xi_{2}\right)=F_{2(j, i)}^{p( \pm)}\left(t, \mathbf{x}_{2}, \xi_{2}, \mathbf{x}_{1}, \xi_{1}\right)
$$

In accordance with Equation (A.1), the probability that at some time $t$ one particle of $i$-quality finds itself within an unit element of phase space near point $\mathbf{x}_{1}, \boldsymbol{\xi}_{1}$, and another particle of $j$-quality, within an unit element near point $\mathbf{x}_{2}, \boldsymbol{\xi}_{2}$, equals the probability that at some time $t$ one particle of $j$-quality finds itself within an unit element of phase space near point $\mathbf{x}_{2}, \boldsymbol{\xi}_{2}$, and another particle of $i$-quality, within an unit element near point $\mathbf{x}_{1}, \boldsymbol{\xi}_{1}$.

Let us recast Equation (A.1) first in terms of the $\tilde{\tilde{F}}_{2(i, j)}^{p( \pm)}\left(t, \mathbf{x}_{1}, \boldsymbol{\xi}_{1}, \mathbf{v}^{(i, j)}, \boldsymbol{\rho}^{(i, j)}\right)$ function (2.4), then in terms of the $\tilde{F}_{2(i, j)}^{p(t)}\left(t, \mathbf{x}, \mathbf{G}, \mathbf{v}^{(i, j)}, \mathbf{\rho}^{(i, j)}\right)$ function (2.4), and at last in terms of the $\breve{F}_{2(i, j)}^{p( \pm)}\left(t, \breve{\mathbf{x}}_{1}^{(i, j)}, \breve{\boldsymbol{\xi}}_{1}^{(i, j)}, \breve{\mathbf{v}}^{(i, j)}, \breve{\boldsymbol{\rho}}^{(i, j)}\right)$ function (2.13),

$$
\begin{gathered}
\tilde{\tilde{F}}_{2(i, j)}^{p( \pm)}\left(t, \mathbf{x}_{1}, \boldsymbol{\xi}_{1}, \mathbf{v}^{(i, j)}, \boldsymbol{\rho}^{(i, j)}\right)=\tilde{\tilde{F}}_{2(j, i)}^{p(t)}\left(t, \mathbf{x}_{2}^{(i, j)}, \xi_{2}^{(i, j)},-\mathbf{v}^{(i, j)},-\mathbf{\rho}^{(i, j)}\right) \\
\tilde{F}_{2(i, j)}^{p( \pm)}\left(t, \mathbf{x}, \mathbf{G}, \mathbf{v}^{(i, j)}, \boldsymbol{\rho}^{(i, j)}\right)=\tilde{F}_{2(j, i)}^{p( \pm)}\left(t, \mathbf{x}, \mathbf{G},-\mathbf{v}^{(i, j)},-\mathbf{\rho}^{(i, j)}\right) \\
\breve{F}_{2(i, j)}^{p( \pm)}\left(t, \breve{\mathbf{x}}_{1}^{(i, j)}, \breve{\xi}_{1}^{(i, j)}, \breve{\mathbf{v}}^{(i, j)}, \breve{\boldsymbol{\rho}}^{(i, j)}\right)=\breve{F}_{2(j, i)}^{p( \pm)}\left(t, \breve{\mathbf{x}}_{2}^{(i, j)}, \breve{\xi}_{2}^{(i, j)},-\breve{\mathbf{v}}^{(i, j)},-\breve{\boldsymbol{\rho}}^{(i, j)}\right)
\end{gathered}
$$

Let us recast the permutation property (A.2) in terms of the $F_{p(j, i)}^{\substack{a i p \\ a p}}\left(t, \mathbf{x}, \mathbf{G}, \mathbf{v}^{(i, j)}\right)$ functions (3.1). The next step will be multiply aforesaid property by some weight function of velocity $\mathbf{v}^{(i, j)}$ and mass. Integrating with respect to $\mathbf{v}^{(i, j)}$ and summation over $i, j$ yield,

$$
\begin{gathered}
\sum_{i, j=1}^{L} \int \frac{m_{i} m_{j}}{m_{i}+m_{j}} \mathbf{v}^{(i, j)} F_{p(i, j)}^{\text {adv }}\left(t, \mathbf{x}, \mathbf{G}, \mathbf{v}^{(i, j)}\right) d \mathbf{v}^{(i, j)}=0 \\
\sum_{i, j=1}^{L} \int \frac{m_{i} m_{j}^{2}}{\left(m_{i}+m_{j}\right)^{2}} v_{k}^{(i, j)} v_{l}^{(i, j)} F_{p(i, j)}^{d i v}\left(t, \mathbf{x}, \mathbf{G}, \mathbf{v}^{(i, j)}\right) d \mathbf{v}^{(i, j)}= \\
=\sum_{i, j=1}^{L} \int \frac{m_{j} m_{i}^{2}}{\left(m_{i}+m_{j}\right)^{2}} v_{k}^{(i, j)} v_{l}^{(i, j)} F_{p(i, j)}^{d i v p}\left(t, \mathbf{x}, \mathbf{G}, \mathbf{v}^{(i, j)}\right) d \mathbf{v}^{(i, j)} \\
\sum_{i, j=1}^{L} \int \frac{m_{i} m_{j}^{3}}{\left(m_{i}+m_{j}\right)^{3}} v_{k}^{(i, j)}\left(v^{(i, j)}\right)^{2} F_{p(i, j)}^{d i v p}\left(t, \mathbf{x}, \mathbf{G}, \mathbf{v}^{(i, j)}\right) d \mathbf{v}^{(i, j)}= \\
=-\sum_{i, j=1}^{L} \int \frac{m_{j} m_{i}^{3}}{\left(m_{i}+m_{j}\right)^{3}} v_{k}^{(i, j)}\left(v^{(i, j)}\right)^{2} F_{p(i, j)}^{d i v p}\left(t, \mathbf{x}, \mathbf{G}, \mathbf{v}^{(i, j)}\right) d \mathbf{v}^{(i, j)}
\end{gathered}
$$

Let us consider collision integral $J_{(i, j)}\left(t, \mathbf{x}, \boldsymbol{\xi}_{1}\right)$, Equation (4.28). Symmetry properties of the $J_{(i, j)}\left(t, \mathbf{x}, \boldsymbol{\xi}_{1}\right)$ collision integral correspond to symmetry properties of the Boltzmann collision integral (Ferziger \& Kaper, 1972). Suppose that

$$
\begin{gathered}
J_{\alpha_{1} \ldots p_{k}(i, j)}^{(k) a p}(t, \mathbf{x})=\int \varphi_{\alpha_{1} \ldots \alpha_{k}}^{(k)}\left(\xi_{1}, m_{i}\right) J_{(i, j)}\left(t, \mathbf{x}, \xi_{1}\right) d \xi_{1}= \\
=\iint_{0}^{2 \pi} \int_{0}^{d_{i, j}} \varphi_{\alpha_{1} \ldots \alpha_{k}}^{(k)}\left(\xi_{1}, m_{i}\right) v^{(i, j)} N_{i} \tilde{N}_{j}\left[\overline{\bar{F}}_{2(i, j)}^{p(-)}\left(t, \mathbf{x}, \xi_{1}^{(i, j)}, \mathbf{v}^{(i, j)},-d_{i, j}\right)-\right.
\end{gathered}
$$




$$
\left.-\overline{\bar{F}}_{2(i, j)}^{p(-)}\left(t, \mathbf{x}, \boldsymbol{\xi}_{1}, \mathbf{v}^{(i, j)},-d_{i, j}\right)\right] \frac{b d b d \varepsilon}{\sigma_{i, j}} d \mathbf{v}^{(i, j)} d \boldsymbol{\xi}_{1}
$$

Here, $\varphi_{\alpha_{1} \ldots \alpha_{k}}^{(k)}\left(\xi_{1}, m_{i}\right)$ is some weight function of velocity $\xi_{1}$. By virtue of symmetry of integral $J_{(i, j)}\left(t, \mathbf{x}, \boldsymbol{\xi}_{1}\right)$,

$$
\begin{aligned}
& J_{\alpha_{1} \ldots \alpha_{k}(i, j)}^{(k) a p p}(t, \mathbf{x})=-\iint_{0}^{2 \pi} \int_{0}^{2 \pi} \varphi_{\alpha_{1} \ldots \alpha_{k}}^{(k)}\left(\xi_{1}^{(i, j)}, m_{i}\right) v^{(i, j)} N_{i} \tilde{N}_{j} \times \\
& \times\left[\overline{\bar{F}}_{2(i, j)}^{p(-)}\left(t, \mathbf{x}, \xi_{1}^{(i, j)}, \mathbf{v}^{(i, j)},-d_{i, j}\right)-\overline{\bar{F}}_{2(i, j)}^{p(-)}\left(t, \mathbf{x}, \boldsymbol{\xi}_{1}, \mathbf{v}^{(i, j)},-d_{i, j}\right)\right] \frac{b d b d \varepsilon}{\sigma_{i, j}} d \mathbf{v}^{(i, j)} d \xi_{1}= \\
& =\iint_{0}^{2 \pi} \int_{0}^{d_{i, j}}\left[\varphi_{\alpha_{1} \ldots \alpha_{k}}^{(k)}\left(\xi_{1}^{(i, j)}, m_{i}\right)-\varphi_{\alpha_{1} \ldots \alpha_{k}}^{(k)}\left(\boldsymbol{\xi}_{1}, m_{i}\right)\right] v^{(i, j)} N_{i} \tilde{N}_{j} \overline{\bar{F}}_{2(i, j)}^{p(-)}\left(t, \mathbf{x}, \boldsymbol{\xi}_{1}, \mathbf{v}^{(i, j)},-d_{i, j}\right) \frac{b d b d \varepsilon}{\sigma_{i, j}} d \mathbf{v}^{(i, j)} d \boldsymbol{\xi}_{1}
\end{aligned}
$$

Collision integral $J_{(i, j)}\left(t, \mathbf{x}, \xi_{1}\right)$ has no symmetry with respect to subscripts $i$ and $j$. However, summation over $i$ and $j$ eliminates this non-symmetry, $N_{i} \tilde{N}_{j}=N_{j} \tilde{N}_{i}$,

$$
\begin{gathered}
\sum_{i, j=1}^{L} \iint_{0}^{2 \pi} \int_{0}^{d_{i, j}} \varphi_{\alpha_{1} \ldots \alpha_{k}}^{(k)}\left(\boldsymbol{\xi}_{1}, m_{i}\right) v^{(i, j)} N_{i} \tilde{N}_{j}\left[\overline{\bar{F}}_{2(i, j)}^{p(-)}\left(t, \mathbf{x}, \boldsymbol{\xi}_{1}^{(i, j)}, \mathbf{v}^{(i, j)},-d_{i, j}\right)-\right. \\
\left.-\overline{\bar{F}}_{2(i, j)}^{p(-)}\left(t, \mathbf{x}, \boldsymbol{\xi}_{1}, \mathbf{v}^{(i, j)},-d_{i, j}\right)\right] \frac{b d b d \varepsilon}{\sigma_{i, j}} d \mathbf{v}^{(i, j)} d \boldsymbol{\xi}_{1}= \\
=\sum_{i, j=1}^{L} \int_{0}^{2 \pi} \int_{0}^{d_{i, j}} \varphi_{\alpha_{1} \ldots \alpha_{k}}^{(k)}\left(\xi_{2}^{(i, j)}, m_{j}\right) v^{(i, j)} N_{j} \tilde{N}_{i}\left[\overline{\bar{F}}_{2(j, i)}^{p(-)}\left(t, \mathbf{x}, \xi_{2}^{(i, j)},-\mathbf{v}^{(i, j)},-d_{i, j}\right)-\right. \\
\left.-\overline{\bar{F}}_{2(j, i)}^{p(-)}\left(t, \mathbf{x}, \boldsymbol{\xi}_{2}^{(i, j)},-\mathbf{v}^{(i, j)},-d_{i, j}\right)\right] \frac{b d b d \varepsilon}{\sigma_{i, j}} d \mathbf{v}^{(i, j)} d \xi_{1}
\end{gathered}
$$

Invoking symmetry property (A.7), permutation property (A.2), and relationship (2.14), one obtains,

$$
\begin{gathered}
J_{\alpha_{1} \ldots \alpha_{k}}^{(k) a p p}(t, \mathbf{x})=\frac{1}{2} \sum_{i, j=1}^{L} \iint_{0}^{2 \pi} \int_{0}^{d_{i, j}}\left[\varphi_{\alpha_{1} \ldots \alpha_{k}}^{(k)}\left(\xi_{2}^{(i, j)}, m_{j}\right)+\varphi_{\alpha_{1} \ldots \alpha_{k}}^{(k)}\left(\xi_{1}^{(i, j)}, m_{i}\right)-\right. \\
\left.-\varphi_{\alpha_{1} \ldots \alpha_{k}}^{(k)}\left(\xi_{2}^{(i, j)}, m_{j}\right)-\varphi_{\alpha_{1} \ldots \alpha_{k}}^{(k)}\left(\xi_{1}, m_{i}\right)\right] v^{(i, j)} N_{i} \tilde{N}_{j} \overline{\tilde{F}}_{2(i, j)}^{p(-)}\left(t, \mathbf{x}, \xi_{1}, \mathbf{v}^{(i, j)},-d_{i, j}\right) \frac{b d b d \varepsilon}{\sigma_{i, j}} d \mathbf{v}^{(i, j)} d \xi_{1} \\
J_{\alpha_{1} \ldots \alpha_{k}}^{(k) a p}(t, \mathbf{x})=\sum_{i=1}^{L} J_{\alpha_{1} \ldots \alpha_{k}(i)}^{(k) a p p}(t, \mathbf{x})=\sum_{i, j=1}^{L} J_{\alpha_{1} \ldots \alpha_{k}(i, j)}^{(k) a p}(t, \mathbf{x})
\end{gathered}
$$

Let us consider collision integral (4.9). This collision integral possesses the following symmetry property,

$$
\begin{aligned}
& \iint_{0}^{1} \int_{0}^{1} \varphi_{\alpha_{1} \ldots \alpha_{k}}^{(k)}\left(\mathbf{G}, \mathbf{v}^{(i, j)}, m_{i}\right) f_{p(i, j)}^{d i v p}\left(t, \mathbf{x}, \mathbf{G}, \mathbf{v}^{(i, j)}\right) \tilde{b} d \tilde{b} d \tilde{\varepsilon} d \mathbf{G} d \mathbf{v}^{(i, j)}= \\
& =\iint_{0}^{1} \int_{0}^{1} \varphi_{\alpha_{1} \ldots \alpha_{k}}^{(k)}\left(\mathbf{G}, \mathbf{v}^{(i, j)}, m_{i}\right) f_{p(i, j)}^{d i v}\left(t, \mathbf{x}, \mathbf{G}, \mathbf{v}^{(i, j)}\right) \tilde{b} d \tilde{b} d \tilde{\varepsilon} d \mathbf{G} d \mathbf{v}^{(i, j)}
\end{aligned}
$$

Here, $b=d_{i, j} \hat{b}, \varepsilon=2 \pi \hat{\varepsilon}$. Let us restrict ourselves to the case of solid spherical particles with radiuses $d_{i}$ and $d_{j}, d_{i, j}=d_{i}+d_{j}$. From the dynamic theory of binary collision of solid spheres (Patterson, 1956), one obtains,

$$
\int_{0}^{2 \pi} \int_{0}^{d_{i, j}} v_{k}^{(i, j)} b d b d \mathcal{E}=0 \int_{0}^{2 \pi} \int_{0}^{d_{i, j}} v_{k}^{(i, j)} v_{l}^{\prime(i, j)} b d b d \mathcal{E}=\frac{1}{3}\left(v^{(i, j)}\right)^{2} \sigma_{i, j} \delta_{k l}
$$

Let us consider collision integrals $J_{\alpha_{1} \ldots \alpha_{k}(i)}^{(k) a p p}(t, \mathbf{x})$ (A.6) and $J_{\alpha_{1} \ldots \alpha_{k}}^{(k) a p p}(t, \mathbf{x}) \quad$ (A.8). Collision invariants $1, m_{i} \xi_{1}$, and $m_{i} \xi_{1}^{2} / 2$ will be successively chosen as weight function $\varphi_{\alpha_{1} \ldots \alpha_{k}}^{\left(\alpha_{1}\right) \ldots \alpha_{k}}\left(\xi_{1}, m_{i}\right)$. Then, in accordance with conservation laws, one obtains, 


$$
\begin{array}{cc}
J_{(i)}^{(0) a p p}(t, \mathbf{x})=0 & \varphi^{(0)}\left(\boldsymbol{\xi}_{1}, m_{i}\right)=1 \\
J_{k}^{(1) a p p}(t, \mathbf{x})=0 & \varphi_{k}^{(1)}\left(\xi_{1}, m_{i}\right)=m_{i} \xi_{1 k} \\
J^{(2) a p p}(t, \mathbf{x})=0 & \varphi^{(2)}\left(\xi_{1}, m_{i}\right)=m_{i} \xi_{1}^{2} / 2
\end{array}
$$

The next step will be multiply collision integral $J_{(i)}\left(t, \mathbf{x}, \xi_{1}\right)$ (4.24) by $\xi_{1 k}$ and integrate it with respect to $\xi_{1}$. Let us use symmetry property (A.6). Invoking relationships (3.12) and (3.5), let us recast the aforesaid integral first in terms of the $\tilde{F}_{2(i, j)}^{p(-)}\left(t, \mathbf{x}, \mathbf{G}, \mathbf{v}^{(i, j)},-d_{i, j}\right)$ function, and then in terms of the $f_{p(i, j)}^{a p p}\left(t, \mathbf{x}, \mathbf{G}, \mathbf{v}^{(i, j)}\right)$ function. In view of Equation (A.10), collision integral $J_{(i)}^{(1) a p p}(t, \mathbf{x})$ with $\varphi_{k}^{(1)}\left(\boldsymbol{\xi}_{1}, m_{i}\right) / m_{i}=\xi_{1 k}$ assumes the form,

$$
J_{k(i)}^{(1) a p p}(t, \mathbf{x})=-\frac{1}{\tau} n_{(i)}^{G} V_{k(i)}^{v a p p}+\tilde{B}_{k(i)}^{a p p}
$$

The last step will be multiply collision integral $J_{(i)}\left(t, \mathbf{x}, \xi_{1}\right)(4.28)$ successively by $m_{i} \xi_{1 k} \xi_{1 l}$ and $m_{i} \xi_{1 l} \xi_{1}^{2} / 2$, integrate it with respect to $\xi_{1}$, and sum up it over $i$. Make use representation (A.8) and switch from velocities $\boldsymbol{\xi}_{1}, \mathbf{v}^{(i, j)}$ to velocities $\mathbf{G}, \mathbf{v}^{(i, j)}$. Invoking relationship (3.12), let us switch from the $\tilde{\tilde{F}}_{2(i, j)}^{p(-)}\left(t, \mathbf{x}, \boldsymbol{\xi}_{1}, \mathbf{v}^{(i, j)},-d_{i, j}\right)$ function to the $\tilde{\tilde{F}}_{2(i, j)}^{p(-)}\left(t, \mathbf{x}, \mathbf{G}, \mathbf{v}^{(i, j)},-d_{i, j}\right)$ function. With due regard for equation (A.10), one obtains,

$$
\begin{gathered}
J_{k l}^{(2) a p p}(t, \mathbf{x})=\sum_{i, j=1}^{L} \int \frac{1}{2}\left(\frac{m_{i} m_{j}^{2}+m_{i}^{2} m_{j}}{\left(m_{i}+m_{j}\right)^{2}}\right)\left(\frac{1}{3}\left(v^{(i, j)}\right)^{2} \delta_{k l}-v_{k}^{(i, j)} v_{l}^{(i, j)}\right) \times \\
\times v^{(i, j)} N_{i} \tilde{N}_{j} \overline{\tilde{F}}_{2(i, j)}^{p(-)}\left(t, \mathbf{x}, \mathbf{G}, \mathbf{v}^{(i, j)},-d_{i, j}\right) d \mathbf{G} d \mathbf{v}^{(i, j)} \\
J_{k}^{(3) a p p}(t, \mathbf{x})=\sum_{i, j=1}^{L} \int\left[\frac{1}{2}\left(\frac{m_{i} m_{j}^{2}+m_{i}^{2} m_{j}}{\left(m_{i}+m_{j}\right)^{2}}\right) G_{l}\left(\frac{1}{3}\left(v^{(i, j)}\right)^{2} \delta_{k l}-v_{k}^{(i, j)} v_{l}^{(i, j)}\right)-\right. \\
\left.-\frac{1}{4}\left(\frac{m_{i} m_{j}^{3}-m_{i}^{3} m_{j}}{\left(m_{i}+m_{j}\right)^{3}}\right)\left(v^{(i, j)}\right)^{2} v_{k}^{(i, j)}\right] v^{(i, j)} N_{i} \tilde{N}_{j} \overline{\tilde{F}}_{2(i, j)}^{p(-)}\left(t, \mathbf{x}, \mathbf{G}, \mathbf{v}^{(i, j)},-d_{i, j}\right) d \mathbf{G} d \mathbf{v}^{(i, j)}
\end{gathered}
$$

Let us use the permutation properties (A.4a) and (A.4b). With due regard for relationship (3.5), let us recast the aforesaid collision integrals in terms of the $f_{p(i)}^{a p p}\left(t, \mathbf{x}, \mathbf{G}, \mathbf{v}^{(i, j)}\right)$ function and invoke approximation (6.9). Then, collision integrals $J_{k l}^{(2) a p p}(t, \mathbf{x})$ with $\varphi_{k l}^{(2)}\left(\xi_{1}, m_{i}\right)=m_{i} \xi_{1 k} \xi_{1 l}$, and $J_{l}^{(3) a p p}(t, \mathbf{x})$ with $\varphi_{l}^{(3)}\left(\xi_{1}, m_{i}\right)=m_{i} \xi_{1 l} \xi_{1}^{2} / 2$ assume the form,

$$
\begin{gathered}
J_{k l}^{(2) a p p}(t, \mathbf{x})=\sum_{i=1}^{L} J_{k l(i)}^{(2) a p p}(t, \mathbf{x})=-\frac{1}{2 \tau} p_{k l}^{\text {vapp }}+\tilde{B}_{k l}^{\text {app }} \\
J_{l}^{(3) a p p}(t, \mathbf{x})=-\frac{1}{\tau}\left(q_{l}^{\text {Gvapp }}-\frac{1}{3} q_{l}^{v}\right)-\frac{5 k T^{v}}{4 \tau} \sum_{i=1}^{L} n_{i} V_{l(i)}^{v a p p}-\frac{1}{2 \tau} U_{k} p_{k l}^{\text {vapp }}+\tilde{B}_{k}^{\text {app }}
\end{gathered}
$$

In Equation (A13),

$$
J_{k l(i)}^{(2) a p p}(t, \mathbf{x})=-\frac{1}{\tau} m_{i} n_{(i)}^{G} U_{l} V_{k(i)}^{v a p p}-\frac{1}{\tau} m_{i} n_{(i)}^{G} U_{k} V_{l(i)}^{v a p p}-\frac{1}{2 \tau} p_{k l(i)}^{v a p p}+\tilde{B}_{k l(i)}^{a p p}
$$

Functions $\tilde{B}_{k(i)}^{a p p}=\tilde{B}_{k(i)}^{a p p}(t, \mathbf{x}), \quad \tilde{B}_{k l}^{a p p}=\tilde{B}_{k l}^{a p p}(t, \mathbf{x}), \quad \tilde{B}_{k l(i)}^{a p p}=\tilde{B}_{k l(i)}^{a p p}(t, \mathbf{x})$, and $\tilde{B}_{k}^{a p p}=\tilde{B}_{k}^{a p p}(t, \mathbf{x})$ involve exclusively the moments of the $\Delta f_{p(i)}^{a p p}\left(t, \mathbf{x}, \mathbf{G}, \mathbf{v}^{(i, j)}\right)$ function (Equations (6.6), and (6.7)). Functions $\tilde{B}_{k(i)}^{a p p}, \tilde{B}_{k l}^{a p p}, \tilde{B}_{k l(i)}^{a p p}$, and $\tilde{B}_{k}^{\text {app }}$ do not depend on $\tau$ explicitly.

\section{Copyrights}

Copyright for this article is retained by the author(s), with first publication rights granted to the journal.

This is an open-access article distributed under the terms and conditions of the Creative Commons Attribution license (http://creativecommons.org/licenses/by/4.0/). 